\title{
Scoring methods for joint damage on plain radiographs in rheumatoid arthritis : progressive understanding of methodological issues
}

Citation for published version (APA):

Bruynesteyn, K. (2004). Scoring methods for joint damage on plain radiographs in rheumatoid arthritis : progressive understanding of methodological issues. [Doctoral Thesis, Maastricht University]. Datawyse / Universitaire Pers Maastricht. https://doi.org/10.26481/dis.20040617kb

Document status and date:

Published: 01/01/2004

DOI:

10.26481/dis.20040617kb

Document Version:

Publisher's PDF, also known as Version of record

Please check the document version of this publication:

- A submitted manuscript is the version of the article upon submission and before peer-review. There can be important differences between the submitted version and the official published version of record.

People interested in the research are advised to contact the author for the final version of the publication, or visit the DOI to the publisher's website.

- The final author version and the galley proof are versions of the publication after peer review.

- The final published version features the final layout of the paper including the volume, issue and page numbers.

Link to publication

\footnotetext{
General rights rights.

- You may freely distribute the URL identifying the publication in the public portal. please follow below link for the End User Agreement:

www.umlib.nl/taverne-license

Take down policy

If you believe that this document breaches copyright please contact us at:

repository@maastrichtuniversity.nl

providing details and we will investigate your claim.
}

Copyright and moral rights for the publications made accessible in the public portal are retained by the authors and/or other copyright owners and it is a condition of accessing publications that users recognise and abide by the legal requirements associated with these

- Users may download and print one copy of any publication from the public portal for the purpose of private study or research.

- You may not further distribute the material or use it for any profit-making activity or commercial gain

If the publication is distributed under the terms of Article 25fa of the Dutch Copyright Act, indicated by the "Taverne" license above, 


\section{Scoring methods for joint damage on plain radiographs in rheumatoid arthritis \\ Progressive understanding of methodological issues}


Q Bruynesteyn Karin, Maastrich 2004

ISBN 9052784124

Production: Datawyse | Universitaire Pers Maastricht

The publications of this thesis was financially supported by Abbot B.V., Bio-Imaging Technologies Inc, Dutch Arthritis Association, NV Organon, Schering..Plough B.V., Wyeth Pharmaceuticals. 


\title{
Scoring methods for joint damage on plain radiographs in rheumatoid arthritis Progressive understanding of methodological issues
}

\author{
PROEFSCHRIFT \\ ter verkrijging wan de graad van doctor \\ aan de Universiteit Maastricht, \\ op gezag van de Rector Magnificus, Prof. Mr. G.P.M.F. Mols, \\ volgens het besluit wan het College van Decanen, \\ in het openbaar te verdedigen \\ op donderdag 17 juni 2004 am 16.00 uur
}

door

Karin Bruynesteym

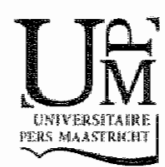




\section{Promotores}

Prof. dr. D.M.F.M. van der Heijde

Prof. dr. J.M.J.P. wan der Linder

\section{Beoordelingscommissie}

Prof. dr. C.P. van Schayck (woorzitter)

Prof. dr. M. Boers (Vrije Universiteit Amsterdam)

Prof. dr. J.M.A. van Engelshoven

Prof. dr. J.T. Sharp (University of Washington, USA)

Prof. dr. C.P.M. van der Vleuten 
Woor min ouders 



\section{Contents}

Chopter 1 Introduction.

Chapter 2 The Sharp/van der Heijde method aut-pertomed the Larsen/Scott method on the individual patient level in assessing radiographs in early rheurnatoid arthritis.

Chapier 3 Contribution of progression of erosive damage in previously eroded joints in early rheumatoid anthritis trials: COBRA trials as an example.

Chapter 4 Determination of the Minimal Clinically Important Difference in rheumatoid arthritis joint damage of the Sharp/van der Heijde and Larsen/Scott scoring methods by clinical expents and comparison with the Smallesi Detectable Difference.

Chapter 5 Progression of theumatoid arthritis on plain radiagraphs judged differently by expert radiologists and theumatologists.

Chapter 6 Detecting radiological changes in rheumaloid arthritis that are considered important by experts: influence of reading with or without known sequence.

Chopter 7 Deciding on progression of joint damage in paired films of individual patients: smallest detectable difference or change?

Chapter 8 How the type of risk reduction influences required sample sizes in randomized clinical irials.

Chapter 9 Radiography as primary outcome in rheumatoid anthritis: acceptable sample sizes for trials with 3 months follow-up.

Chapter 10 General discussion and conclusions.

Chapter 11 Summary.

Samenvatting.

Dankwoord.

About the author. 

Chapter 1

Introduction 
Chopter I 


\section{Introduction}

\section{Background thesis}

\section{The disease rheumatoid arthritis}

Rheumatoid arthritis (RA) is a chronic and systemic inflammatory disease, particularly characterized by polyarthritis. The inflammation is primarily located in the smaller joints of the hands and feet and is usually symmetrical. Although the onsef can be at any age, its peak onset is in the 4 th and 5th decade. RA is a disease that affects at least twice as many women as men. The detiology of RA appears to be multifactorial: genetic as well as environmental as immunalogic factors seem to play a role. RA is a common disease with a prevalence of approximately $1 \%$ in people of 40 years and older."

\section{Radiological joint damage and RA}

Persistent arthritis due to RA often leads to joint damage that can be visualized on radiographs. ${ }^{2}$ Plain radiography is currently still the standard method to assess radiologicall joint damage in patients with $R A$, in trials as well as in clinical practice. ${ }^{3,4}$ The abnormalities that can be seen on plain films due to RA are: soft tissue swelling, juxtaarticular and diffuse osteoporosis, subchondral cysts, joint space narrowing (caused by loss of cartilage), malalignment, subluxation, ankylosis, reactive sclerosis and osteophytes. ${ }^{5}$ Prevention of radiological damage is regarded as an important goal of RA therapy and recognized by the American Food and Drug Administration (FDA) as a separate claim. Radiological joint damage is consequently part of the core sets of outcome measures used to evaluate the disease-modifying effects of therapies for $R A$ in randomised clinical trials (RCTs) that have duration of 1 year or longer.

To be able to use plain radiographs as an outcome measure in trials, several scoring methods have been developed and evaluated. The methods developed by Sharp and Larsen and their modifications ${ }^{710}$ are most widely used at this moment. Despite the past validations, several methodological issues regarding the scoring of joint damage on plain films, however, still have to be solved."

\section{Thesis structure}

The facus of this thesis is the assessment of radiological joint damage due to RA on plain radiographs in RCTs by the Scott modified Larsen method and/or by the van der Heijde modified Sharp method. Several different methodological issues are discussed in this thesis.

In chapter 2, the reliability of the Sharp/van der Heijde and the Larsen/Scatt methad expressed as the discriminative capacity and responsiveness are 
compared by statistics based on the generalizability theory. This theory recognizes multiple sources of meosurement error within the same meosurement, so allowing different applications of the instrument to be evaluated simultaneously. ${ }^{\prime 2}$ Besides differences in reliability between the 2 scoring methods, also the issue of the optimal number of readers to be used when scoring films is addressed in this chapter. This chopler further discusses the pros and cons of the reliability statistics obtainable with generalizability analyses and the influence of the number of patients in the RCT on them.

In chapter 3, the use of different sub-scores of the Sharp/van der Heijde erosion score in early RA trials is discussed. Background of this chapter is that, in the context of a drug trial, prevention of erosions in undamaged joints is often considered more important than prevention of progression in already damaged joints, although liftle research on the consequences of excluding previously eraded joints has been done. Chapter 3 discusses this issue by assessing the relative weight of the different erosive sub-scores and their discriminative ability in a RCT.

In chapter 4, the assessment of the Minimal Clinically Important Difference (MCID) in joint damage for the Sharp/van der Heijde and the Larsen/Scot method by a clinical expert panel is discussed. Background of this chapter is that in order to give additional insight in the results of a trial, the number of patients actually responding to a particular drug is also often presented. To be able to do so, the continuous radiological damage scores have to be dichatomised and thus a valid and clinically relevant cutoff level should be chosen. This chapter discusses the results of a study in which the judgment of an international panel of rheumatologists is used to determine the MCID for the damage scores in four hypathetical clinical settings (short versus long disease duration and mild versus high disease activity). This study further discusses how the MCID relates to the so-called Smallest Detectable Difference (SDD). The SDD is presenting the $95 \%$ limits of agreement between two obsemotions leither made by the same observer iwice, or between two different observers). The SDD has been advocated to use as minimum cutoff level to determine whether the radiological

One part of the evaluation of the usefulness of expert panels like the one used in this chapter is to assess the consistency of the panel's judgments. Another aspect that should be investigated is the validity of the panel, which is ideally done by comparing the results with a sa-called gold standard. Without the availability of a gold standard, the scores can alternatively be compared with the judgments of a panel composed of panellists of another discipline. In chapter 5 the judgments of the panel of rheumatologists mentioned in chapter 4 are compared with the
judgments of a couple of experienced musculoskeletal radiologists.

Chapter 6 discusses the influence of knowing the sequence of films on the detection of clinically relevant changes in radiolagical jaint damage. 
Background to this chapter is that a former study showed that reading with known sequence of the films (chronological reading) enhanced the sensitivity to change of the scoring method compared to reading films grouped per patient withoul knowledge of the sequence (paired reading). 15 it could however not be excluded that this was primarily caused by expectation bias rather than the diminishing of measurement error. In order to assess if the extra signal picked up by reading in chronolagical order was principally bias or indeed a more precise signal, a study was performed to determine the influence of the reading order on the ability of the Sharp/van der Heijde and Larsen/Scott scoring method to detect clinically relevant progression of radiological damage in the individual patient defined by an expert panel as external criterion.

As mentioned above, the smallest defectable difference (SDD) has been advacated to use as minimum cutoff level for reporting patients with and without radiographic progression of joint damage. However, in scoring radiological joint damage due to RA, progression of damage is usually based on simultaneous assessment of series of films from an individual patient (paired or chronological reading) as described above. If films are read in such a setting, the Smallest Detectable Change (SDC) appears to be more appropriate to use as cutoff level. Chapter 7 presents how to assess the SDC and discusses why the SDC is under this condition more appropriate to use as cutoff level than the SDD.

In order to increase the contrast between an intervention and a control group, RCTs often include patients with a high prior risk on the outcome of interest by selecting on baseline predictors for that particular outcome. One of the strongest predictors for further progression of radiological joint damage is joint damage present at baseline. As a consequence one could expect that to reach the same power for a RCT, fewer patients would be needed in case of radiological joint damage as outcome if patients were selected for the presence of baseline damage. Chapter 8 discusses the observation that this is not necessarily true under all circumstances. Statistical power for dichotomous outcomes appears to be determined by a critical relationship between prior risk and on how a particular treatment exents its efficacy with respect to this prior risk.

Chapter 9 discusses a study performed to evaluate whether plain radiographs are able to reveal sufficient changes in joint damage due to RA within a 3 months interval to provide an adequate contrast between the treatment groups to detect clinically and statistically significant differences. Background of this chapter is that FDA guidelines request that phase 3 trials should be at least one year in duration in order to consider a claim of structural damage. Phase 2 (dose finding) trials are however much sharter of duration, often 3 months or less and therefore structural damage is never included as outcome in these trials. If it would be possible to detect progression of joint damage within the 3 months of a phase- 2 trial this would have clear advantages. The optimal doseand exposure range for slowing progression of radiologicall joint damage could for instance then be defined in a very early stage. 
Chopter 1

In chapter 10 the results and conclusions of the previous chapters are summarized and the implications and recommendations for future research discussed. 


\section{References}

1. Duncan AG. Clinical features of rheumatoid arthritis: early, progressive and late disease. In: Klippel $\mathrm{JH}_{*}$ Dieppe $\mathrm{PA}_{*}$ eds. Practical rheumatology. London: Fiona Foley, 1995:169-82.

2. van der Heijde $D$. De waarde van rontgenfoto's bij meumatoide arthritis. Ned Tijdschr Geneeskd 1997;141:1725-30.

3. Boers $M$. The validity of radiography as outcome measure in theumatoid arthritis. J Rheumatol 1995;22:1783-6.

4. Boers $M$. Value of magnetic resonance imaging in theumatoid arthritis? Lancet 2000;356: : 458 -9.

5. Sharp JT. Assessment of radiographic abnormallities in rheumatoid arthritis: what have we accomplished and where should we go from here? J Rheumatol 1995;22:1787-91.

6. Guidance for Industry. Clinical development for drugs, devices and biological products for the treatment of Rheumatoid Arthritis (RA). Rockville: Center for Drug Evaluation and Research, Food and Drug administration, 1997:1-56.

7. Sharp JT, Young DY, Bluhm GB, Brook A, Brower AC, Corbett $M$, Decker JL, Genant HK, Gofton JP, Goodman N, ef al. How many joints in the hands and wrists should be included in a scare of radiologic abnomalities used to assess rheumatoid arthritis? Arthritis Rheum 1985;28:1326-35.

8. Larsen A, Dale $K$, Eek M. Radiographic evaluation of rheumatoind arthritis and related conditions by standard reference films. Acta Radiol 1977;18:481-91.

9. Scott DL, Houssien DA, Laasonen L. Proposed modification to Larsen's scoring methods for hand and wrist radiographs. $\mathrm{Br} \|$ Rheumatol 1995;34:56.

10. van der Heijde D. How to read radiographs according to the Sharp/van der Heijde method. J Rheumatol 2000;27:261-3.

11. van der Heijde DM. Plain X-rays in theumaloid arthritis: overview of scoring methods, their reliability and applicability. Baillieres Clin Rheumatol 1996;10: 435.453 .

12. Shavelson RIJ, Wenn NM. Generalizability theory: a primer. Newbury Park London New Delh: SAGE publications, 1991.

13. Lassere $M$, Boers $M$, wan der Heijde D, Boonen A, Edmonds $\downarrow$, Saudan A, Verhoeven A. Smallest detectable difference in radiological progression. I Rheumatol $1999 ; 26: 731-9$.

14. van der Heijde D, Simon L, Smolen J, Strand V, Sharp J, Boers M, Breedveld F, Weisman $M$, Weinblatt $M$, Rau $\mathbb{R}$, Lipsky $P$. How to report radiographic data in randomized clinical trials in theumatoid arthritis; guidelines from a roundiable discussion. Arthritis Rheum 2002;47:215-8.

15. van der Heijde D, Boonen A, Boers $M$, Kostense P, wan Der Linden S. Reading radiographs in chronological order, in pairs or as single films has important implications for the discriminative power of rheumataid arthritis clinical frials. Rheumatology (Oxford) 1999,38: 1213-20. 


\section{Chapter 2}

The Sharp/van der Heijde method out-performed the Larsen/Scott method on the individual patient level in assessing radiographs in early rheumatoid arthritis

Bruynesteyn $K$, van der Heijde D, Boers $M$, van der Linden Si. Lassere $M$, wan der Vleuten C

J Clin Epidemiol, accepted for publication 


\section{Abstract}

\section{Aim}

To test the reliability of 2 radiological scoring methods in theumatoid arthritis (RA) - the Sharp/van der Heilde (SWH) and the Larsen/Scott (LS) - with generalizability analyses.

\section{Methods}

Films of 51 patients representing the spectrum of early RA were read by 2 raters for each method. The discriminative ability and responsiveness were expressed as: intraclass correlation coefficients (ICCs), 2 types of smallest detectable difference (SDD) and 2 types of smallest detectable change (SDC); reflecting measurement error when discriminating between or defecting changes within 1) individuals or 2) groups They were calculated for (average) scores of $1-3$ raters.

\section{Results}

The discriminative capacity $(0.85 .0 .97)$ and responsiwervess $(0.91-0.97)$ were good when expressed by ICC. On group level the SDDs and SDCs ranged between $0.6 \% .3 .3 \%$ of the max. obtainable score. On individual level, the scores showed better reliability measured with the SWH (SDDs 2.0\%-3.4\%) than with the LS (SDDs 5.3\%-9.2\%). The SVH also assessed changes in scores in individuals with less measurement error (SDCs $1.3 \%-2.2 \%$ ) than the LS (SDCs $2.3 \%-3.9 \%)$.

\section{Conclusion}

For early RA patients, the SwH seems preferable if analyses on individual level are included. 


\section{Introduction}

Persistent arthritis caused by the chronic inflammatory disease rheumatoid arthritis (RA) often leads to joint damage. This can be visualized by $X$-rays as erosions and joint space narrowing. Prevention of radiological damage is regarded as an important goal of RA therapy and recognized by the American Food and Drug Administration (FDA) as a separate claim. The most widely used radiological scoring methods to quantify the joint damage in clinical trials or cohort studies are the Sharp and the Larsen methods and their modifications. ${ }^{1-7}$ They are used to detect changes over time within patients or groups of patients (as an evaluative instrument) as well as to detect differences between individual patients or groups of patients (as a discriminative instrument). ${ }^{8}$ The sensitivity to detect changes over time (= responsiveness) and the ability to discriminate between patients are both part of the reliability of these scoring methods and have been evaluated several times within the last decades by various statistics. ${ }^{4,9-21}$ This variability in methods does not allow an unequivocal comparative evaluation of radiological scoring methods.

Reliability of a method is mostly expressed by intra class correlation coefficients (ICCs). Besides the ICC, reliability analyses increasingly include statistics such as the standard error of measurement $(\text { SEM) })^{22-25}$ or the smallest detectable difference $(\mathrm{SDD})^{26 \cdot 28}$, which have the advantage that they express measurement error in the metric unit of the measurement. There is fewer consensus on which statistic to use to evaluate a measure or method's responsiveness. Standard effect sizes (SES), standard response means (SRM) or Guyatt responsiveness ratios $^{29}$ are often calculated ${ }^{30-32}$, but also ICCs can be used to express the sensitivity to detect changes. ${ }^{33}$ Advantages of also using an ICC or related statistics as responsiveness measurement are that both aspects of reliability of the measurement - the ability to discriminate between patients and the sensitivity to detect changes over time - are expressed in a similar manner and can be compared directly, especially when they are calculated on the same data.

ICCs and related statistics are originally based on the classical test theory, a measurement theory originating from the social sciences and developed to estimate the reliability of scores derived from measures. ${ }^{34}$ Later on, this theory was extended by generalizability theory. ${ }^{35-38}$ This theory recognizes multiple sources of measurement error (i.e., noise) within the same measurement. In accordance with this, multiple sources of measurement error can be estimated within the same ANOVA design, thereby allowing different applications of the instrument to be evaluated simultaneously. Reliability statistics based on the classical test theory have been assessed betore for several radiological scoring methods for RA. ${ }^{39}$ However, generalizability analyses have rarely been used. 4,20 This is unfortunate because generalizability theory offers several advantages over standard measurement approaches, as we shall demonstrate. 
minimal jaint damage at baseline. Radiographs for this study were selected by an independent rheumatologist (MB), not involved in the rating of the films, to represent the spectrum of early RA. The results are summarised in Table 2.1. The films showed a wide range of (relatively low) baseline scores for both the Sharp/van der Heijde (Inter Quartile Range 1.0-24.0) and the Larsen/Scott (IQR 2.5-24.0). The IQR in progression in scores were 3.0-17.0 and 1.5-17.5, respectively. The mean \pm SD and median baseline scores were $14.2 \pm 16.0$ and

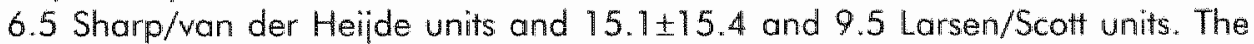
mean \pm SD and median progression in scores were $13.0 \pm 13.0$ and 7.5 Sharp/ van der Heijde units and $9.2 \pm 9.6$ and 5.0 Larsen/Scott units, a distribution which is clearly not rectangular, but which reflects the skewed distribution of radiological joint damage as seen in the field.

TABLE 2.1

Radiographic distribution of characteristics of the study population according to the Sharp/wan der Heijde and the Larsen/Scott scoring method $(n=51)$

\begin{tabular}{|c|c|c|}
\hline & $\begin{array}{l}\text { Sharp/wan der Helide } \\
\text { (maximal score 448) }\end{array}$ & $\begin{array}{l}\text { Larsen/Scolt } \\
\text { Inaminal score } 200\end{array}$ \\
\hline \multicolumn{3}{|l|}{ Baseline scores } \\
\hline Mean $\pm S D$ & $14.2 \pm 16.0$ & $15.1 \pm 9.5$ \\
\hline Median & 6.5 & 9.5 \\
\hline Interquartile range & $1.0 \cdot 24.0$ & $2.5-24.0$ \\
\hline Full range & $0.0-58.5$ & $0.0-56.5$ \\
\hline \multicolumn{3}{|c|}{ Progression of the scores in $1 \mathrm{yr}$} \\
\hline Mean $\pm S D$ & $13.0 \pm 13.0$ & $9.2 \pm 9.6$ \\
\hline Median & 7.5 & 5.0 \\
\hline Interquartile range & $3.0-17.0$ & $1.5-17.5$ \\
\hline Full range & $0.0-50.5$ & $0.0-39.5$ \\
\hline
\end{tabular}

\section{Statistical analysis}

1) Generalizability theory

\section{G-study}

Generalizability $(G)$ theory uses a factorial ANOVA model to evaluate multiple potential sources of measurement error by partitioning the total variance of the data into components originating from different independent sources and their interactions. In generalizability theory, sources of variation are called "facets" (analogous to the term "factors" in regular ANOVA analyses) and the levels of these facets are called "conditions". In this study there are 3 main facets for each method: patient (5) conditions), rater (2 conditions) and time (2 
Object of this study was to compare the ability (1) to discriminate between patients or groups of patients and (2) to defect change in 1 year $11 \mathrm{yr}$ responsiveness) of 2 radiological scoring methods, namely the van der Heijde modified Sharp method and the Scott modified Larsen method, by applying generalizability theory.

\section{Patients and Methods}

\section{Radiological scoring methods}

The Sharp/van der Heijde methad ${ }^{3}$ assesses erosions and joint space narrowing separately in the hands and feet and has a range from 0 to 448 . Thirty-two joints in the hands and 12 in the feet are scored for erosions, with a maximum score of 5 per jaint in the hands and 10 per joint in the feet. Joint space narrowing is graded from 0 to 4 in 30 joints in the hands and in 12 joints in the feet. The principal score used in the analyses is the fotal score, which is the sum of the erosion score and the joint space narrowing score. ${ }^{40}$

The Larsen/Scott method ${ }^{7.41}$ has a range from 0 to 200 if joint damage in the hands and feet is evaluated. The Larsen/Scott method assesses global damage per joint and scores are assigned by comparison with series of standard radiographs. These scores are mainly influenced by the erosive joint damage. Twenty joints in the hands and 10 joints in the feet are graded. The wrists are evaluated as single joints. Scores range from 0 to 5 and the score of the wrist is weighted by a factor 5 .

\section{Raters}

Radiographs were scored ance according to both the Sharp/van der Heijde and the Larsen/Scatt methods by 2 experienced raters for each method. In practice it is not possible to be an expert in both methods in the same study period. For each scoring method 2 different raters well trained in that particular method were therefore used to provide for each method optimum circumstances. The raters were blinded for patient's identity and treatment. The chronological sequence of the films was known by the 4 raters, because reading films in chronological order is most sensitive to change. ${ }^{20}$ Furthermore, knowledge of the chronological order led to an increase in defecting clinically relevant changes without serious overestimation of non-relevant differences in a recent
study. So, it was recommended that in trials, reading films should preferably be
done with knowledge of the chronological order ${ }^{2}$ ? done with knowledge of the chronological order. ${ }^{42}$

\section{Patients}

Baseline and 12 month radiographs of 51 early RA patients were selected from a randomized controlled trial with 2 active treatment arms (COBRA trial). ${ }^{43} A^{*}$ entry these patients fulfilled the 1987 ACR classification criteria for RA $A^{44}$ and had a disease duration of $<2$ years. The trial had a high number of patients with 
conditions). Following the ANOVA, variance components are estimated in which the sources of wariance (the main facets and their interactions) are expressed in their relative size to each other. Variance components are calculated out of the mean squares (MS) from the ANOVA tables. For the equations we refer to the liferature. $3 x, 45$ The variance component associated with a facet reflects how much that particular facet contributes systematically to the total wariance of the measurement results. Comparison of the sizes of each variance component thus reflects the relative ranking of the sources of variance. The variance component of an interaction reflects how much the contribution of a particular facet depends on the different levels of the other facet(s) (and vice versa). Which (combination of) variance components constitute measurement error (noise) depends on the study questions. Table 2.2 lists the variance componenis estimated from the two 3-way AVOVA used in this study and their interpretations. The computer program GENOVA for PCs by Crick and Brennan ${ }^{46}$ ran the 3-way ANOVA for each methad and estimated the variance components.

TABLE 2.2

Interpretation of components of variance in the ANOVA model patient $x$ rater $x$ time

\begin{tabular}{|c|c|c|}
\hline Source of vartance. & $\begin{array}{l}\text { Symbol in } \\
\text { formula }\end{array}$ & Description \\
\hline Potient & $\hat{\sigma}^{2}(p)$ & $\begin{array}{l}\text { Variance caused by variation in status scores } \\
\text { between patients }\end{array}$ \\
\hline Rater & $\hat{\sigma}^{2}(r)$ & $\begin{array}{l}\text { Variance caused by variation in status scores } \\
\text { betweem raters }\end{array}$ \\
\hline Time & $\hat{\sigma}^{2}(t)$ & $\begin{array}{l}\text { Variance caused by wariation in status scores within } \\
\text { patients due to the elapse of fime (1 year) }\end{array}$ \\
\hline Patient * Rater & $\hat{\sigma}^{2}(p r)$ & $\begin{array}{l}\text { Variance caused by the fact that the wariance due } \\
\text { to the variation between raters depends on the } \\
\text { patient in question (and vice versa) }\end{array}$ \\
\hline Patient "Time & $\hat{\sigma}^{2}(p l)$ & $\begin{array}{l}\text { Variance coused by the fact that the variance due } \\
\text { to the elapse of time depends on the patient in } \\
\text { question (and vice versa) } \\
\text { Variance consed }\end{array}$ \\
\hline Rater * Time & $\hat{\sigma}^{2}(n)$ & $\begin{array}{l}\text { Variance caused by the fact that the variance due } \\
\text { to the variation between raters depends on the } \\
\text { fime point of the } X \text {-ray (and vice versal }\end{array}$ \\
\hline Residual error & $\hat{\sigma}^{2}(p r t, e)$ & $\begin{array}{l}\text { That residual part of the wariance not explained by } \\
\text { the investigated sources of variance. }\end{array}$ \\
\hline
\end{tabular}

D-study

After the variance components of potential sources of error have been estimated in a Generalizability study, a so-called Decision (D) study follows. In D studies 
different applications of the measurement instrument can be evaluated, e.g., (expressed in G-theory terms) making absolute or relative decisions and/or generalizing over the whole domain of conditions of a facet or not (i.e., treating facets as fixed or random). For a more detailed explanation of relative vs. absolute decisions and fixed vs. random facets and, moreover, its general implications for the calculation of the measurement error we refer to the literature. ${ }^{37,38,45}$ In this study, all variance components and their interactions were in principle included in the measurement error because we wished to generalize over the absolute scores ("making absolute decisions) and we wanted to account for differences between raters beyond those included in the study (treating the facets as random). We evaluated the following applications of the scoring methods: 1) the ability to discriminate between the status score of individual patients and between the mean status scores of groups of patients, 2) the ability to defect changes within patients or groups of patients and 3) the ability to discriminate between detected changes over time.

\section{2) Statistics used (see formulae in appendix)}

The following statistics were calculated:

- Reliability ICC of the status scores that express the ability of the methods to discriminate between status scores of 2 different patients as ratio of the true signal variance (status score) to the total variance.

- Responsiveness reliability ICC that express the ability to detect changes over 1 year as a ratio of the true signal variance (change in status score) to the "total" variance.

- SDDs that express the measurement error when discriminating between status scores of 2 different patients or 2 groups of patients. If a difference in scares between 2 patients or 2 groups of patients is larger than the accompanying SDD, it exceeds the 95\% confidence interval of the measurement error. Such a difference is then regarded as "real".

- SDCs (smallest detectable changes) that express the measurement error when measuring change over 1 year. If a change is larger than the accompanying SDC, it exceeds the $95 \%$ confidence interval of the measurement error. Such a change in then regarded as "real".

- Reliability ICC based on change-scores that express the ability to discriminate between changes over time.

- SDD which express the measurement error when discriminating between 2 mean group changes.

\section{3) Influence of averaging the scores in a study}

In trials, mean scores of 2 or more raters are often used in the analyses. To evaluate the influence of averaging the scores of 2 or 3 raters on the reliability stalistics, the variance components related to the facet "rater" where divided respectively by the number 2 or 3 in the formulae for the ICCs, SDDs and SDCs. To estimate the reliability and sensitivity to change of the methods if using the mean scores of 3 raters, "mathematical simulations" were thus used in this 
study. This is valid under the assumption that a third reader would have the same experience and training.

4) Influence of the number of patients in the groups of a study

The influence of the number of patients included in a study on the SDD and the SDC within groups was also evaluated. This was done by dividing the variance components related to patients by fictitious simulated numbers of patients. The influence of the group size on the SDD and on the SDC was evaluated with 3 simulations: $n=50, n=100, n=150$.

\section{Results}

Table 2.3 presents the variance components, their standard errors and each facet's contribution to the total variance for the scores of the Sharp/van der Heijde and Larsen/Scatt method. The major proportion of variability of the status scores was accounted for by the between-patient variation; $61 \%$ for both the Sharp/van der Heijde and the Larsen/Scott scores. Other major contributors to the total variance were the facet time $117 \%$ and $13 \%$, respectively) and its interaction with the facet patient $(16 \%$ and $13 \%$, respectively). This means that patients' scores changed considerably over time, and these changes over time were very different for different patients. Furthermore, the variance between patients exceeded the variance within. The systematic variation in scores due to the raters was negligible for both scoring methods $(<1 \%)$. Nevertheless, there were differences between the raters. This variation due to the raters depended on the patient, especially when scoring according to the Larsen/Scott method ("patient * rater"; $4 \%$ for Sharp/van der Heijde versus $11 \%$ for Larsen/Scott). On the other hand, the variance due to the Sharp/van der Heijde or the Larsen/Scott raters was barely influenced by the facet time ("rater * fime"; both $<7 \%$ ), so the difference between the raters did not change between baseline and 12 months. The residual (unexplained) error was relatively small: $2 \%$ of both the Sharp/van der Heijde and the Larsen/Scott scores. The standard errors (SE) of the facets reflect the precision of the variance component estimates. They were relatively large due to the small number of conditions of the facets. Finally, to check the robustness of the ANOVA, the model was repeated with logarithmic transformed data. The results showed that the relative sizes of the variance components expressed as \% of the total variance were almost identical to those 
TABLE 2.3

Variance components, their standard errors and percentage of total woriance for each scoring method

\begin{tabular}{|c|c|c|c|c|c|c|}
\hline \multirow[b]{2}{*}{$\begin{array}{l}\text { Vananice } \\
\text { components }\end{array}$} & \multicolumn{3}{|c|}{ Sharp / van der Heilde method } & \multicolumn{3}{|c|}{ Larsen / Scotl method } \\
\hline & $\begin{array}{l}\text { Variance } \\
\text { estimate } \\
\left(\hat{\sigma}^{2}\right)\end{array}$ & $\begin{array}{c}\text { Standard } \\
\text { Error }\end{array}$ & $\begin{array}{c}\text { Percentage } \\
\text { of total } \\
\text { vanamce }\end{array}$ & $\begin{array}{c}\text { Vaniance } \\
\text { estimate } \\
\left(\sigma^{2}\right)\end{array}$ & $\begin{array}{l}\text { Standard } \\
\text { Enror }\end{array}$ & $\begin{array}{l}\text { Percentiage } \\
\text { of total } \\
\text { vaniance }\end{array}$ \\
\hline Potient & 297.92 & 69.03 & 61 & 196.71 & 46.92 & 61 \\
\hline Rater & 0.00 & 0.62 & $<1$ & 0.00 & 0.25 & $<1$ \\
\hline Time & 82.29 & 68.90 & 17 & 41.21 & 34.38 & 13 \\
\hline Patient* Rater & 17.38 & 4.74 & 4 & 35.48 & 7.77 & 11 \\
\hline Patient Time & 79.11 & 16.74 & 16 & 41.75 & 9.00 & 13 \\
\hline Rater * Time & 0.82 & 0.87 & $<1$ & 0.00 & 0.13 & $<1$ \\
\hline Residual error & 12.05 & 2.36 & 2 & 7.92 & 1.55 & 2 \\
\hline
\end{tabular}

The reliability coefficients of the status scores for both methods are given in Table 2.4, assessed for 3 different study applications: studies with only 1 rater, and studies applying the mean scores of 2 respectively 3 raters. The reliability coefficients were all highly acceptable, the Sharp/van der Heijde coefficients being somewhat higher than the Larsen/Scott coefficients. The reliability of the status scores of the Sharp/van der Heijde and the Larsen/Scott method expressed as SDDs are presented in Figure 2.1a and 2.1b. The measurement error of the Sharp/van der Heijde if discriminating between individual status scores, expressed as absolute value, was smaller than the estimated error of the Larsen/Scott method. Expressed as ratio of the SDD to the maximal obtainable score the difference between the methods was even greater. On group level, the measurement error was smaller for the Larsen/Scott method; however, the ratio of the group-SDDs to the maximal obtainable score was still smaller for the Sharp/van der Heijde than for the Larsen/Scott.

TABLE 2.4

Reliability coefficients of the status scores of 2 scoring methods; Estimated for studies in which either the scores of 1 rater or the mean scores of 2 or $3^{*}$ raters would be used

\begin{tabular}{lcc}
\hline Reliability coefficient & Sharp/ van der Herlde & Larsen/Scot \\
1 rater & 0.93 & 0.85 \\
2 raters & 0.96 & 0.92 \\
3 raters & 0.97 & 0.94 \\
\hline
\end{tabular}

simulated data, under the assumption hat a 3 r rater would be equally trained and experienced 

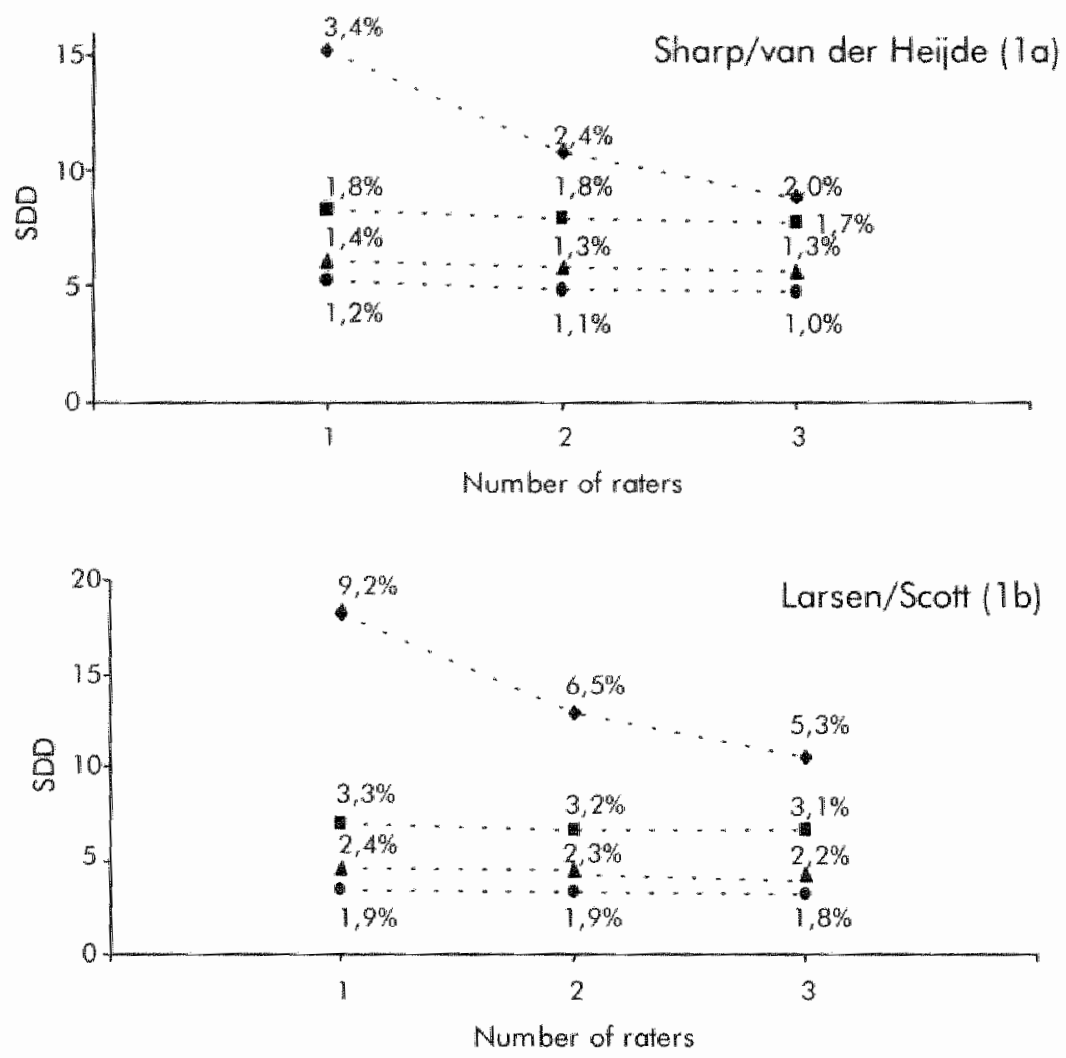

FIGURE $2 a$ and $2 b$.

Reliability of the Sharp/van der Heijde (1 a) and Larsen/Scott status (1 b) scores expressed by 2 types of Smallest Detectable Differences (SDDs between individuals and between groupl, estimated for studies in which either the scores of 1 rater or the mean scores of 2 or 3 raters would be used.

-.. SDD when discriminaling between status scores of individuals fformula see method section);

-. $=$ SDD between mean status scores of groups with per group 50 patients (simulated data; formula see method section)

$\ldots . . .=$ SDO between mean status scores of groups with per graup 100 patients

-... $=$ SDD between mean status scores of groups with per graup 150 patients

The percentage presented is the ratio of the SDD to the maximal obtainable score ( 448 and 200 , respectively!.

The responsiveness statistics are given in Table 2.5 and the Figures 2.20 and $2.2 b$. The responsiveness reliability coefficients were good for both the Larsen/Scott and the Sharp/van der Heijde method. On the other hand, the 
measurement error when detecting individual changes over time was actually relatively larger if the data was scored with the Larsen/Scott $(2.3 \%-3.9 \%)$ than with the Sharp/van der Heijde (1.3\%-2.2\%). The SDCs of the Larsen/Scott, diminished, however, substantially when changes on group level were analysed $(0.8 \%-1.4 \%)$, becoming comparable with the group-SDCs of the Sharp/van der Heijde method $(0.6 \%-1.0 \%)$.

\section{TABLE 2.5}

Responsiveness reliability coefficients of 2 scoring methods; Estimated for studies in which either the scores of 1 rater or the mean scores of 2 or $3^{\prime \prime}$ raters would be used

\begin{tabular}{lcc}
\hline & Sharprwan der Heilde & \\
\hline Responsiveness coefficient & & Larsen/Scott \\
1 rater & 0.93 & 0.91 \\
2 raters & 0.96 & 0.95 \\
3 raters & 0.97 & 0.97 \\
\hline
\end{tabular}

* simulated data, unider the assumption that a $3^{\text {rd }}$ rater would be equally trained and experienced

From Figure $2.2 a$ the "SDD of mean changes over time between 2 groups" can be calculated as 6.4 Sharp/van der Heilde units $\left(4.5^{*} \sqrt{ } 2,1.4 \%\right.$ of the maximal obtainable score) if analysing groups with $n=50$ with the scores of 1 rater. If the mean scores of 3 raters would be used and the groups would consist of 150 patients instead of 50 this becomes 3.6 Sharp/van der Heijde units $(2.5 * \sqrt{2}$, $0.8 \%)$. The corresponding SDDs of the Larsen/Scott were $3.9(2.0 \%)$ and 2.1 $(1.1 \%)$, respectively (calculated from Figure 2.4). The reliability coefficients of the change-scores of the Sharp/van der Heijde and the Larsen/Scott were comparable. The reliability coefficients of change-scores of the Sharp/van der Heijde method were $0.86,0.92$ and 0.95 if using the scores of 1,2 , or 3 raters respectively. Those of the Larsen/Scott method were $0.84,0.91$ and 0.94 . Thus, when comparing the reliability coefficients of the Sharp van der Heijde changescores with those of the status scores, the first was slightly lower when using the scores of 1 rater $(0.86$ versus 0.93$)$. However this difference was practically nullified when using mean scores of $2(0.92$ versus 0.96$)$ or 3 raters $(0.95$ versus. 0.97 ). The reliability coefficients of the Larsen/Scott change-scores and the coefficients of the status scores were all comparable

Using mean scores of 2 ar more raters enhances the reliability of a method; using the mean scores of 2 or 3 raters enhanced both ICCs (Table 2.4 and 2.5). The SDDs and SDCs also diminished remarkably when using mean sores. The effect of adding more raters on the group-SDCs was however much smaller and even almost negligible when looking at the measurement error when discriminating between mean status scores on group level, the group-SDDs (Figures 2.1 and 2.2). 

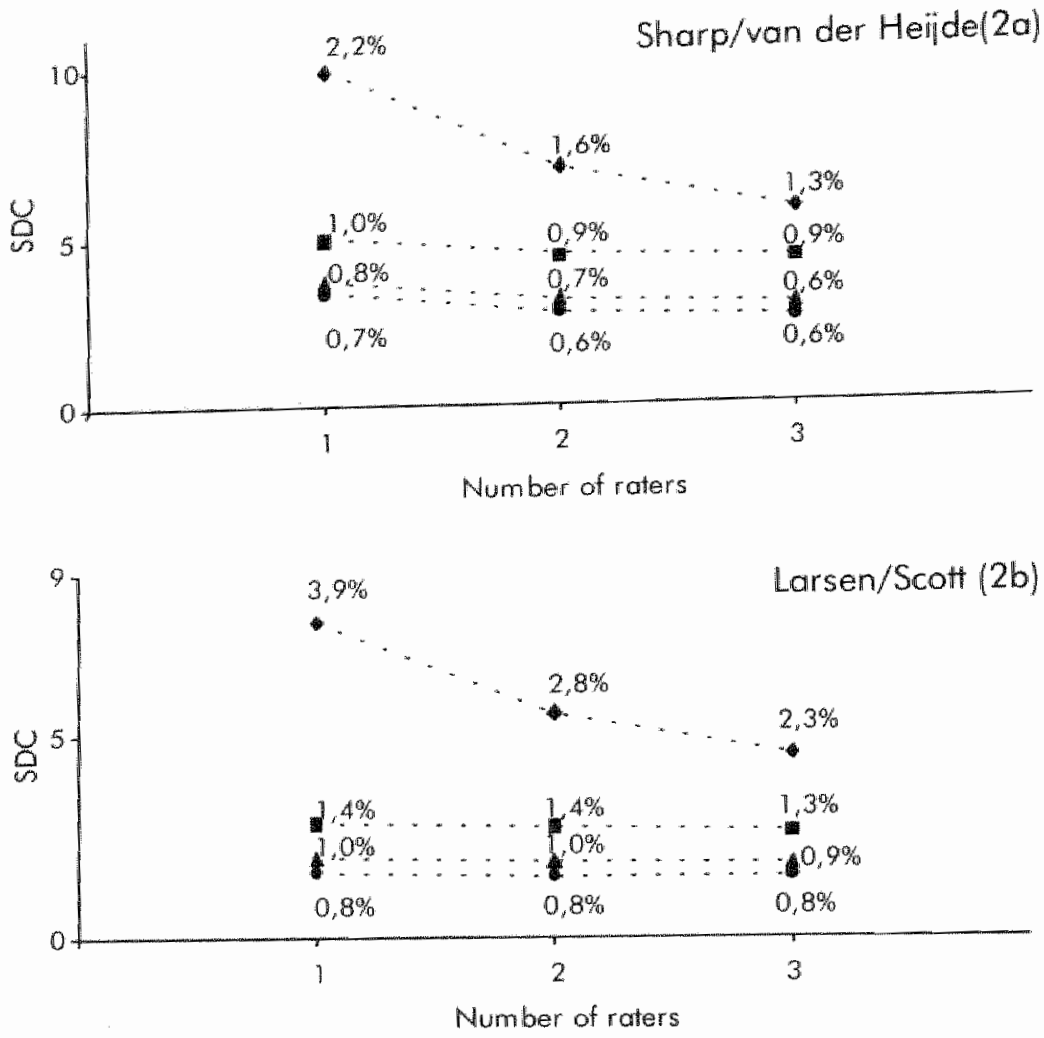

FIGURE $2 a$ and $2 b$.

Responsiveness of the Sharp/van der Heijde (2a) and the Larsen/Scatt (2b) method expressed by 2 types of Smallest Detectable Changes (SDCs of individuals and groups), estimated for studies in which either the scores of 1 rater or the mean scores of 2 or 3 raters would be used

... = SDC of indiwiduals (formula see method section):

-. = SDC of mean status scores of groups with per group 50 palients (simulated data; formula see method section)

.... = SDC of mean status scores of groups with per group 100 patients

.... $=$ SDD of mean stotus scores of groups with per group 150 patients

The percentage presented is the ratio of the SDC to the maximal abtainable score 1448 and 200, respectively).

If a trial would include 100 or 150 patients instead of 50 per (treatment) group this would also result in a distinct decrease of measurement error. The effect of adding 50 patients per group on the SDD between mean status scores was clearly greater than the effect of adding a second or third rater (Figure 2.1 a and $2.1 b)$. The effect of using 2 or 3 raters instead of 1 on the SDCs on group-level 
was however almost comparable with the effect of adding 50 patients per group (Figure 2.2a and 2.2b).

\section{Discussion}

The generalizability analyses in this study confirm the discriminative capacity and responsiveness of the 2 methods commonly used to score radiological progression in RA, the Sharp/van der Helide and Larsen/Scolt methods. Furthermore, the study is a demonstration of the value of generalizability theory in the assessment of discriminatory capacity and responsiveness of measures: it provides ICCs, SDDs and SDCs for a variety of cross-sectional and longitudinal contexts. This is important because the sole use of ICCs as method of evaluating reliability can give incomplete and potentially misleading information.

The measurement error of the Sharp/van der Heijde method when discriminating between individual status scores (SDD between individuals) was considerably smaller than the measurement error of the Larsen/Scott method. The measurement error when measuring individual changes in scores over time (SDC within an individual) was relatively speaking also smaller for the Sharp/van der Heijde when comparing with the Larsen/Scott. However, when changes over time were analysed on group level they were practically equal. Nevertheless, results on individual patient level are included increasingly in analyses of clinical trials ${ }^{3,49.51}$, because they can add valuable information. For analysis on the individual level le.g., number of patients with an increase or decrease in joint damage) a cutoff level should be determined. This cutoff level should be equal to or larger than the SDC, since changes smaller than the SDC cannot be distinguished reliably form zero. In such situations the Sharp/van der Heijde method seems preferable. A possible explanation for the relatively larger measurement error of the Larsen/Scott method can be the fact that the Larsen/Scott method applies global scores instead of separate scores for erosions and joint space narrowing with less detailed descriptions on which grade to apply.

On first sight, the differences in measurement error found between the 2 scoring methods seems to be trivial at a couple few percent of the maximal oblainable scores. Other studies however have demonstrated a constant average annual increase of joint damage between the $1.6 \%$ and $1.9 \%$ of the maximal damage in the first 20 years, irrespective of which scoring system was used. ${ }^{52}$ So, even 20 years after diagnosis still only $40 \%$ of the maximum possible scores are used. Furthermore, a change of $1 \%$ in individual damage score, irrespective of which scoring system used, was regarded as clinically important by an expert panel of rheumatologists. ${ }^{53}$ Consequently, the small differences in percentages of the maximum obtainable scores found in this study, in favour of the Sharp/van der Heijde method, seem significant.

Comparison of the SDCs over time with the SDDs between status scores of patients showed that the actual measurement error was smaller when assessing 
changes in time than when measuring differences bewween patients. Furthermare, by multiplying the SDDs of change over time from Table 2.5 by $\sqrt{ } 2$, the measurement error when discriminating between change-scores of 2 (groups of) patients was assessed. These SDDs of changes over time between 2 (groups of potients were still smaller than the SDD between the status scores of 2 (groups of) patients. This shows that the raters agreed more on the progression of scores than on the status scores selves. The fact that successive radiographs of one patient were read simultaneously and with known sequence, i.e., not judged independently, will probably have played a considerable role. In fact the procedure of reading successive films of one patient simultaneously was introduced specifically with the measurement error in mind. Because in general SDDs of changes over time between 2 groups provide information for power calculations based on pragressors / nom-progressors, these "SDDs of changes over time between 2 groups" are useful parameters to present in reports.

The sizes of the SEs of the variance components (Table 2.3) correlated with the sizes of their accompanying variance components, except for the SE of the variance component of the focet time. In a design like ours, the SE of the facet "time" will awways be disproportional large, irrespective of the sample size. This is because it has only 1 degree of freedom, since there are only 2 conditions that determine the facet. The other SEs were considerable due to the rather small samples used in this study. Likewise, the assessment of the variance components of the facet "rater" is limited by studying only 2 raters in this study. In the classical test theory it is very common to calculate an interobserver reliability coefficient from the observation of only 2 raters, however in generalizability theory it is not common to observe only 2 conditions of a facet's universe. The high concordance between the relative sizes of the variance components of the 2 different rater couples (expressed as \% of the total variance in Table 2.3 ) is however a support for the reliability of the results of this study. Brennan, as "distributional-form assumptions are not made in defining or considered to be robust for in the model".5.4 Furthermore, ANOVAs are radiological jaint damage due to deviations from normality. However, since relative sizes of the variance comp $\mathrm{RA}$ is rather skewed, we decided to verify if the model with log-transformed data. The would not alter by ruming an ANOVA belief of the applicability of the $G$ theory for skewed datmost results strengthened our Fries et al. ${ }^{55}$ investigated the $G$ theory for skewed data.

1986 on alternative methods, which can be considered of experience already in now used scoring methods. This study showed that the level forerunners of the training is critical for the level of reliability. using statistics based on the classical
test theory. This was true for strategies that used separate scores for the and joint space narrowing (like our Shar used separate scores for the erosions strategies that used global scores (like Shar / used well-trained and experienced our Larsen/Scott method). We therefore Larsen/Scoth naters were well trained and for both methods. Although our excluded that the large variance 
found for this method was caused by the choice of the raters and is not inherent to the scoring method itself. Previously reported ANOVA of Sharp/van der Heijde data assessed by a different rater couple were consisted with our data and also showed no substantial "patient " rater" interaction. ${ }^{20}$ Information on the "pafient* rater interaction" of other couples of Larsen/Scott raters has, however, to our knowledge, not been published. But the reliability of the Larsen/Scott method has been analysed in several studies by other statistics. ${ }^{2} 12,41$ The Sharp/van der Heijde and the Larsen/Scoll scoring method are both designed to assess joint damage in clinical trials or cohort studies. They are both time-consuming methods, the Larsen/Scott being less time consuming than the Sharp/van der Heijde method. For clinical trialls, this should in general not be a problem. However, in daily practice, they are not feasible, because they are time-consuming and require trained raters. For clinical practice a simplified scoring method, the SENS, has been developed. ${ }^{4}$

The choice of patients influences reliability statistics. A wide range of patient scores influences ICCs: it enlarges the variance caused by variation between the patients i.e., the signal. So, when comparing the (very good) ICC from this study with ICCs of other studies, special attention must be paid to the range of scores in these studies. The actual measurement error, expressed by the SDD, does not tend to be smaller when measuring a broad spectrum of patients. Moreover, in studies with more baseline damage and more radiological progression per year the measurement error tends to be larger. ${ }^{12,39,56}$ The results found in this study are based on early RA patients and we don't know if the results can be generalized to trials that include patients with longer disease duration and thus more baseline damage. Note that when measurement error within a study is related to the amount of progression of joint damage, the assumption of homoscedasticity is violated. In such cases it is inappropriate to simply express measurement error in the actual units of measurement. Rather, one should use expressions that account for this relationship, e.g., the coefficient of variation. ${ }^{57}$ The fact that measurement error tends to be larger in studies with more baseline damage and radiological progression can explain why the SDDs found in our study are relatively large compared to some found in other studies with the same rater(s) . $^{39,40,42}$ Furthermore it should be noted that many of the SDDs reported are assessed by the so-called Bland and Altman repeatability coefficient ${ }^{\text {sB }}$ and not by generalizability analyses. This method is indented for test-retest analyses and does not take systematic bias into account. Moreover, studies employing this method usually only assess the measurement error when detecting differences in status or change scores between 2 patients (SDDs of status or change over time between 2 patients) ${ }^{12}$ rather than the measurement error when detecting change over time within a patient (SDC over time).

As Streiner and Norman ${ }^{33}$ already put it: "reliability of a measurement instrument is intimately linked to the population to which one wants to apply the measure. There is literally no such thing as the reliability of a test". The fact that the reliability is not context free is thus not limited to radiological scoring methods. ${ }^{12,39,56}$ Because of this, trial reports should ideally also give the reliability statistics in their particular contex". Moreover, reporting the actual variance 
components would be useful. This would increase insight into the properties of the scoring methods and would potentially enable pooling of variance components across different studies where appropriate.

This study also analysed the benefit of using mean scores of 2 or more raters. The discriminatory and evaluative capacity of the scoring methods did not change substantially on group level by adding 1 or 2 raters, given that these readers had the same training and experience. The reliability and responsiveness of the individual scores, on the other hand, did increase by adding raters. Further, the analyses revealed that the measurement error when using the means of 3 Larsen/Scatt raters still exceeded that of 2 Sharp/van der Heijde raters in percentages of the maximum score when assessing changes over fime. Even more, the SDD between Larsen/Scott status scores if using the means of 3 Larsen/Scott raters remained in percentage lower than the SDD of the Sharp/van der Heijde using 1 rater. The influence of averaging the scores of one or more raters on the reliability of progression scores has been investigated " to our knowledge, once before by Fries et al. ${ }^{55}$ They found that the greatest gains in reliability parameters were made by increasing the number of raters from 1 to 2, similar to our results on the reliability and responsiveness parameters of the individual scores. Furthermore, they concluded that adding more raters introduced extra costs with (relatively) little gain.

The group-SDDs estimated for the groups with $n=50, n=100$ and $n=150$ demonstrated the ability of generalizability theory to evaluate the hypothetical influence of the group sizes on the measurement error of a measurement. Moreover it makes it possible to compare the influence of enhancing the group size against the benefit of using the mean scores of more than one condition of a facet, here demonstrated by using the mean scores of 2 or 3 raters. In this study, it seemed that the benefit of including more patients was higher than the benefit of using more raters regarding the measurement error when discriminating between mean group status scores. However, the effect of adding more patients will probably not be unlimited. At some point the effect is expected to reach a ceiling at which an extra rater will result in a larger decrease of measurement error then when adding more patients. Furthermore, concerning the changes of scores over time the benefit of adding 50 patients was already
almost equal to the benefit of using 2 instead of 1 rater. In summary, this article provided more insight in the properties of the Sharp/van der Heijde and Larsen/Scott scoring methods and demonstrated the surplus reliability of a measurement especially the ability to assess simultaneously the Generalizability theory gives insight in the for several different applications. more thorough decisions can be made about thources of variance and so design a study. Briefly, the discriminative capacity and responsiveness were highly acceptable
for both scoring methods when comparing mean group scores. However, when
comparing individual patients, the Sharp/ measured with greater precision and the method was also more sensitive in
detecting change over 1 year. 


\section{Appendix}

For abbreviations see Table 2.2; SEM stands for the Standard Error of Measurement ${ }^{26}$ and the RMSE stands for the Root Mean Square Error ${ }^{47.48 ;} n_{\text {i }}$ represents the number of raters, $n_{p}$ the group size of the groups one wishes to compare and $n_{t}$ the number of time periods.

Ability to discrimination between patients

Formulae:

Reliability coefficient of status scores:

$$
\frac{\hat{\sigma}^{2}(p)+\hat{\sigma}^{2}(p t) / n t}{\hat{\sigma}^{2}(p)+\frac{\hat{\sigma}^{2}(p t)}{n_{i}}+\frac{\hat{\sigma}^{2}(r)}{n_{r}}+\frac{\hat{\sigma}^{2}(p r)}{n_{r}}+\frac{\hat{\sigma}^{2}(r t)}{n_{r}{ }^{*} n_{i}}+\frac{\hat{\sigma}^{2}(p r r, e)}{n_{r} n_{i}}}
$$

SDD between individual status scores: $\pm 1.96 * S E M * \sqrt{2}$

$$
\pm 1.96 * \sqrt{\frac{\hat{\sigma}^{2}(r)}{n_{r}}+\frac{\hat{\sigma}^{2}(p r)}{n_{r}}+\frac{\hat{\sigma}^{2}(r t)}{n_{r} * n_{j}}+\frac{\hat{\sigma}^{2}(p r t, e)}{n_{r} * n_{i}}} * \sqrt{2}
$$

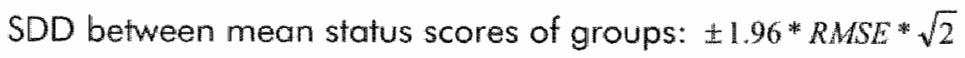

$$
\pm 1.96 * \sqrt{\frac{\hat{\sigma}^{2}(p)}{n_{p}}+\frac{\hat{\sigma}^{2}(p t)}{n_{p} * n_{\mathrm{r}}}+\frac{\hat{\sigma}^{2}(r)}{n_{r}}+\frac{\hat{\sigma}^{2}(p r)}{n_{p} * n_{r}}+\frac{\hat{\sigma}^{2}(r t)}{n_{r} * n_{r}}+\frac{\hat{\sigma}^{2}(p r t, e)}{n_{p} * n_{r} * n_{r}}} * \sqrt{2}
$$

\section{Explanation formulare}

When assessing the capacity of a radiological scoring method to detect differences between independently obtained status scores, the object of measurement is the variance caused by true differences between status scores of patients $\left(\hat{\sigma}^{2}(p)\right)$. In this study, the $2^{\text {nd }}$ film (after 1 year) is regarded as a $2^{\text {nd }}$ opportunity to assess these true differences in status. Thus, the variance in scores due to the facel "time" cannot be regarded as part of measurement error when assessing the reliability of the status scores. In other words the variance component of the facet "time" $\left\{\hat{\sigma}^{2}(t)\right)$ is a fixed facet and should not be included in the formula for SEM or RMSE. Consequently, the interaction $\left(\hat{\sigma}^{2}(p t)\right)$ becomes part of the variability in the patient score, the object of measurement. ${ }^{3.3}$ The variance components regarding the facet rater are all divided by $n$, which, makes it possible to evaluate the influence of the use of mean scores of raters on 
the statistics. In principle it is also possible to evoluate the influence of the use of mean scores of more than one time point by dividing the variance component regarding the facel time by $n_{i}$. In these reliability coefficients it is however not usefull to investigate this because the time period between the films of 1 year may cause the damage on the films to change. Therefore $n_{\text {; }}$ is in all formulae sef at 1 .

Sensitivity to change

\section{Formulae}

Responsiveness reliability coefficient:

$$
\frac{\hat{\sigma}^{2}(r) / n_{t}+\hat{\sigma}^{2}(p t) / n_{t}}{\frac{\hat{\sigma}^{2}(r)}{n_{t}}+\frac{\hat{\sigma}^{2}(p t)}{n_{t}}+\frac{\hat{\sigma}^{2}(r t)}{n_{r} * n_{r}}+\frac{\hat{\sigma}^{2}(p r t, e)}{n_{r} * n_{t}}}
$$

SDC of individual scores over time: $\pm 1.96 * S E M * \sqrt{2}$

$$
\pm 1.96 * \sqrt{\frac{\hat{\sigma}^{2}(r t)+\hat{\sigma}^{2}(p r r, e)}{n_{r} * n_{t}}} * \sqrt{2}
$$

SDC of mean group scores over time: $\pm 1.96 * R M S E * \sqrt{2}$

$$
\pm 1.96 * \sqrt{\frac{\hat{\sigma}^{2}(p t)}{n_{p} * n_{t}}+\frac{\hat{\sigma}^{2}(r)}{n_{p_{k}} * n_{t}}+\frac{\dot{\sigma}^{2}(p r i, e)}{n_{p} * n_{p} * n_{t}}} * \sqrt{2}
$$

\section{Explanation formulae}

The object of measurement in responsiveness statistics is the variance caused by true within-patient changes due to the elapse of time, reflected in our design by the variance component of the facet "time" $\left(\hat{\sigma}^{2}(r)\right)$. The variance component measurement error when astient variation $\left(\hat{\sigma}^{2}(p)\right)$ is here not part of the baseline levels are not the objessing changes in scores over time. In other words: change over time. This is abjects of measurement when assessing differences in baseline a covariate in ANCOVAus to considering between-patient variation at treatment effects in trials. The facet analyses or using change-scores to evaluate formula. This hold's for The facet "patient" is thus treated as fixed in the ICC individuals too. As a consequence, to calculate the SDCs over time for becomes part of the variability of the the "patient * time" interaction $\left(\hat{\sigma}^{2}(p r)\right)$ addition, the variance compon of the facer "fime", the object of measurement. In "patient * rater" $\left(\hat{\sigma}^{2}(p r)\right)$ are al the facet "rater" $\left(\hat{\sigma}^{2}(r)\right)$ and the interaction 
assessing changes over time. After all, these variance components reflect the variance in status scores due to the differences between the raters and do not influence changes in status scores. The influence of the differences between the raters that affects the changes in status scores is the "rater * time" interaction $\left(\hat{\sigma}^{2}(n t)\right)$. Consequently, the variance component of the "rater * time" interaction should be regarded as part of the SEM or RSME, but the variance component of the facet "rater" and the interaction "patient "rater" should not. When assessing the SDC over time an group level, the variance component of the interaction "patient * time" $\left(\hat{\sigma}^{2}(p t)\right)$ is included in the RMSE because this interaction reflects the variability of change between patients; this will be part of the error term when discriminating between mean group changes. Finally, the main facet "fime" $\left(\hat{\sigma}^{2}(t)\right)$ is not included in the RMSE to calculate the SDD of change over time on the group level. The reason is that our purpose is to assess the measurement error when evaluating change occurring over 12 months and not to generalize to all possible other time points. In other words, the facet "time" is fixed.

Alternative method to assess the sensitivity to change: using change-scores

When there are only 2 time points, the measurement error when assessing change over time can also be calculated directly from an ANOVA of the change-scores (the score obtained by subtracting the status score at time Ifrom the status score at time 21. The SDC over time is then assessed by calculating the $95 \%$ confidence interval around the change-scores with the formula \pm 1.96 * SEM. Notice that the SEM need not to be multiplied by $\sqrt{2}$ here because this SEM is already based on the change-score. This SDC over time assessed with the model using the change-score as single outcome measure should result in the same SDC over time assessed with the model above, using the status score als outcome measure. However note that simplifying the ANOVA by combining the variance components of the signal las happens when the facet "time" and the interaction "patient "fime" are combined into a facet "patient-change") may lead to loss of information potentially resulting in misinforming $1 \mathrm{CCs}$, as shown in the following example. In the extreme situation that all the patients's scores change enormously in the same way, the variance in change-scores is zero and this will result in a reliability coefficient based on the change-score of also zero. despite the large changes in scores. The responsiveness reliability coefficient as used in this study, however, won't be (close to) zero in such a situation: the variance component of the interaction "patient * time" $\left(\hat{\sigma}^{2}(p t)\right)$ will indeed also be zero, but the variance component of the facet "time" $\left(\hat{\sigma}^{2}(t)\right)$ will be wery large, leading to a large coefficient as expected and desired. 


\section{References}

1. Sharp JT, Young DY, Bluhm GB, Brook A, Brower AC, Corbett M, Decker IL, Genant $H K$, Gofton JP, Goodman N, ef al. How many joints in the hands and wrists should be included in a score of radiologic abnormalities used to assess theumatoid arthritis? Arthritis Rheum 1985;28:1326-35.

2. Rou R, Wassenberg $S$, Stucki $G$, Gebler-Ruckmann A. A new method of scoring radiographhic change in Rheumatoid Arthritis. J Rheumatol 1998;25:2094-2107.

3. wan der Heifde $D$. How to read radiographs according to the Sharp/wan der Heilde method. J Rheumatol 2000;27:261-3.

4. van der Heijde D, Dankert T, Nieman F, Rau R, Boers M. Reliability and sensitivity to change of a simplification of the Sharp/van der Heilde radiological assessment in rheumatoid arthritis. Rheumatolagy (Oxford) 1999;38:941-7.

5. Larsen A, Dale K, Eek M. Radiagraphic evaluation of theumatoid arthritis and related conditions by standard reference films. Acta Radiol 1977:18:4811-91.

6. Larsen A. How to apply Larsen scare in evaluating radiographs of Rheumatoid arthritis in longter, studies. J Rheumatol 1995;22:1974-5.

7. Scott DL, Houssien DA, Laasonen L. Proposed madification to Larsen's scoring methods for hand and wirist radiographs. Br J Rheumatol 1995;34:56.

8. Guyatt $G H$, Kirshner B, Jaeschke R. Measuring health status: what are the necessary measurement properties? J Clin Epidemiol 1992;45:1341-5.

9. Cuchacovich $M$, Couret $M$, Peray P, Gatica $H$, Sany J. Prescision of the Larsen and the Sharp methods of assessing radiologic change in patients with Rheumatoid Arthritis. Arthritis Rheum 1992;35:736-9.

10. Drossaers-Bakker KW, Amesz E, Zwinderman AH, Breedveld FC, Hazes JM. A comparison of three radiologic scoring systems for the long-term assessment of rheumatoid arthritis: findings of an ongoing prospective inception cohort study of 132 women followed up for a median of twelve years. Arthritis Rheum $2000 ; 43: 1465-72$.

11. Guth A, Coste J, Chagnon S, Lacombe P, Paolaggi J. Reliability of the three methods of radiologic assessment in patients with Rheumatoid Arthritis. Invest Radio $1995,30: 181-5$.

12. Lassere M, Boers $M$, van der Heijde D, Baonen A, Edmonds J, Saudan $A$, Verhoeven A. Smallest detectable difference in radiological progression. I Rheumatol

13. O Sullwan MM, Lewis PA, Newcombe RG, Broderick NJ, Robonson DA, Coles EC Jessop JD. Precision of larsen grading of radiographs in assesing progression of Rheumatoid anthritis in individual patients. Ann Rheum Dis 1990;49:286-9.

14. Paimela L, Laasonen L. Helve T. Comparison of the original and the modified Larsen methods and the Sharp method in scoring radiographic progression in early
Rheumatoid Arthritis. I Rheumatol 1998;25:1063*6. 
15. Pincus T, Larsen A, Brooks RH, Kaye J, Nance EP, Callahan LF. Comparison of 3 quantitative measures of hand radiographs in patients with theumatoid arthritis: Steinbracker stage, Kaye Modified Sharp score, and the Larsen score. J Rheumatol $1997: 24: 2106-22$.

16. Plant MP, Saklatvala J, Borg AA, Jones PW, Dawes PT. Measurement and prediction of radiological progression en early Rheumatoid Anthritis. J Rheumatol $1994: 21: 1808-13$.

17. Rau $R$, Herborn $G$. Development and evaluation of a modified version of the Larsen method for evaluating rontgenlogic changes in chronic polyarthritis. $Z$ Rheumatal $1997: 56: 127-35$.

18. Sharp JT, Bluhm GB, Brook A, Brower AC, Corbett $M$, Decker $J L$, Genant $H K$, Gofton JP, Goodman N, Larsen A, ef al. Reproducibility of multiple-observer scoring of radiologic abnormalities in the hands and wrists of patients with rheunatoid arthritis. Arthritis Rheum 1985;28:16-24.

19. Sharp IT. Radiologic assessment as an outcome measure in rheumatoid arthritis. Arthritis Rheurm 1989;32:221-9.

20. van der Heijde $D$, Boonen $A$, Boers $M$, Kostense $P$, van der Linden $S$. Reading radiographs in chronological order, in pairs or as single fitms has important implications for the discriminative power of rheumatoid arthritis clinicall trials. Rheumatology (Oxford) 1999;38:1213-20.

21. Wassenberg s, Herborn G. Comparison of Larsen's and Sharp's method of scoring radiographs in Rheumatoid arthritis. Arthritis Rheum 1994;5upplement of $1994: 5250$.

22. Stratford PW, Goldsmith CH. Use of the standard error as a reliability index of interest: an applied example using elbow flexor strength data. Phys Ther $1997: 77: 745-50$.

23. Wynwich KW, Nienaber NA, Tierney WM, Wollinsky FD. Linking clinical relewance and statistical significance in evaluating intra-individual changes in health-related quality of life. Med Care 1999:37:469-78.

24. Ladak HM, Thomas JB, Mitchell JR, Rutt BK, Steinman DA. A semi-automatic technique for measurement of arterial wall from black blood MRI. Med Phys $2001: 28: 1098-107$.

25. Odom CJ, Taylor AB, Hurd CE, Denegar CR. Measurement of scapular asymetry and assessment of shoulder dysfunction using the Lateral Scapular Slide Test: a reliablity and validity study. Phys Ther 2001;81:799-809.

26. Roebroeck ME, Harlaar J, Lankhorst GJ. The application of generalizability theory to reliability assessment: an illustration using isometric force measurements. Phys Ther $1993 ; 73: 386-95$

27. Geertzen JH, Dijkstra PU, Stewart RE, Groothoff JW, Ten Duis HJ, Eisma WH. Variation in measurements of range of motion: a study in reflex sympathetic dystrophy patients. Clin Rehabil 1998;12:254-64.

28. Beckerman $H$, Roebroeck ME, Lankhorst GJ, Becher JG, Bezemer PD, Verbeek AL. Smallest real difference, a link between reproducibility and responsiveness. Qual Life Res 2001; 10:571-8.

29. Guyatt $G$, Walter $S$, Norman $G$. Measuring change over lime: assesing the usefulness of ewaluation instruments. J Chron Dis 1987;40:171-8. 
30. Kozis LE, Anderson JJ, Meenan RF. Effed sizes for interpreting changes in health status. Med Care 1989:27:S178.89.

31. Liang MH, Fossel AH, Larson MG. Comparisons of five health status instruments for onthopedic evaluation. Med Care 1990;28:632-42.

32. Husted JA, Cook Ru; Farewell VT, Glodman DD. Methods for assessing responsiveness: a crifical review and recommendations. I Clin Epidemia 2000,53:459-68.

33. Streiner DL. Norman GR. Generalizability theory. Health measurements scales. A practical guide to their development and use.: Oxford University Press, 1995: $128-743$.

34. Shrout P, Fleis J. Intraciass correlations: use in assessing rater reliability. Psychal Bull 1979;86:420-8.

35. Crambach $L$, Gleser $G$, Nanda $H$, Rajaratnam N. The dependability of behavianal measurements: Theory of generalizability of scores and profiles. New York: Wiley, 1972.

36. Cronbach $L$, Rajaratnam $N$, Gleser $G$. Theory of generalizability: A liberalitization of reliability theory. Br $\$ Stat Psychol 1963;16:137-63.

37. Shavelson R, Webb N, Rowley G. Generalizability theary. American psychologist 1989;44:922-32.

38. Shavelson RJ, Wenn NM. Generalizability theory: a primer. Newbury Park London New Delhi: SAGE publications, 1991.

39. Lassere $M N$, van der Heijde $D$, Johnson $K$, Bruynesteyn $K_{*}$ Molenaar $E_{\text {; }}$ Boonen $A$, Verhoeven A, Emery P, Boers M. Robustness and generalizability of smallest detectable difference in radiological progression. J Rheumatol $2001 \cdot 28: 91$ 1 -3 .

40. Bruynesteyn $K$, van der Heïjde $D$, Boers $M$, Verhoeven $A$, Boonen $A_{\text {, wan }}$ der Linden $S$. Contribution of progression of erosive damage in previously eroded joints in early rheumatoid arthritis trials: COBRA trial os an example. Arthritis Rheum $2002 ; 47: 532-6$.

41. Edmonis 1, Saudan A, Lassere $M$, Scoff $D$. Introduction to reading radiographs by the Scott modification of the Larsen method. J Rheumatol 1999;26:740.2.

42. Bruynesteyn $K$, wan der Heipde D, Boers $M$, Savdan $A$, Peloso Pa, Paulus H, Houben $H$. Griffiths B, Edmonds J, Bresnihon B, Boonen $A$, Van Der Linden S. Detecting radiological changes in theumatoid arthritis that are considered important by clinical experts: influence of reading with or without known sequence. I Rheumatol 2002:29:2306-12

43. Boers M, Verhoeven AC, Markusse HM, van de Laar M, Westhovens $R$, wan Denderen $J C$, van Zeben D, Dijkmans BA, Peeters AJ, Jacobs $P$, van den Brink HR, Schouten HJ, wan der Heijde DM, Boonen A, wan der Linden S. Randomised comparison of combined step-down prednisolone, methotrexate and sulphasalazine with sulphasalazine alone in early theumatoid arthritis Lancel 1997;350:309-18.

44. Armett FC, Edwarthy SM, Bloch DA, MCShone DJ, Fries JF, Cooper NS, Healey LA, Kaplan SR, Liang MH, Luthra HS, el al. The American Rheumatism Assaciation 1987 revised criteria for the classification of rheumatoid arthritis. Arthritis Rheum
$1988: 37: 315-24$.

45. Nocera FD, Ferlazzo F, Borghi V. G theory and the reliability of psychophysialogical measures: a tutorial. Psychophysiology $2001 ; 38: 796-806$. 
46. Crick GE, Brennan RL. GENOVA: a fortran 77 program for analysis of variance and generalizability analyses with balanced designs. lowa City, lowa: The American College Testing Program, 1984.

47. McNemar Q. Psychalogical statistics. New York: Wiley \& Sons, 1969:154-87.

48. Neter J, Wasserman $W$, Kunner MH. Applied linecir statistical models: regression, analyses of variance and expirimental designs. Homewood, IL: R.D. Irwan, 1990.

49. Guyalt GH, Juniper EF, Walter SD, Griffith LE, Goldstein RS. Interpreting treatment effects in randomised trials. BMI 1998,316:690-3.

50. Lipsky PE, van der Heijde DM, St Clair EW, Furst DE, Breedveld FC, Kalden IR, Smolen JS, Weisman $M$, Emery P, Feldmann $M$, Harriman $G R$, Maini RN; AntiTumor Necrosis Factor Trial in Rheumatoid Arthritis with Concomitant Therapy Study Group. Infliximab and methotrexate in the treatment of rheumatoid anthritis. AntiTumor Necrosis Factor Trial in Rheumatoid Arthritis with Concomitant Therapy Study Group. N Engl I Med 2000;343:1594-602.

51. van der Heije DM, van Riel PL, Nuwer-Zwant IH, wan de Putte LB. Alternative methads for analysis of radiographic damage in a randomized, double blind, parallel group clinical trial comparing hydroxychloroquine and sulfasalazine. I Rheumatol 2000;27:535-9.

52. Scott DL, Pugner K, Kaarela K, Doyle DV, Woolf A, Holmes J, Hieke K. The links between joint damage and disability in rheumatoid arthritis. Rheumatology (Oxford) 2000,39:122-32.

53. Bruynesteyn $K$, van Der Heïjde D, Boers $M$, Saudan $A$, Peloso Pr Paulus $H_{s}$ Houben H, Griffiths B, Edmonds J, Bresnihan B, Boonen A, van der Linden S. Determination of the minimal clinically important difference in rheumataid anthritis joint damage of the Sharp/van der Heijde and Larsen/Scott scoring methods by clinical expents and comparison with the smallest detectable difference. Arthritis Rheum 2002;46: $913-20$.

54. Brennan RL. (Mis)conceptions about generalizability theory. Educational Mecsurement: Issues and Practice 2000;19:5-10.

55. Fries JF, Bloch DA, Sharp JT, McShane DJ, Spitz P, Bluhm GB, D, Genant H, Gofton $P$, Richman $S$, et al. Assessment of radiologic progression in rheumatoid arthritis. A randomized, controlled trial. Arthritis Rheum 1986;29:1.9.

56. Beaion DE, Bombardier C, Katz JN, Wright JG. A taxonomy for responsiveness. J Clin Epidemiol 2001:54:1204-17.

57. Atkinson G, Nevill AM. Statistical methods for assessing measurement error (reliability) in variables relevant to sports medicine. Sports Med 1998;26:217-38.

58. Bland JM, Altman DG. Statistical methods for assessing agreement between two methods of dinical measurement. Lancet 1986;1:307-10. 


\section{Chapter 3}

Contribution of progression of erosive damage in previously eroded joints in early rheumatoid arthritis trials: COBRA trial as an example for the COBRA trial group

Arthritis Rheum 2002;47:532-6 


\section{Abstract}

\section{Aim}

In meumatoid arthritis (RA) in the context of a drug trial, prevention of erasions in undamaged joints is offen considered more important than prevention of progression in already damaged joints, although a clear rationale is lacking. The aim of this study is to ewaluate the relative contribution of separate components of the erosion score of the madified Sharp/wan der Heijde method in early RA.

\section{Methods}

Different aspects of erosive damage were evaluated by their ability to discriminate between the 2 treatments in on early RA trial the COBRA trial).

\section{Results}

The contribution of progression of already eroded joints to the total erosion score clearly increased during the 1.5 years of the trial. When the periods $0-28,28-56$ and 56-80 weeks were analyzed separately, the erosion score showed a significant difference between the groups in the first 2 periods $(P<0.0001, P=0.03$, and $P=0.64$, respectively). Similar differences were seen in rates of progression in previously eroded joints. $(P=0.005, P=0.003, P=0.35)$. On the other hand, rates af progression in newly eroded joints showed no significont difference between the 2 treatment groups in the second and third period $(P<0.0001, P=0.16, P=0.87)$. Analyses on joint and patient level showed analogous results.

\section{Conclusion}

Subanalyses on progression rates in noneroded joints and already eraded joints can provide additional information. However, important information and discriminative strength may be lost when assessment is limited to the development of erosions in undarnaged joints. 


\section{Introduction}

Persistent arthritis caused by the chronic inflammatory disease rheumatoid arthritis (RA) often leads to joint damage, which can be visualized on radiographs. The most specific features of RA are erosions and joint space narrowing. Prevention of erosions in undamaged joints is often considered to be more important than prevention of progression in already damaged joints, especially in early RA patients, although this has never been thoroughly validated. In a recent cohort of RA patients followed during the first 6 years of disease, progression of previously existing damage proved as important as new abnormalities in previously undamaged joints. 'The aim of the present study was to evaluate the weight of the different components of erosive joint damage and their discriminative ability in a clinical trial among patients with early RA.

\section{Patients and Methods}

Radiographs from the COBRA trial were used in this study. The COBRA trial is a double blind, randomized controlled trial, with 2 active treatment arms (combined therapy versus monotherapy). This study showed less progression of radiographic joint damage in patients taking combined treatment with stepdown prednisolone, methotrexate and sulfasalazine compared with treatment with sulfasalazine alone. The 56-week clinical trial included 155 patients with early RA, aged 23-70 years, who fulfilled the American College of Rheumatology (formerly American Rheumatism Association) 1987 classification criteria for RA. ${ }^{2}$ Radiographs, taken ot baseline and at weeks 28,56 and 80 were scored according to the modified Sharp/van der Heijde method. ${ }^{3}$ The films were scored independently by 2 observers, each blinded for patient and treatment. The chronological sequence of the films was known by the observers. The trial has been published in detail elsewhere. ${ }^{4}$

The Sharp/van der Heijde score assesses erosions and joint space narrowing separately. Erosion score ranges from 0 to 280, with a maximum of 160 in the hands and 120 in the feet. Erosions are scored 1 if they are discrete and 2 or 3 depending on the surface area of the joint involved, with a maximum of 5 per joint in the hands and 10 per joint in the feet. The different aspects of progression of erosive damage and the ability of these sub-scores (Table 3.1) to discriminate between the 2 active treatments were compared with the original Sharp /van der Heijde erosion progression score. Along with the progression rate of the erosion score in noneraded joints and the progression rate of the erosion score in eroded joints, we also looked at the appearance of erosions without taking into account the size of the erosions, i.e., the number of erosions. Progression rate refers to the increase of that specific feature in the specific time periad studied. Progression scores were based on the count or score of erosions, 
not on the count of joints. Count af joints with progression of erosive damage was evaluated in separate analyses for joints with and without previous erosions. Finally, the numbers of patients with progression of erosive damage in previously noneroded and eroded joints were analyzed (analyses on patient levell.

TABLE 3.1.

Description of progression scores

\begin{tabular}{ll} 
Nomenclature & Description \\
\hline Erosion score & Number of erosions in noneroded joints \\
& Number of new erosions in eroded joints \\
& Weighting for erosion size \\
Number of erosions & Number of erosions in noneroded joints \\
Erosion score, noneroded joints & Number of new erosions in eroded joints \\
Erosion score, eroded joints & Wumber of erosions in noneroded joints \\
& Number of new erosions in eroded joints \\
& Weighting for erosion size
\end{tabular}

Statistical analysis

Patients were included in the analyses if they had radiographs at baseline and at least one followup. Differences in median changes between the treatment groups were analyzed with Mann-Whitney rank sum tests, because of the nonnormal distribution of the data. Differences in percentage of joints were analyzed with chi-square tests. All tests were 2-tailed, and $P$ values of $<0.05$ were considered significant.

\section{Results}

Figure 3.1 shows the progression rates of the erosion score and the subscores in each treatment group per study period. Within the first 28 weeks, the median progression rate of erosive damage in the 2 treatment groups differed significantly regardless of the score used (erosion score for all joints, $\mathrm{P}<0.0001$;
number of erosions, $\mathrm{P}<0.0001$; erosion score for noneroded joints, $\mathrm{P}<0.0001$;
erosion score for eroded joints, $\mathrm{P}=0.005$ ). erosion score for eroded joints, $\mathrm{P}=0.005$ ). In the second period (week 28-56) the median progression rate of the erosion score was 1.5 (range $0-17.5$ ) in the
sulfasalazine group versus 0.5 (range $0-26$ ) in the combined treatment group,
resulting in a $P$ value of 0.03 when testing the resulting in a $\mathrm{P}$ value of 0.03 when testing the differences between the 2 treatment groups with the Mann-Whitney rank sum test. The progression in number of erosions also was statistically significantly different between the 2 
groups $(P=0.02)$. However, the progression rate of the erosions score in noneroded joints showed no significant difference $(P=0.16)$ between the 2 groups during this period; the median increase in erosion score in non-eroded joints was 0.5 (range 0-8.5) in the sulfasallazine group and also 0.5 (range 0 $15)$ in the combined-treatment group. On the other hand, the 2 groups differed significantly in the progression rate in already eroded joints $(P=0.003)$; the median increase in score in eroded joints was 1.0 (range $0-13$ ) in the sulfasalazine group and 0.0 (range $0-11$ ) in the combined treatment group. During weeks 56-80, when both patient groups were treated with sulfasalazine only, no aspect of the progression of erosive damage differed significantly between the groups (erosion score for all joints, $\mathrm{P}=0.64$; number of erosions, $\mathrm{P}=0.52$; erosion score for noneroded joints, $\mathrm{P}=0.87$; erosions core for eroded joints, $P=0.35$ ).
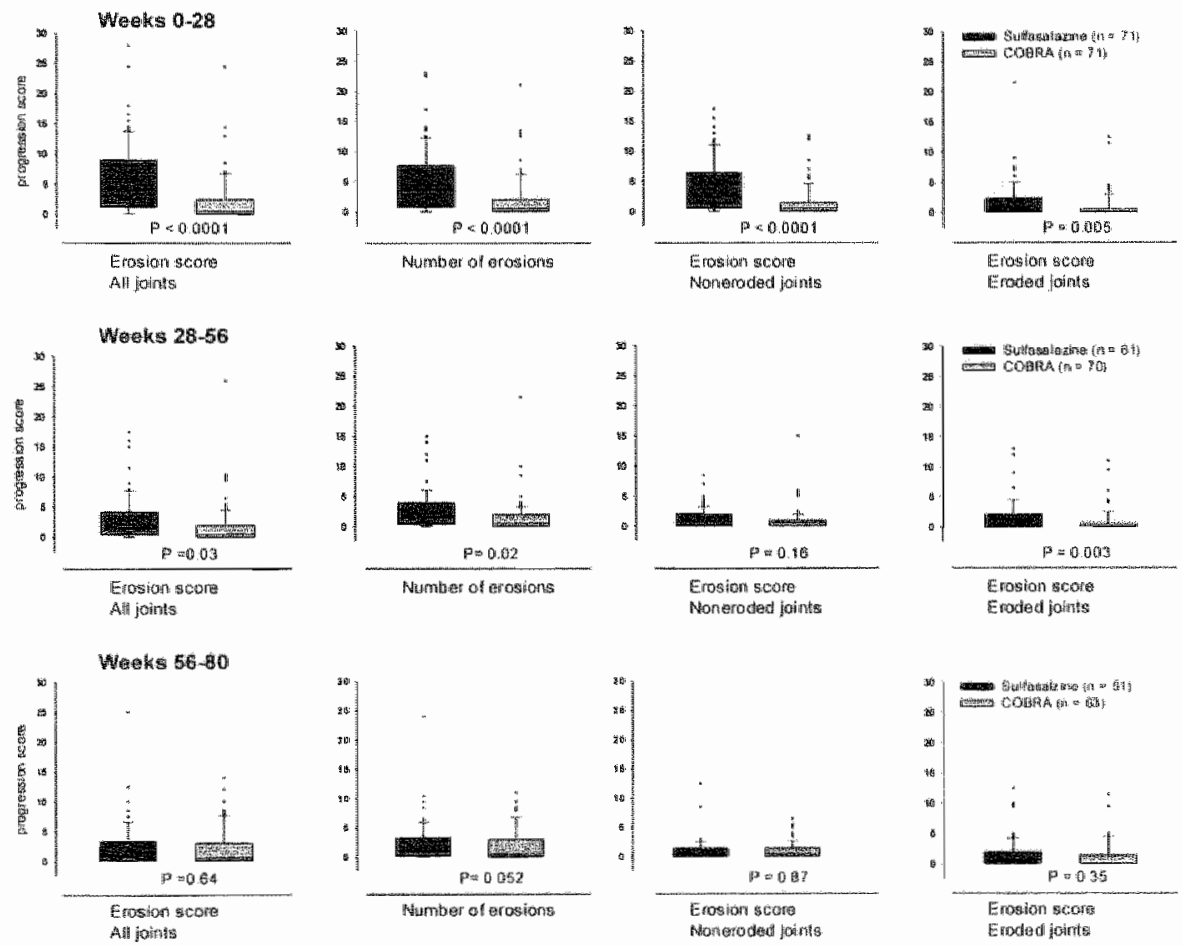

FIGURE 3.1.

Progression rates of the erosion score and its components per study period: Box-whisker plots: horizontal line in box = median; dotted line in box = mean; limits of box $=25$ th and 75 th percentiles; whiskers $=10$ th and 90 percentiles; asterisks = outliers; COBRA $=$ acronym for Combination therapy in RA; $p$-values (beneath boxes) calculated with MannWhitney rank sum tests comparing the 2 treatment groups for that specific score 
The importance of progression of previously eroded joints and the decreasing rate of erosians in noneroded joints over time is demonstrated in Figure 3. 1. The contribution of the progression rate of the erosion score in eraded joinis to the total erosion score exceeded that of the erosion score in noneroded joints in the period 56.80 week period.

We also studied the different progression rates of the $0-56$ and $0-80$ week periods. Compared to the analysis of the 28-56 week period, the pragression rate of already eroded joints in the 0.56 week period was of minor importance and not discriminative $(P=0.07)$. The reason for this is that there were only a few already eroded joints at baseline to contribute to this progression rate (i.e., median 3.0 joints in the sulfasalazine group and 2.0 joints in the combinationtherapy group). Moreover, the period in which new joints can develop erosions is now twice as long: the erosion score in noneroded joints now includes the progression of erosions in the second period of 28 weeks of joints that developed erosions in the first 28 weeks. As a consequence, assessing the rate of progression of the total erosion score, the numbers of erosions or the erosion score in noneroded joints yielded similar information: each method discriminated equally between the groups, resulting in $P$ values of $0.002,0.001$ and 0.001 respectively. The same was found when evaluating the entire study period $(0.80$ weeks), resulting in $P$ values of $0.005,0.004$, and 0.003 respectively.

Analyses on joint level showed similar results (Figure 3.2). Within the first 28 weeks, the number of joints with new erosions differed significantly between the 2 treatment groups in both eroded and noneroded joints $(P<0.0001$ and $P=0.02)$, the first being more discriminative than the latter. In the second period (week 28-56), previously eroded joints in the sulfasalazine group developed more new erosions than those in the combination-therapy group (median 1.0 and 0,0 respectively; $P=0.003$ ). However, the number of noneroded joints with new erosions did not differ between the 2 treatment groups (median for each 1.0 ; $P=0.13$ ) during this period, which is in accordance with the analyses of the
scores.

Table 3.2 shows the percentage of patients with new erosions, with newly eroded joints and those with progression of the erosive damage in already eroded joints. Contrary to the analyses on the scores and those on joint level, no statistically significant difference between 2 groups was found when analyzing all joints in period $28-56$ week $(75 \%$ versus $63 \%$ patients with new erosions; $\mathrm{P}=0.18$ ). On the other hand the treatment groups did again differ statistically significant when comparing the patients, with progression in already eroded joints $164 \%$ versus $41 \%$ patients with progression in already eroded joints; who developed new erosions were a new erosions and the number of patients weeks. As in the analyses on there also evaluated for the periods $0-56$ and $0-80$ joints did not add useful information when bores, analyses of already eroded the very low number of damaged when baseline was included, because of patient level also illustrate the joints at the beginning. The analyses on a significant differences if using a dichotomized outcome variable. 
Weeks 0-28

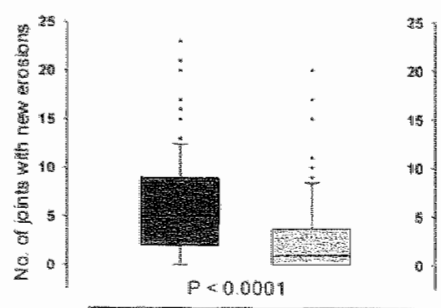

All joirtis

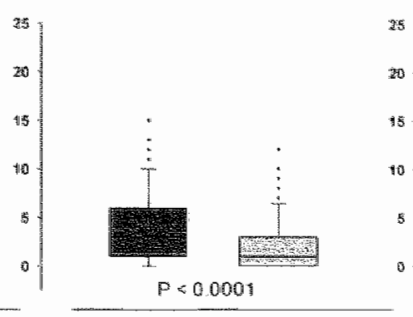

Nonerodect joints

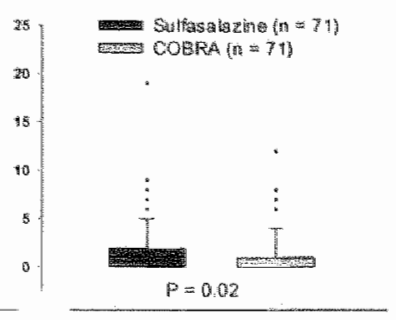

Eroded joints

Weeks 28-56

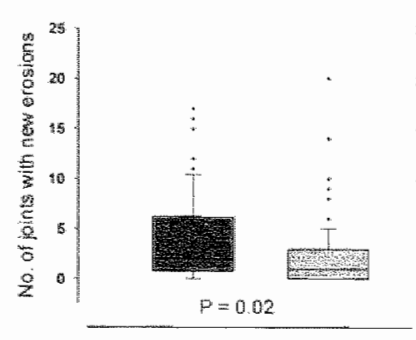

All joints

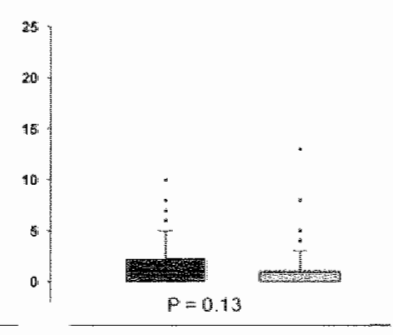

Noneroded joints

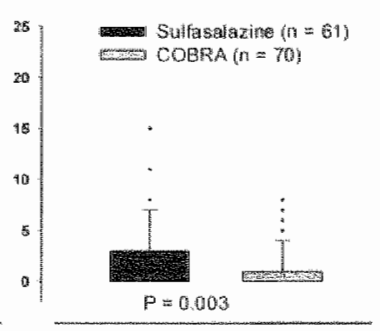

Eroded joints

\section{Weris $56-80$}

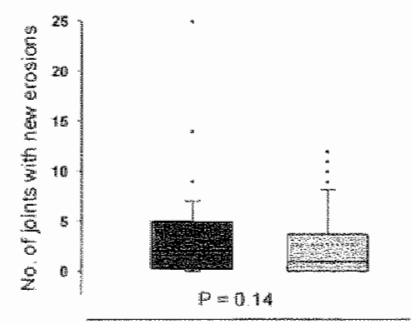

Aill bonts

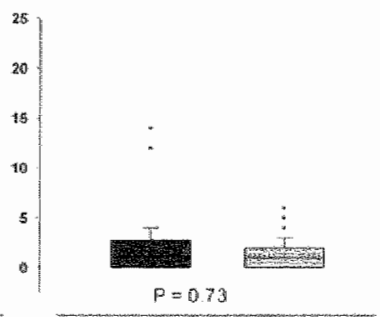

Noneroded joints

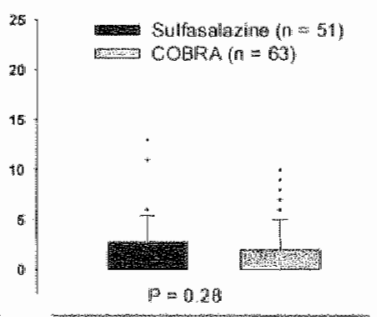

Eroded joints

\section{FIGURE 3.2.}

Number of joints with new erosions per study period icalculated in all joints, in previously non-eroded joints and previously eroded joints): Box-whisker plots: horizontal line in box = median; dotted line in box $=$ mean; limits of box $=25$ th and 75 th percentiles; whiskers $=10$ th and 90 percentiles; asterisks $=$ outliers; $\operatorname{COBRA}=$ acronym for Combination therapy in RA; p-values (beneath boxes) calculated with Mann-Whitney rank sum tests comparing the number of joints between the 2 treatment groups 
TABLE 3.2.

Percentage of patients with new erosions, newly eraded joints and progression of erosive damage in already eroded joints, according to treatment group and study period.

\begin{tabular}{|c|c|c|c|}
\hline 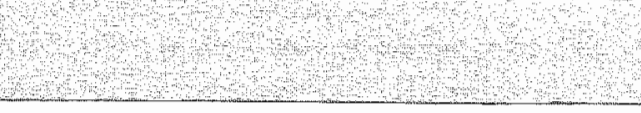 & $\begin{array}{c}S S Z \\
\%\end{array}$ & $\begin{array}{c}\text { COBRA } \\
\%\end{array}$ & Pt \\
\hline \multicolumn{4}{|l|}{ Period 0.28} \\
\hline Patients with new erosions & 83 & 65 & 0.02 \\
\hline Patuents with newly eroded joints & 78 & 51 & 0.002 \\
\hline Patients with progression in eroded joints & 56 & 34 & 0.07 \\
\hline \multicolumn{4}{|l|}{ Period $28-56$} \\
\hline Patients with new erosions & 75 & 63 & 0.18 \\
\hline Potients with newly eroded joints & 57 & 51 & 0.62 \\
\hline Patients with progression in eroded joints & 64 & 41 & 0.02 \\
\hline \multicolumn{4}{|l|}{ Period $56-80$} \\
\hline Patients with new erosions & 75 & 73 & 0.97 \\
\hline Patients with newly eroded joints & 59 & 64 & 0.76 \\
\hline Potients with progression in eroded joints & 51 & 38 & 0.20 \\
\hline
\end{tabular}

\section{Discussion}

Our analyses assessed the contribution of separate components of erosive

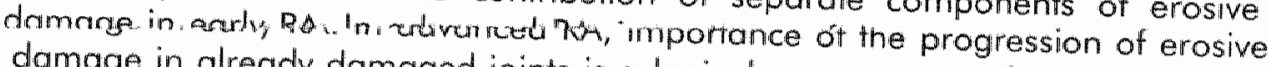
damage in already damaged joints is a logical consequence of the decrease in the number of undamaged joints. Our results showed that also in patients with early RA, subanalyses of already eroded joints can add important information in the evaluation of treatment efficacy. The effect, however, was only apparent and relevant when the assessment period did not include baseline, as a result of very low number of damaged joints at baseline.

The contribution of erosive damage in eroded joints to the total erosion score increased over time: In the period 56-80 weeks, damage in eroded joints added even more than did progression in noneroded points. In addition, no statically significant difference was found between the treatment groups when comparing the progression rates of the erosion scores in noneroded joints in the second half-year of the study. However, when comparing the progression rates in eroded joints there was a statically significant difference. The same results were found when analyzing on joint or patient level.

Assessing the number of new erosions did discriminate to the same extent as the total erosion score (including weighting of the erosions). However, in advanced 
disease discriminative strength might be diminished by not taking into account the size of erosions.

Although the joint space-narrowing subscore has received no attention in this study, there is every reason to assume the described effects are also present in non-narrowed or nondamaged (i.e., previously undamaged joint developing an erosion or joint space narrowing) joints. Assessing joint space narrowing adds relevant information, and is also relevant for the study of pathogenesis. Subscores remain relevant for pathogenic purposes.

This study analyzed data from the COBRA trial, published in detail earlier. ${ }^{4}$ Recent analyses showed that even after 4 to 5 years the initial 6-month combination therapy with high-dose conticosteroids resulted in sustained suppression of the radiographic progression rate. ${ }^{5}$

In conclusion, because current trials have only moderate power to defect differences in progression, it is important to apply the most sensitive measure. In the case of erosions, that means using information on number and weight of erosions regardless of the previous state of joints. Subanalyses can be done in addition to provide extra information on treatment efficacy. 


\section{References}

1. Hulsmans $H M$, Jacobs $W_{\text {, }}$ van der Heijde DM, wan Albada-Kuipers GA, Schenk $Y$, Bijtsma JW. The course of radiologic damage during the first six years of rheumatoid arthritis. Arthritis Rheum 2000; 43: 1927-40.

2. Arnett FC, Edworthy SM, Bloch DA, MC Shane DJ, Fries JF, Cooper NS, Healey LA, Kaplan $\mathrm{SR}_{\text {s }}$ Liang $\mathrm{MH}$, Luthra $\mathrm{HS}$, ef al. The American Rheumatism Association revised criterio for the classification of rheumatoid arthritis. Arthritis Rheum $1988 ; 31: 315-24$

3. Van der Heijde D. How to read radiographs according to the Sharp/van der Heijde method. J Rheumatol 2000; $27: 261-3$.

4. Boers $M$, Verhoeven $A C$, Markusse $H M$, van de Laar $M$, Westhovens $R$, van Denderen JC, wan Zeben D, Dijkmans BA, Peeters AJ, Jacobs $\mathrm{P}_{\text {s }}$ wan den Brink HR, Schouten Hy, van der Heijde DM, Boonen A, van der Linden S. Randomised comparison of combined step-down prednisolone, methotrexate and sulphasalazine with sulphasalazine alone in early rheumatoid arthritis. Lancet 1997; 350: 309-18.

5. Landewé RBM, Boers M, Verhoeven AC, Westhovens $R$, wan de Laar MAFJ, Markusse $H M$ et al. COBRA Combination therapy in patients with early rheumatoid arthritis: long-term structural benefits of a brief intervention. Arthritis Rheum 2002;46:347-56 


\section{Chapter 4}

Determination of the minimal clinically important difference in rheumatoid arthritis joint damage of the Sharp/van der Heijde and Larsen/Scott scoring methods by clinical experts and comparison with the smallest detectable difference

Bruynesteyn K, van der Heijde D, Boers M, Saudan A, Peloso P, Poulus H. Houben H. Griffiths P. Edmonds d, Bresnihan $B$, Boonen $A$, van der Linden $S ;$ 


\section{Abstract}

\section{Aim}

To assess the mirimal clinically important difference (MCID) in joint damage on hand and foot radiographs of patients with early rheumatoid arthritis (RA) as assessed with the Sharp/van der Heijde and Larsen/Scot methods, and to study how the smallest detectable difference (SDD) relates to the MCID for each method.

\section{Methods}

The judgments of an international panel of expens on the dinical relevance of progression of joint damage as seen on sets of radiographs obtained at 1 -year intervals in 4 clinical settings (early wersus late RA and mild versus high disease activify) were used as the external criterion, which was compared with progression scores as determined by the 2 scoring methods. Progression scores with the highest combined sensitivity and specificity for detecting dinically relevant progression represented the MCID. Subsequently, the sensitivity and specificity of the scoring methods were determined when using the SDD as the threshold for relevant progression, and these were compared with the sensitivity and specificity of the MCID.

\section{Results}

The panel judged changes in joint damage around the level of the SDD (5.0) of the Sharp/won der Heijde method as minimal clinically important, resulting in satisfactory sensitivity (mean 79\%) and specificity (mean $84 \%$ for detecting clinically important progression in the 4 dinical settings when using the SDD as threshold value. The MCID (mean 2.3) of the Larsen/Scoft method was much smaller than its SDD (5.8) and the sensitivity for detecting dinically important progression by applying the SDD as threshold was consequently low (mean $51 \%$ ), accompanied with high specificity (mean $59 \%$ ).

\section{Conclusion}

This study suggests that the SDD of the Sharp/van der Heijde method can be used as MCID, i.e., as thresthold level for individual response criteria. The SDD of the Larsen/Scatt method, however, turned out to be too insensitive to use as threshold for individual climically relevart change. 


\section{Introduction}

Radiological joint damage is part of the core set of outcomes used to evaluate the disease modifying effects of therapies for rheumatoid arthritis (RA) in clinical trials that have duration of $\geq 1$ year.' When reparting the results of a trial, progression of radiologic joint damage, similar to most other outcome measures, is presented mainly on a group level, although change in damage occurring at the individual level can provide important additional information and may be easier to understand. ${ }^{2}$

To present data on an individual level, or in other words, to determine the number of patients actually showing a response to a particular drug, a threshold for clinically important response is necessary. Several strategies to define the socalled minimal clinically important difference $(M C I D)$ were presented and discussed at the fifth Outcome Measures in Rheumatology Clinical Trials (OMERACT V) conference. ${ }^{3-5}$ First, the threshold can be assessed through statistical analyses. In particular, the smallest detectable difference (SDD) is often used as the threshold level for the relevant progression of joint damage. ${ }^{6-8}$ The SDD represents the smallest change score that can be reliably discriminated from the measurement error of the scoring method. Using the SDD as the threshold ensures that the changes observed are real changes and not caused by random variability. Because the SDD is based on statistical analyses only, it is not known to what degree it represents a clinically relevant change. The second approach, the opinion-based model, actually assesses the clinical relevance of threshold levels, using the experience and knowledge of seasoned clinicians. The third and final approach defines the threshold on the ability of radiographic change scores to predict future outcome measures, such as disability or joint replacement, and is called data-driven or predictive approach.

An opinion-based approach, that is, determining the amount of progression of joint damage that would lead clinical experts to change their therapy, seemed to be a valid and logical step, after determining the SDD, in the assessment of the MCID in radiological joint damage caused by RA. At OMERACT $V$, preliminary results, abtained from a panel of 3 experts were presented and was indeed accepted as the next step towards defining this MCID and evaluating the statistically derived SDD., Because further validation of this approach was considered necessary, this article presents the final results from a panel of 5 experts.

The first aim of this study was to assess the MCID in joint damage among hand and foot radiographs obtained at 1 -year intervals, as determined by 2 of the most widely used radiographic scoring methods: the Sharp/van der Heilde method" ${ }^{11}$ and the Larsen/Scott method. ${ }^{12}$ Second, we studied the relationship between these MCID and the SDD and how the Sharp/van der Heijde and Larsen/Scott methods relate to each other concerning these matters. 


\section{Patients and Methods}

The judgment of an international panel of experts on the clinical relevance of the progression of joint damage seen on sets of radiographs obtained at 1-year intervals was used as the external criterion and was compared with the progression scores obtained by the Sharp/wan der Heijde and Larsen/Scott methods.

\section{Expert panel}

The expert panel consisted of 5 rheumatologists $(B B, B G, H H, H P$ and PP) who independently evaluated 46 pairs of hand and foot films, which had been obtained at 1 -year intervals from patients with early RA. The experts were first asked whether they noticed any progression of joint damage due to RA between the 2 sets of hand and foot films for each pair of films. Second, if they noticed progression, they had to state whether they considered that difference in joint damage to be clinically relevant in the following 4 clinical settings: 1) disease duration of 2 years and mild disease activity, 2) disease duration of 2 years and high disease activity, 3) disease duration of 8 years and mild disease activity and 4) disease duration of 8 years and high disease activity).

A total of 5 results were obtained simultaneously: 1 on the presence of progression and 4 on the clinical relevance of this progression for the 4 different settings. The settings were all based on a "typical RA patient": a 46-year-old female patient with RA treated with methotrexate for 1 year. Clinically relevant progression of joint damage was defined as progression that would make a clinician change the second line therapy.

Films were presented to the panel members in correct chronologic order, corresponding to routine clinical practice. The majority opinion of the panel (concordance among 3,4 or 5 of 5 members) was the criterion applied in all analyses. All radiographs were viewed twice by each panel expert, at an interval of at least 4 weeks, to estimate the intraobserver reliability. The order in which the sets of films for each patient were presented was different in the 2 viewing sessions. Unless stated otherwise, the opinion of the first viewing session was used in the analyses.

\section{Radiographic scoring methods}

Radiographs were scored independently according to the Sharp/van der Heiide 11 and Larsen/Scatt methods ${ }^{12}$, each conducted by 2 experienced readers $(D v H+A B$ and JE+AS, respectively). Films were scored in correct chronological order and the patient's identity was masked. The Sharp/van der Heilide method assesses erosions and joint space narrowing separately and has a range from 0 to 448 . This method was applied with the rule that scores cannot decrease by definition. ${ }^{13}$ The Larsen/Scott method has a range from 0 to 200 (total score of hands and feet) and applies 1 grade to each joint. The wrists are evaluated as 
single joints and are weighted by a facior 5. Mean scores of each pair of observers were used for further analyses.

\section{Patients and radiographs}

The film sets of a recent study an the precision and sensitivity to change of the Sharp/van der Heijde were used in this study. ${ }^{14}$ The 46 pairs of posteriaanterior hand and anteriorposterior foot films were oblained from 22 patients. The films were selected in the previous study for high and low baseline scores and for high and low progression scores between the 2 sets of 1 pair of films. All patients fulfilled the American College of Rheumatology (formerly, American Rheumatism Association) 1987 classification criteria for $\mathrm{RA}^{15}$ and had a disease duration of $<1$ year at the start. Ten patients had had a followup period of 1 year and supplied 1 pair of films each, while 12 patients had had a followup period of 3 years and supplied 3 pairs each.

\section{Statistical analyses}

Receiver operating characteristic (ROC) curve analyses evaluated the sensitivity and specificity of the scoring methods for discriminating between clinically relevant progression and no progression as defined by the expert panel. A ROC curve plots the true-positive rate (sensitivity) as a function of the false-positive rate (100-specificity) at all possible threshold levels. ${ }^{16}$ In other words, the sensitivity and specificity for detecting a clinically important change was determined for every progression score, done separately for all settings. The progression score with the highest accuracy i.e., highest sensitivity and specificity combination, represented the MCID in that particular setting. In the ROC curve, this is the point nearest the upper left corner, where the sensitivity and specificity is $100 \%$. Figure 4.1 is an example of a ROC curve. Note that the progression score that discriminated best between clinically relevant progression and no progression, as defined by the panel, represented the MCID and not the lowest progression score that was judged clinically relevant because the latter would be $100 \%$ sensitive but not specific at all.

Further, the sensitivity and specificity of the scoring methods were calculated with the SDD, as determined by 2 observers, as threshold for relevant progression. The SDD is a statistical method for defining measurement error and is based on the $95 \%$ limits of agreement as described by Bland and Altman. ${ }^{1 \%, 18}$ it assesses whether an individual difference between 2 scores of a patient is a real change or whether it is a change that cannot be separated reliably from measurement error. So, progression scores smaller than the SDD cannot be distinguished reliably from measurement error. For clinical trials, it has been advised that the average scores of 2 observers be used, and therefore the SDD should based on interobserver differences. ${ }^{14}$ Consistent with this advice, we used the SDD based on the average scores of 2 observers. Differences in sensitivity for detecting clinically relevant changes by using the interobserver SDD as the threshold level between the Sharp/van der Heijde method and the Larsen/Scott method were analyzed with MCNemar chi-square tests for paired proportions, with a $\mathrm{P}$ value 
of 0.05 as significance level. Also the $95 \%$ confidence intervals $(95 \%$ Cl) of these differences, calculated according to Gardner and Altman ${ }^{19}$, were assessed.

Descriptive analyses, kappo statistics, intraclass correlation coefficients (ICC type 2.1) and MCNemar chi-square tests were performed with SPSS, version 10.0 for Windows (Chicago, 14 ). ROC curves were performed by MedCalc statistical soffware, version 6 for Windows (Brussel, Belgium).

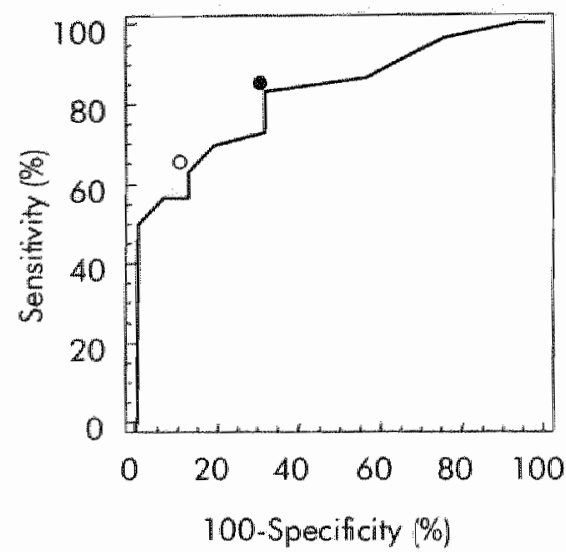

FIGURE 4.1

Example of the receiver operator characieristic curves used, comparing the accuracy of the Sharp/van der Heijde progression scores in patients with early rheumatoid arthritis and high disease activity with the opinion of the expert panel as external criterion

- = progression score with highest sensitivity/specificity combination (minimal clinically important difference; 3.0 units);

$0=$ smallest progression score that can be detected apart from measurement error (smallest delectable difference; 5.0 units)

\section{Results}

Opinion of the expert panel

Eighty percent of the sets of films showed progression of joint damage according to the opinion of the panel (Table 4.1). Whether they regarded this progression as clinically relevant depended an the clinical setting. Progression of joint damage was mainly considered clinically relevant in patients with early RA and high disease activity. Clinically relevant progression was seen in $65 \%$ of the 46 pair of sets, i.e., in $81 \%$ of the sets $(30$ of the 37$)$ considered by the panel to be showing progression. Only $22 \%$ of the sets, i.e., $27 \%$ (10 of the 37 ) of the "progressive" film sets, were labeled as clinically relevant in the setting with late RA and mild disease acrivity. Thus, progression of joint damage in a patient with 
early RA and high disease activity who was receiving methotrexate treatment was a reason to change treatment in $81 \%$ of the cases, compared with only $27 \%$ of the films sets from patients with late RA and mild disease activity. In the other 2 settings, early RA with mild disease activity and late RA with high disease activity, the expert panel reported clinically relevant progression in $46 \%$ and $50 \%$ of the seis, respectively, or in about $60 \%$ of the "progressive" sets of films.

\section{TABLE 4.1}

Prevalence of film sets with progression of joint damage in the hands and/or feet by the opinion" of the expert panel and the intra-panel reliability"

\begin{tabular}{|c|c|c|c|c|c|}
\hline & $\begin{array}{c}\text { Prevalence } \\
\text { of } \\
\text { progression } \\
\text { session l }\end{array}$ & $\begin{array}{c}\text { Prevalence } \\
\text { of } \\
\text { progression } \\
\text { session } 2\end{array}$ & $\begin{array}{c}\text { Concordan } \\
\text { apinon }\end{array}$ & $\begin{array}{l}\text { Observed } \\
\text { Agreement }\end{array}$ & Kappa \\
\hline & $\%(\mathrm{~N} / \mathrm{N})$ & $\%(n / N)$ & $\%(n / N)$ & $\%$ & \\
\hline Progression of joint damage & $80(37 / 46)$ & $87(40 / 46)$ & $76(35 / 46)$ & 85 & 0.45 \\
\hline \multicolumn{6}{|l|}{ CID by dinical setting } \\
\hline Early RA, mild DA & $46(21 / 46)$ & $39(18 / 46)$ & $33(15 / 46)$ & 80 & 0.60 \\
\hline Early RA, high DA & $65(30 / 46)$ & $59(27 / 46)$ & $52(24 / 46)$ & 80 & 0.59 \\
\hline Late RA, mild DA & $22(10 / 46)$ & $24(11 / 46)$ & $20(9 / 46)$ & 93 & 0.82 \\
\hline Late RA, high DA & $50(23 / 46)$ & $37(17 / 46)$ & $37(17 / 46)$ & 87 & 0.74 \\
\hline
\end{tabular}

The majority opinon of the panel was used for oll analyses. Comcordant opinion was defined as progiression seen in the 1 st as well as in the 2 id session, CID - cinically important eifference (between 2 pairs of hand and foot filmos), RA - rheumatoid arthritis, DA - disease detwity

Judgment of dinically important progression was quire consistent, as demonstrated by the same judgment by the panel at the first and the second round (concordant opinion), the percentages of abserved agreement between the first and second round and the chance-adjusted measurements of agreement (kappa). The panel seemed to be more consistent in judging the relevance of progression in late RA ( $\kappa .0 .82$ and 0.74 , in those with mild and high disease activity, respectively) than in early $\mathrm{RA}(\kappa \quad 0.60$ and 0.59 , respectively) (Table 4.1).

A quantification of the smallest progression of joint damage seen by the panel, expressed in scores, can be obtained by looking of the concordant opinion of the panel. The concordant, that is the most conservative, opinion of the panel was compared with the scores by the 2 scoring methods. Ninety-five percent of the progression was detected by the panel if a threshold of 5.0 units was used for the Sharp/van der Heijde method. A progression of 1.0 unit by the Larsen/Scott was picked up in all cases by the panel. 


\section{Radiographic scores}

Table 4.2 shows the distribution characteristics of both scoring methods (note that the units differ between the 2 methods). The distribution of scores was highly skewed, as is common with radiographic data.

\section{TABLE 4.2}

Distribution characteristics and interobserver reliability of the scoring methods between 2 observers"

\section{Sharp/ van der Heilde}

$(\max 448)$

\begin{tabular}{|c|c|c|}
\hline \multicolumn{3}{|l|}{ Baseline scores } \\
\hline Meari ISD & $24.6 \pm 16.5$ & $14.5 \pm 10.4$ \\
\hline Median & 19.5 & 15.3 \\
\hline Inter Quartile Range & $11.9-35.4$ & $5.8-19.8$ \\
\hline Range & $2.0-62.5$ & $0-37.5$ \\
\hline \multicolumn{3}{|c|}{ One-year progression in total scores } \\
\hline Mean $\pm \mathrm{SD}$ & $7.6 \pm 10.0$ & $4.0 \pm 8.0$ \\
\hline Median & 4.0 & 0.8 \\
\hline Inter Quartile Range & $2.4-8.6$ & 0.3 .6 \\
\hline Range & $0-51.0$ & $-3.5-43.5$ \\
\hline \multicolumn{3}{|l|}{ Interobserver reliability } \\
\hline SDD of progression scores & 5.0 & 5.8 \\
\hline ICC of progression scores & 0.94 & 0.88 \\
\hline
\end{tabular}

Larsen/Scott

$(\mathrm{max}, 200)$ 
resulted in correct detection of films with clinically relevant progression in $78 \%$ to $100 \%$ of the cases (mean $87 \%$ ). The Larsen/Scott method discriminated best between clinically relevant progression and no progression if threshold levels of $1.0-4.5$ (mean 2.3) were used, leading to sensitivity ranging from $60 \%$ to $83 \%$ (mean $75 \%$ ). The accompanying specificity ranged from $69 \%$ to $88 \%$ (mean $83 \%$ ) for the MCID of the Sharp/van der Heijde method, and from $83 \%$ to $100 \%$ (mean 90\%) for the MCID of the Larsen/Scott method. Overall, the Sharp/van der Heilde method was more sensitive and the Larsen/Scott more specific.

To test whether the differences in sensitivity and specificity between the 2 scoring methods were significant, the differences, with $95 \% \mathrm{Cl}$ of this difference, were calculated. The $95 \% \mathrm{Cl}$ of the difference in sensitivity between the scoring methods did not include zero only in the setting "early RA and high disease activity", indicating a significant difference in sensitivity to detect a clinical significant progression in favor of the Sharp/van der Heijde method in that setting ( $P=0.02)$. The differences in specificity did not reach significance in this study. The mean MCID of 4.6 Sharp/van der Heijde units and 2.3 Larsen/Scott units were both $\sim 1 \%$ of their maximum score.

As explained, the SDD can be used as an alternative threshold for clinically relevant progression (Table 4.4). For the Sharp/van der Heijde method, using the SDD (5.0 units) as the threshold resulted in a sensitivity ranging from $60 \%$ to $100 \%$ (mean $79 \%$ ) and specificity between $72 \%$ and $88 \%$ (mean $84 \%$ ). However, for the Larsen/Scott method, the SDD culoff value of 5.8 resulted in significantly lower sensitivity, ranging between $33 \%-48 \%$, except in the context of late RA and mild disease activity (80\%), and specificity between 94 and $100 \%$ (Table 4.4). In all settings, except late RA and mild disease activity, the $95 \%$ C.I. of the differences in sensitivity did not include zero, again indicating a significant difference in sensitivity for detecting a clinical significant progression in favor of the Sharp/van der Heijde method. The differences in specificity, which favored the Larsen/Scatt method, only reached significance if the methods were used in the context of late disease and mild disease activity.

Because the Larsen method and its modifications are strongly influenced by erosions, we also assessed the sensitivity and specificity for detecting clinically relevant progression by the Sharp/van der Heijde erosion score. Compared with the total score, the sensitivity of the erosion score was, on average, $24 \%$ lower, and ranged between 40 and $52 \%$, again except in the setting of late RA and mild disease activity $(90 \%)$. The specificity of the erosion score ranged between $92 \%$ and $100 \%$.

All analyses were repeated with the concordant opinion of the panel as standard. This led to results very similar to those presented here for the first reading only (data not shown). 


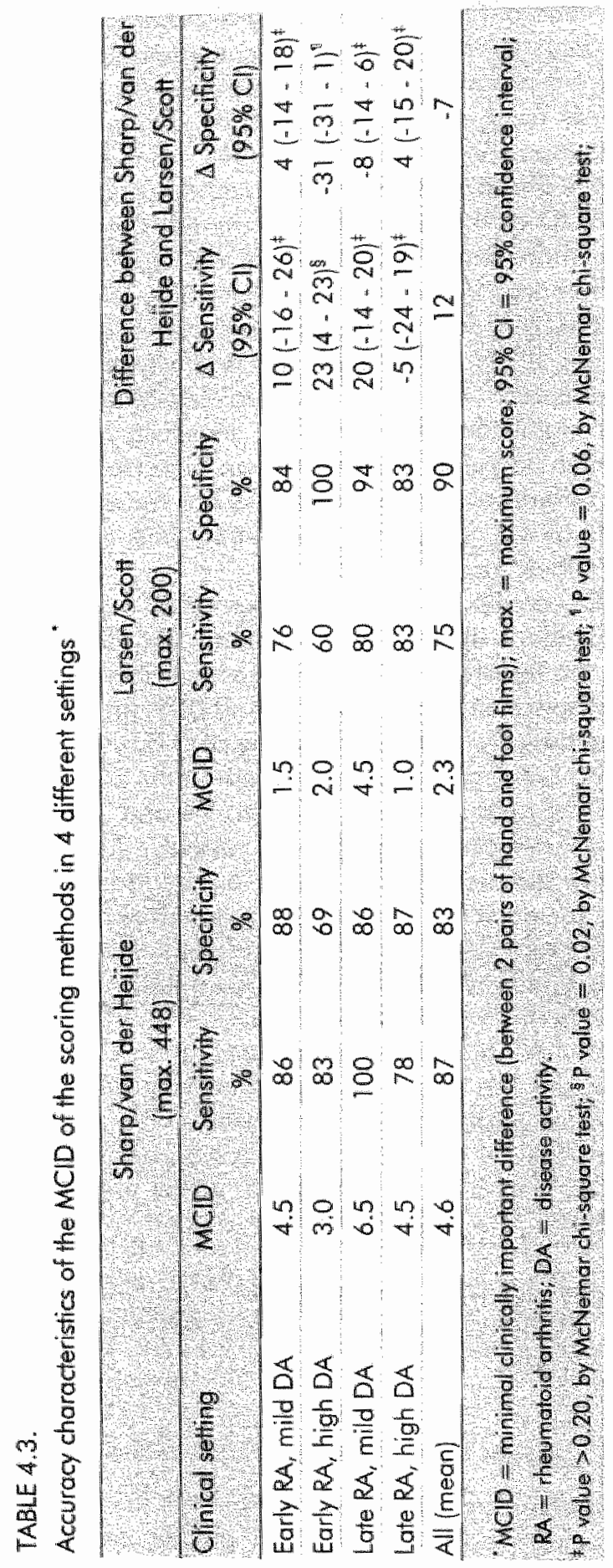




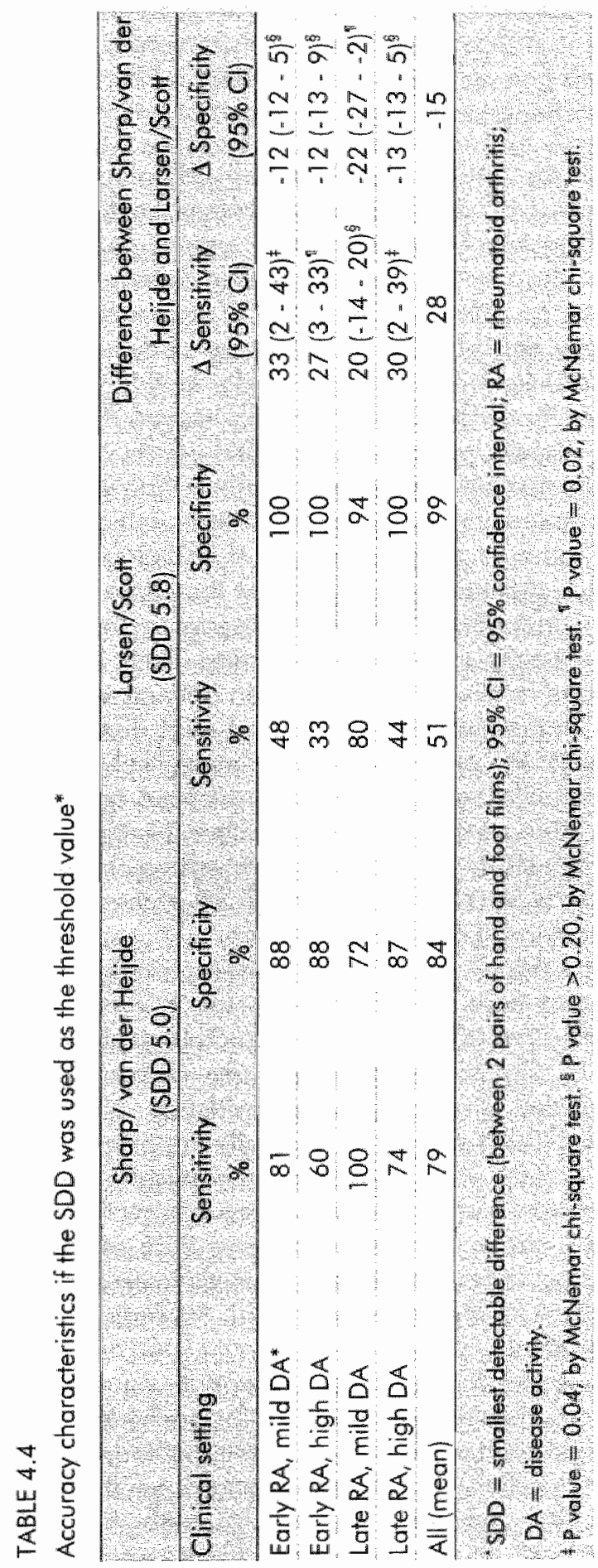




\section{Discussion}

This study assessed the MCID in RA-related radiologic joint damage as measured by the Sharp/van der Heijde and Larsen/Scatt scoring methods, by using the opinion of an infernational panel of experts as the external criterion. Such an opinion-based approach is a logical step forward in the delermination of the MCID and was accepted at OMERACT $V$ as an appropriate method to validate the statistically derived SDD. The present study showed that the MCID was, on average, 4.6 units for the Sharp/van der Heijle method and 2.3 units for the Larsen/Scott method. The MCID of the Sharp/van der Heijde method was almost equal to, or shightly lower than, its SDD, which was 5.0 units, but the MCID of the Larsen/Scott method was much smaller than its SDD of 5.8 units.

The MCD was assessed in 4 different clinical settings, because it was anticipated that the amount of progression of radiologic joint damage that makes theumatologists change treatment is not the same in patients with early RA and late RA and in patients with mild disease activity and high disease acivity, an the sense that theumatologists are more likely to define a change in radiologic cont damage as clinically important in patients with early RA and high disease aclivity than in patient with late RA and mild disease activity. This was bome out by the results: the panel was indeed more likely to judge progression as relevont in patients with early RA and high disease activity than in patients with late RA and mild disease activity, with the other 2 settings fitting in between. As a consequence, the MCID also differed per setting. However, the different MCID did nof exceed the SDD, except for the MCID of the Sharp/van der Heijde in the sefting wh the RA and mild disease activity.

The differences in the MCID according to disease setting raise the question whether the experts may have based their decision to change treatment mainly on clinical setting rather than on the extent of radiological change. However, to contradict this, the 10 film sets with progression exceeding the Larsen SDD, which can be regarded as unequivocal evidence of major radiological progression, were all $(100 \%)$ judged as clinically important in the setting early RA and mild disease activity. Thus the major radiological progression caused the experts to change treatment in all early RA cases irrespective of disease activity. in the setting late RA and mild disease activity, $80 \%$ of the sets with major radiological progression were also regarded as clinically impontant. Therefore, the decisions to change treatment were based on radiologic findings, albeit influenced by clinical setting.

Our results strongly suggest that it is appropriate to use the SDD of the Sharp/van der Heilde method as threshold for individual clinically relevant change in trials. However, for the Larsen/Scolt method, the SDD cutoff was insensitive for detecting clinically relevant changes on the individual level in patients with early RA and late RA patients with high disease activity. Considering the prospect of more powerful disease modifying antirheumatic 
drugs (DMARDS), only less progression of radiological joint damage will become acceptable, so greater detection of progression of joint damage will be required, i.e., more sensitive, rather than specific, scoring methods.

Two reasons come to mind to explain the results obtained using the Larsen/Scolt method. First, in this study, the method was less reproducible than the Sharp/van der Heijde, resulting in a relatively large measurement error (and thus, SDD). Second, the Larsen/Scott method may pick up less of the "fotal picture $^{t i}$ of the progression, that is, the information available on the radiograph as assessed by the clinicians: in other words the clinicians pick up more information from the films than is capiured in the Larsen/Scott progression score. However, applying a derivative of the measurement error, such as the SDD, as the threshold for the individual response makes sense from a methodological point of view. In this view the SDD, besides representing the smallest progression score that can be reliably separated from the measurement error, consequently also immediately guarantees the MCID. The SDD is in fact a statistically conservative estimate of the minimal important progression score. To complicate matters further, applying a statistically conservative estimate of the $M C I D$ as a response criterion in radiographic damage will lead to a higher proportion of patients being classified as showing no progression. From the clinical point of view this is the opposite of conservative, because we expect progression to occur.

The idea that clinicians pick up more evolution in the films than is captured by the Larsen/Scott progression scare is supported by the fact that by leaving the joint space narrowing out of the Sharp/van der Heijde scores, this method became nearly as insensitive in detecting clinically relevant progression as the Larsen/Scott. The rheumatologists apparently based their treatment decisions also to a large extent on joint space narrowing, on top of erosive damage.

Although the discrepancy between the SDD and MCID was especially large for the Larsen/Scott method, the panel also judged damage smaller than SDD of the Sharp/van der Heijde as clinically relevant. A possible explanation is that the experts also based their judgment on joints other than those incorporated in this scoring methods or on other features of joint damage in addition to erosions and joint space narrowing, e.g., periarticular osteoporosis or cysts.

The choice of our panel was, of course, a subjective one. We have chosen to use the majority opinion of an international panel of expert as external criterion in order to maximize the differences between the panel members and to represent the average expert. Both experts whose opinions are sensitive for detecting changes and those whose opinions are very specific are very important when composing a well-balanced panel. The intrapanel agreement on clinically relevant changes was satisfactory, as appears from the abserved agreement ranging from $80 \%$ to $93 \%$, and the chance-adjusted kappas, ranging form 0.59 to 0.82 . The lowest percentage of abserved agreement was in patients with early disease, although this was still $80 \%$. This finding is understandable, because already wery small changes were considered important in early RA. It is much easier to agree on large changes, as was the case in the setting with late RA, than on small ones. Because the agreement was also high in this setting and the 
results were comparable with the setting late $R A$ with high disease activity, it is unlikely that this somewhat lower agreement had a maior implication on the results. The kappa value for intrapanel agreement on progression alone, irrespective of its clinical relevance, on the other hand, was low $(0.45)$ and was in contradiction with the accompanying high level of observed agreement $(85 \%)$. The paradox "high observed agreement and low kappa" is a well-known feature of kappa statistics and is caused by the fact that kappas are affected by the prevalence of the disecise. ${ }^{20,21}$ Kappas can be misleadingly low despite good agreement in populations with very high or low prevalence of the disease. So, this low kappa probably does not reflect more uncertainty, but is coused by the high frequency of film sets showing progression of joint damage in our sample $(80 \%-87 \%)$.

The film sets used in this study were selected for high and low baseline scores and for high and low progression scores: to reflect the spectrum of damage found in RA trials. We stratified for baseline damage because it is hypothesized that the accuracy of the scoring methods for detecting progression of joint damage could depend on the amount of baseline damage. Moreover, estimates of stratified data, like ours, tend to be more precise than those abtained from random samples of the same size. The films were obtained from early RA patients treated with DMARDs, so that the progression rates are representative of what can be expected in current clinical practice. However it did not include many film sets with large progression of joint damage. Including such films would most likely improve reproducibility and thus decrease the SDD. The sample, although rather small, was large enough to detect differences between the Sharp/ van der Heijde and the Larsen/Scott methods.

In this study the MCID was defined as that progression in radiological joint damage that makes a rheumatologist change therapy. The decision to change therapy in practice, besides disease duration or activity, of course also depends on other factors like patient's history of toxicity, co-morbidity or availability of

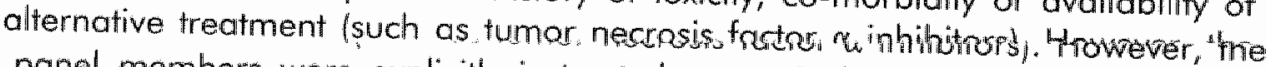
panel members were explicitly instructed not to include the above-mentioned factors in their decisions. We asked them to state their intention to change treatment, without taking into account other factors than the radialogical progression of joint damage and the specifications of the settings given.

Deriving MCID from a clinician's global assessment based on experience and knowledge is not the final step. A data-driven approach will definitely lead to the most scientific evidence for clinical relevance of a certain progression score of radiological joint damage, but will also be complex to obtain. Furthermore, it remains a difficult question whether the data-driven approach will provide unambiguous answers, particularly because outcome measures such as disability or sick leave also largely depend on factors external to the joint damage caused by RA. The determination of a MCID based upon clinical experts" opinions has also recently been described for hip osteoarthritis. ${ }^{22}$ This study showed that the experts' assessments of clinical importance of the joint space narrowing corresponded well with the SDD. In another study, the SDD 
predicted the need for a total hip arthroplasty. ${ }^{23}$ So combining these studies indicates that the expert opinion is a reliable and useful approach.

Use of the SDD as the threshold for clinically important progression in order to present trial data on an individuial level has recently been demonstrated in 2 trials. ${ }^{24,25}$ One of these studies even set the threshold as high as 15.5 Sharp/van der Heijde units. Analyses on group levels are of course more powerful to pick up difference between treatments and should always be done as primary analyses. ${ }^{2}$ However, the mentioned studies proved that anolyses on patient level actually can be powerful enough to discriminate between treatment groups in a trial. Reporting the proportion of patients who experience important improvement or deterioration will give additional information. ${ }^{20}$ This can also be used to calculate the so-called number needed to treat.

In conclusion, the expert panel judged changes in joint damage around the level of the SDD of the Sharp/van der Heijde method as minimal clinically important, so this study suggests that the SDD can be used as the MCID, i.e., as the threshold level for response criteria. However, since the expert-based MCID expressed in Larsen/Scott units was much smaller than the Larsen/Scott SDD based on 2 observers, the sensitivity of the Larsen/Scott SDD was too low to detect relevant progression. Biased on the resuls of this study, the SDD of the Larsen/Scott can therefore not be used as the threshold for individual relevant change or no change. 


\section{References}

1. Boers $M$, Tugwell $P$, Felson DT, wan Riel PL, Kirwan JR, Edmands JP, Smalen IS, Khalfaev $\mathbb{N}$, Muirden KD. World Health Organization and Intemational League of Associations for Rheumatology core endpoints for symptom modifying antirheumatic drugs in rheumatoid arthrilis clinical trials. I Rheumatol $1994 ; 41$ (suppl):86-9.

2. Van der Heijde D, Simon L, Smolen J, Lipsky P, Strand V, Breedveld F, Weisman M. Weinblatt $M_{r}$ Rau $R$, Lipsky $P$. How to repart radiographic data in randomized clinical trials in theumatoid arthritis? Guidelines from a roundtable discussion. Arthritis Rteum 2002;47:275-8.

3. Lassere MN, van der Heilde $D$, Johnson KR. Foundations of the minimal dinically important difference for imaging. J Rheumatol 2001;28:890.1.

4. Strand $V$, Lassere $M$, wan der Heide D, Johnson K, Boers M. Recent rheumatoid arthritis clinical trials using radiographic endpoints--updated research agenda. J Rheumatol $2001 ; 28: 887-9$.

5. Beaton DE, Bombardier C, Katz JN, Wright JG, Wells G, Boers M, sirand V, Shea B. Looking for important change/differences in studies of responsiveness. OMERACT MCID Working Group. Outcome Measures in Rheurnatology. Minimal Clinically Important Difference. J Rheumatol 2001;28:400-5.

6. Dougados M, Gueguen A, Nguyen M, Berdah L, Lequesne M, Mazieres B, Vignon E. Radiological progression of hip osteoarthritis: Definition, risk factors and correlations with clinical status. Ann Rheum Dis 1996:55:356-62.

7. Lassere $M$, Boers $M$, van der Heijde D, Boonen A, Edmonds J, Saudan A, Verhoeven AC. Smallest detectable difference in radiological progression. I Rheumatol 1999 ; 26:731-9.

8. Rawaud P, Giraudeau B, Auleley GR, Edoward-Noel R, Dougados M, Chastang C. Assessing smallest detectable chonge over time in continuous structural autcome measures: application to radiological change in knee osteoarthritis. I Clin Epidemiol $1999 ; 52: 1225-30$.

9. Bruynesteyn $K$, van der Heilde D, Boers M, Lassere $M$, Boonen A, Edmonds I, Houben $H$, Paulus $H$, Peloso P, Saudan $A$, wan der Linden S. Minimal dinically irmpontont difference in radiological progression of joint damage over 1 year in rheumatoid arthritis: preliminary results of a validation study with clinical experts. I Rheumatol 2001:28:904-10.

10. Van der Heijde D, Lassere $M$, Edmonds J, Kirwan J. Strand $V$, Boers M. Minimal clinically important difference in plain films in RA: group discussions, conclusions, and recommendations. OMERACT Imaging Task Force. I Rheumatol 2001;28: 914.7.

11. Van der Heijde D. How to read radiographs according to the Sharp/van der Heijde method. J Rheumatol 2000;27:261-3.

12. Scott DL, Houssien DA, Laasonen L. Proposed modification to Larsen's scoring methods for hand and wrist radiographs. British Journal of Rheumatology 1995;
$34: 56$. 
13. Van der Heijde DM. Plain $X$-rays in theumatoid arthritis: overview of scoring methods, their reliability and applicabillity. Baillieres Clin Rheumatol $1996 ; 10: 435 \times 53$

14. Van der Heilide D, Boonen A, Boers $M$, Kostense $P_{n}$ van Der Linden S. Reading radiographs in chronological order, in pairs or as single films has important implications for the discriminative power of rheumatoid arthritis clinical trials. Rheumatology 1999;38:1213-20.

15 Arnett FC, Edworthy SM, Bloch DA, McShane DJ, Fries JF, Cooper NS, Healey LA, Kaplan SR, Liang MH, Luthra HS, ef al. The American Rheumatism Association 1987 revised criteria for the classification of rheumatoid arthritis. Arthritis Rheum $1988 ; 31: 3151-24$

16. Metz CE. Basic principles of ROC analysis. Semin Nucl Med 1978; 8: 283-98.

17. Bland $J M$, Altman DG. Statistical methods for assessing agreement between two methods of climical measurement. Lancet 1986;1:307-10.

18. Bland JM, Alman DG. Measurement error. British Medical Jounal 1996; 313:744.

19. Gardner $M$, Altman D. Statistics with confidence: confidence interwals and statical guidelines. London: British Medical Journal; 1989.

20. Feinstein AR, Cicchetti DV. High agreement but llow kappa: I. The problems of two paradoxes. J Clin Epidemial 1990; 43: 543-9.

21. Cicchetti DV, Feinstein AR. High agreement but low kappa: II. Resolving the paradoxes. J Clin Epidemiol 1990; 43:551-8.

22. Maillefert $J_{r}$ Nguyen $M$, Gueguen $A$, Berdah $L$, Lequesne $M$, Mazieres B, Vignon $E$, Dougados $M$. Relevant change in radiological progression in patients with hip osteoarthritis. Part II: determination using an expert opinion approach. Rheumatology 2002;41:148-52.

23. Maillefent $J_{\text {r }}$ Gueguen $A, N$ guyen $M$, Berdah $L$, Lequesne $M$, Mazieres B, Vignon $E$, Dougados $M$. Relevant change in radiolagical progression in patients with hip osteoarthritis. Part I: determination using predictive validity for tatal hip arthroplasty. Rheumatology $2002 ; 41: 142-7$.

24. Lipsky PE, van der Heijde DM, St Clair EW, Furst DE, Breedveld FC, Kalden JR, Smolen JS, Weisman M, Emery P, Feldmann M, Harriman GR, Maini RN: AntiTumor Necrasis Factor Trial in Rheumatoid Arthritis with Concomitant Therapy Study Group. Infliximab and methotrexate in the treatment of rheumatoid arthritis. AnfiTumor Necrosis Factor Trial in Rheumatoid Arthritis with Conconitant Therapy Study Group. N Engl J Med 2000; 343: 1594-602.

25. Van der Heilde DM, van Riel PL, Nuver-Zwart $\mathbb{H}$, van de Pulte LB. Altemative methods for analysis of radiographic damage in a randomized, double blind, parallel group clinical trial comparing hydraxychloroquine and sulfasalazine. I Rheumatol 2000; $27: 535-8$.

26. Guyatt GH, Juniper EF, Watter SD, Griffith LE, Goldstein RS. Interpreting treatment effects in randomised trials. BMJ 1998; $316: 690-3$. 


\section{Chapter 5}

Progression of rheumatoid arthritis on plain radiographs judged differently by expert radiologists and rheumatologists

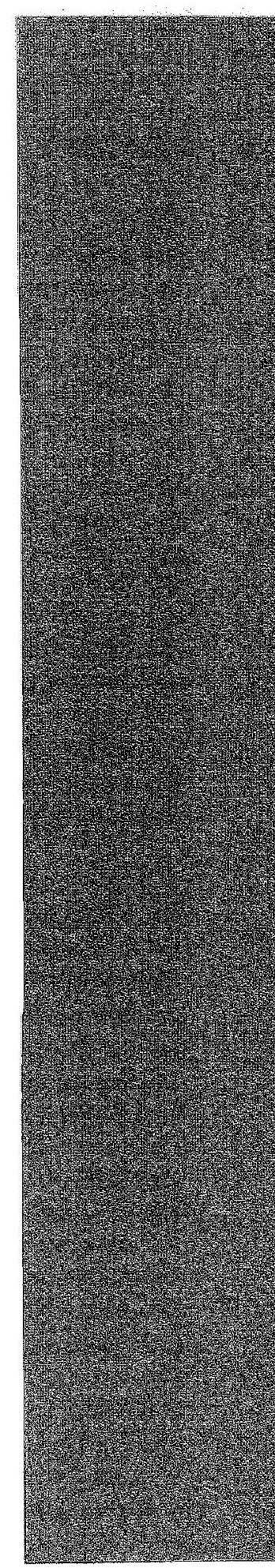




\title{
Abstract
}

\begin{abstract}
Aim
In a former study a panel of rheumatologists was used to assess which progression in radiological joint damage due to theumatoid artiritis (RA) an hand and foot radiographs taken at 1 years intervals was considered the minimally clinically impontant difference (MCID).
\end{abstract}

To compare the judgments of the parel of theumalologists with the judgments of two musculoskeletal radiologists.

\section{Methods}

Two experienced musculoskeletal radiologists evaluated independently the same hand and foot radiographs as assessed by the panel of rheumatologists. Progression was defined important if the radiologist would state it as substantial progression in their report. Two readers, different from the radiologists and rheumatologists, independently obtained the Sharp/van der Heijde scores. ROC analyses were performed to quantify the minimally impoitant progression defined by the radiologists expressed in Sharp/van der Heijde change-scores. The change-score with the highest accuracy represented the minimally important progression and was compared with the MCID defined by the panel of theumatologists for 4 different settings (early versus advanced RA and mild versus high disease activity (DA)).

\section{Results}

The minimally important progression defined by the radiolagists was estimated at 6.5 Sharp/wan der Heijde units. This was larger than the MCID defined by the panel of rheumatologists in 3 of the 4 clinical settings (3.0-4.5 units) and similar to the setting "advanced RA, mild DA". The panel of rheumatologists was inclined to change therapy in cases not reported as substantially progressive by the radiologists. The Sharp/wan der Haide progression scores of the radiographs on which the radiologists and rheumatologists disagreed related better with the rheumatologists opinion.

\section{Conclusion}

Changes that were not regarded as substantial by the radiologists were judged clinically important by the theumatologists in 3 of the 4 dinical settings. In this study, the radiologists appeared to be reserved in judging changes as important. 


\section{Introduction}

When analyzing clinical trials, the number of patients actually responding to the drug under investigation can provide important information, which adds to the information obtained from traditional statistical methods based on mean or median group changes. To assess whether a patient is a responder, a cutoff value needs to be chosen. In the ideal situation, the minimal clinically important difference (MCID) for the outcome measure in question is known, so that the outcome measure can be dichotomized. Several methods have been used to quantify which difference or change within an individual patient is clinically important. In a former study', we used the opinion of a panel of rheumatologists to assess the MCID for rheumatoid arthritis (RA) related radiological joint damage. Clinically relevant progression was defined in this study as the amount of progression of joint damage that would make the rheumatologists change the second line therapy prescribed. Because it was assumed that factors like disease duration and disease activity would influence this decision, the MCID was assessed for 4 different hypothetical settings: early RA with high disease activity, early RA with mild disease activity, advanced RA with high disease activity and advanced RA with mild disease activity. It was shown that the rheumatologists were more inclined to change therapy in patients with early disease and high disease activity than in patients with advanced RA and mild disease activity, with the other 2 settings fitting in between.

Several studies have shown that expert panels can be useful methods to estimate health changes in patients..$^{2.5}$ One part of the evaluation of the usefulness of the panels is to assess the consistency of the panels' judgments. Another aspect that should be investigated is the validity of the panel. Without the availability of a "gold standard", comparing the results with other outcome measures that assess adjacent health attributes can assess the so-called criterion or concurrent validity of a method. For panel judgments, an alternative for this is using the judgments of a panel composed of panelists of (an) other discipline(s). For radiological joint damage, a valid choice seems to be musculoskeletal radiologists. In daily practice, some rheumatologists rely on the judgment of a musculoskeletal radiologist. Although radiologists do not make clinical judgments, they do interpret the changes observed in their reports.

Aim of this study was to compare the results of the panel of rheumatologists used in a former study with the judgments of a couple of radiologists. In order to investigate how changes in radiological joint damage assessed as important by radiologists relate to the formerly defined MCIDs, we also estimated a minimally important difference defined by the couple of radiologists and compared it with the 4 previously assessed MCIDs defined by the panel of rheumatologists. Finally, this study gave us also the opportunity to investigate the influence of clinical information on the decision of the panel of rheumatologists whether progression noticed was considered clinically relevant or not. 


\section{Patients and Methods}

\section{Summary of methods of the former study"}

In this "MCID study" the expert panel consisted of 5 experienced rheumatologists of several nations. They independently evaluated 46 pairs of hand and foot radiographs, taken at 1-yeor intervals. They were first asked whether they noticed any progression of joint damage due to RA and if they noticed progression, they had to state whether they considered that difference clinically relevant in 4 hypothetical clinical settings: 1) Advanced RA and mild disease activity, 2) Early RA and mild disease activity, 3) Advanced RA and high disease activity and 4) Early RA and high disease activity. Clinically relevant progression was defined as progression of joint damage that would make the rheumatologist change the second line therapy methotrexate which has been started one year before. Radiographs were presented to the rheumatologist in chronological order. The majority opinion of the panel (3,4 or 5 out of 5) was the criterion applied in all analyses. The radiagraphs used in this study had been selected for high and low baseline joint damage and for high and low progression of joint damage. Radiographs were selected by an independent rheumatologist involved in the arganization of the COBRA trial. ${ }^{7}$ A selection was made to represent a wide spectrum of baseline joint damage and progression. All 46 patients fulfilled the 1987 American college of Rheumatologiy (ACR) classification criteria for RA. The inter observer reliability of the panel was assessed in the former study by average-measure intraclass correlation coefficients (ICC) and ranged between 0.60 (setting 4) and 0.74 (setting 3 ).

\section{Methods present study}

Two experienced musculoskeletal radiologists (FG and RW) evaluated the same radiographs as assessed by the rheumatologists. The radiologists were consultants with 5-10 years of experience in the field of musculoskeletal radiology. They judged the radiographs independently of each other. The radiologists were first asked whether they noticed any progression of joint damage due to RA and if they noticed progression, they further had to state during the same viewing session - whether they classified it as important progression, in the sense that they would record it as substantial progression in their report. As in clinical practice, the radiologists knew the chronological order of the hand and foot radiographs. No clinical information on disease duration and disease activity was given. The unanimous judgments of the 2 radiologists on the existence of (substantial) progression were used for the primary analyses. If one or both radiologists did not notice (substantial) progression of the joint damage, the radiographs were defined as non-(substantial) progressive. To estimate the intra observer reliability each radiologist viewed all radiographs twice, with an interval of at least 4 weeks. Sensitivity analyses were performed with the judgments of the radiologists defined as (substantial) progressive if one or both radiologists noticed (substantial) progression. 
To be able to quantify the radialogical progression (see also statistical analyses, below), the radiographs were also scored according the Sharp/van der Heijde ${ }^{6}$ method independently by 2 experienced readers, other than the radiologists or rheumatologists.' Both readers were researchers trained to score according the Sharp/van der Heijde method by dr. D. van der Heijde and have experience in scoring the radiograpgs of several trials. Radiographs were scored in chronological order and patients' identity was blinded. The Sharp/van der Heijde method assesses erosions and joint space narrowing separately and has a range from 0 to 448 . Thitty-two joints in the hands and 12 in the feet are scared for erosions, with a maximum score of 5 per joint in the hands and 10 per joint in the feet. Joint space narrowing is graded from 0 to 4 in 30 joints in the hands and in 12 joints in the feet. The principal score used in the analyses is the total score, which is the sum of the erosion score and the joint space narrowing score. Mean scores of the readers were used for the analyses.

\section{Statistical analyses}

The judgment on the presence of progression by the radiologists was compared with the judgment on the presence of progression by the rheumatologists with a two-by two table. Two-by-two tables were also made to describe the differences between the opinions on the importance of the progression by the radiologists with the opinion on the clinical importance of the progression by the rheumatologists. In order in to quantify the change in damage of the radiograph sets on which the rheumatologists and radiologists agreed and disagreed, the medians and interquartile ranges (IQR) of the Sharp/van der Heijde changescores were calculated for each cell of the two-by-two tables.

ROC analyses were performed to quantify the minimally important progression defined by the radiologists as Sharp/van der Heijde change-scares. The accuracy to discriminate between important progression and no (important) progression was assessed for every possible cutoff level of radiological joint damage expressed in Sharp/van der Heijde units. The ROC curve thus platted the true positive rate (sensitivity) in function of the false positive rate (100specificity) at all possible cutoff levels of radiological joint damage. The changescore with the highest accuracy for detecting important progression as defined by the radiologists represented the minimally important progression. Note that the change-score that discriminated best between important progression and no progression represented the minimally important difference and not the lowest progression score judged as important by the radiologists. This is because the latter would be $100 \%$ sensitive but not specific at all. Note further that this minimally important progression defined represents the minimally important progression of joint damage for an individual patient and not for a group of patients. The ROC analyses were performed by MedCalc statistical software. We compared the minimally imporiant difference defined by the radiologists with the 4 MCIDs defined by the panel of rheumatologists.

Single-measure and average-measure, random effects intraclass correlation coefficients (ICC), with $95 \%$ confidence intervals were calculated with SPSS 10.0 for Windows to evaluate the intra observer and inter observer reliability of the 
radiologists. The inter observer reliability between judgments of the panel of rheumatologists and those of both radialogists was also assessed by ICCs. For dichotomous outcomes, single-measures ICCs are equal to kappa statistics. Average-measure ICCs have the advantage that they can take into account whether the judgments of more than one observer or more than one viewing session is used in the analysis. Under the assumption that other radiologists would have had similar experience and training one can additionally simulate what the inter observer reliability (and thus generalizability) would have been if we used more than 2 radiologists (by dividing the variance components of the factor "radiologists" and its interactions by the simulated number of expert members). We simulated an expert panel that used the (majority) opinion of 1,3 and 5 radiologists.

\section{Results}

The single-measure intra abserver $\mathrm{ICC}$ of the judgments of both radiologists was $0.82(0.70-0.90,95 \% \mathrm{Cl})$ for the detection of progression of joint damage and $0.87(0.76-0.92)$ for the judgment of substantial progression. The averagemeasure inter observer ICC between the radiologists was $0.70(0.42-0.84)$ for the detection of joint damage and $0.82(0.67-0.90)$ for the judgment on substantial progression. These average-measures inter observer ICCs based on random effect variance components estimates the generalizability of the results to other pair of radiologists with similar experience and training. Using only one radiologist instead of 2 would have resulted in much lower inter observer ICC: simulations showed ICCs of 0.54 for the detection of joint damage and 0.69 for the judgment on substantial progression. The simulated ICC for the panels of 3 or 5 radiologists was 0.78 and 0.85 for the detection of joint damage and 0.87 and 0.92 for the judgment of substantial progression.

The radiologists labeled 20 of the $46(43 \%)$ sets as progressive. On 16 of these 20 substantial progression was seen, which is on $35 \%$ of all radiographs pairs $(16 / 46)$. Table 5.1 shows a two-way table in which the judgment on the presence of progression by the radiologists is compared with the judgment by the rheumatologists. All 20 sets judged as progressive by the radiologists were also labeled progressive by the rheumatologists. Seventeen sets judged as non progressive by the radiolagists were judged as progressive by the rheumatologists, which is $65 \%(17 / 26)$ af all sets judged as non progressive by the radiologists. To estimate the amount of change in damage of the 17 sets on which the rheumatologists and radiologists disagreed, the median Sharp/van der Heijde change-scores were determined (Table 5.2). A median change-score of 3.5 units was found for the 17 sets judged as non progressive by the radiologists but as progressive by the rheumatologists. A median change-score of 2.0 units was found for the radiograph pairs judged as non progressive by
both the radiologists and rheumatologists. 
TABLE 5.1

Judgment of the radiolagist on the presence of progression of joint damage compared with the judgment of the rheumatolagists

\begin{tabular}{|c|c|c|c|c|}
\hline \multicolumn{2}{|l|}{ 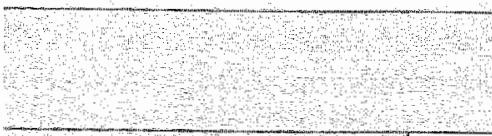 } & yes & Radiologists: progresion & toiral \\
\hline Panel of Rheumatologisis: & yes & 20 & 17 & 37 \\
\hline progression & no & 0 & 9 & 9 \\
\hline & total & 20 & 26 & 46 \\
\hline
\end{tabular}

TABLE 5.2

Median (interquartile range) Sharp/van der Heijde change-scores of the radiograph sets of each cell in Table 5.1

\begin{tabular}{|c|c|c|c|c|}
\hline & & yes & $\begin{array}{l}\text { ologists progres } \\
\text { no }\end{array}$ & on \\
\hline $\begin{array}{l}\text { Panel of Rheumatologists: } \\
\text { progiession }\end{array}$ & $\begin{array}{l}\text { yes } \\
\text { no } \\
\text { total }\end{array}$ & $\begin{array}{c}7.5(4.1-16.3) \\
- \\
7.5(4.1-16.3)\end{array}$ & $\begin{array}{l}3.5(2.3-6.0) \\
2.0(0.5-2.5) \\
2.5(1.4-4.4)\end{array}$ & $\begin{array}{l}5.5(3.0-13.0) \\
2.0(0.5-2.5) \\
4.0(2.4 \cdot 8.6)\end{array}$ \\
\hline
\end{tabular}

Tables 5.3a-d show two-way tables comparing the opinion of the radiologists on the importance of the progression seen with the opinion of the panel of rheumatologists for the 4 clinical settings. When considering a patient with advanced RA with mild disease activity (3a), the panel wanted to change treatment strategy in only 9 of the 16 cases $(56 \%)$ labeled as importantly progressive by the radiologists. The panel wanted to change treatment strategy in 1 of the 30 patients labeled as having no (important) progression by the radiologists. In the other 3 settings, the rheumatologists were inclined to change treatment in many patients that were not classified as important progressive by the radialogists. In patients with early RA with high disease activity (table 5.3d), the rheumatologists judged the amount of joint damage such that they even wanted to change therapy in 14 of the $30(47 \%)$ of the cases defined as not importantly progressive by the radiologist.

To quantify the agreement in judgments between the panel of rheumatologists and both radiologists inter observer ICCs were assessed. The single-measure inter observer $\mathrm{ICC}$ between the panel of rheumatologist and both radiologists was $0.42(0.15-0.63)$ for the detection of progression and ranged between 0.53 $(0.19-0.71)$ and $0.64(0.43-0.78)$ when comparing substantial progression defined by the radiologists with the clinical relevant progression defined by the rheumatologists.

To quantity the change in damage of the radiographs pairs on which the rheumatologists and radiologists agreed or disagreed, the median Sharp/van der Heijde change-scores were also determined for each cell of table 5.3a-d, 
shown in Tables 5.4a-d. These show that the median change-score ranged between 3.3 and 8.5 for the radiograph sets labeled as non-importantly progressive by the radiologist and as clinically importantly progressive by the rheumatologists. The median change-score of the radiographs set that made the rheumatologists change therapy, but which were not stated as importantly progressive by the radiologists, were higher than the median change-scores of the radiograph sets judged as important by the radiologists but did not result in change of treatment by the rheumatologists (difference of 0.5 to 3.3 in medians for the different settings). The median change-score for the radiograph sets judged as non-importantly progressive by both the radiologists and rheumatologists was 2.5 in all settings.

\section{TABLE 5.3}

Judgment of the radiologist on the importance of the progression* compared with the judgment of the rheumatologists on the clinical importance in 4 clinical settings

\begin{tabular}{|c|c|}
\hline $\begin{array}{l}\text { Ponel of rhewmatologists } \\
\text { Seting }\end{array}$ & $\begin{array}{l}\text { Radiologists } \\
\text { Sets with important progression }\end{array}$ \\
\hline & total \\
\hline
\end{tabular}

a: Advanced RA patients with mild disease activity

Sets with clinical important progression:

$\begin{array}{lccc}\text { yes } & 9 & 1 & 10 \\ \text { no } & 7 & 29 & 36 \\ \text { total } & 16 & 30 & 46\end{array}$

b: Early RA patients with mild disease activity

Sets with clinical important

$\begin{array}{lccc}\text { yes } & 14 & 7 & 21 \\ \text { mo } & 2 & 23 & 25 \\ \text { total } & 16 & 30 & 46\end{array}$

c. Advanced RA patients with progression: high disease activity

Sets with dinical important progression:

$\begin{array}{lccc}\text { yes } & 15 & 8 & 23 \\ \text { no } & 1 & 22 & 23 \\ \text { total } & 6 & 30 & 46\end{array}$

d: Early RA potients with high disease activity

Sets with clintical importan: progression:

$\begin{array}{lccc}\text { yes } & 16 & 14 & 30 \\ \text { no } & 0 & 16 & 16 \\ \text { lotal } & 16 & 30 & 46\end{array}$

important progression anount of pragression of joint damage staled as substantiol in the radiologists' teport clinically umportant progression, amount of progression of laint damage that would arake the theumatologist change the second - we therapy 
The ROC analyses showed that a cutoff level of 6.5 Sharp/van der Heijde units discriminated best between important progression and no important progression as assessed by the radiologists. Thus, the minimal individual change in radiological joint damage deemed important by the radiologists was estimated at 6.5 Sharp/van der Heijde units. In the former study the following MCIDs had been found: 3.0 units for patients with early RA with high disease activity, 4.5 units for patients with early RA with mild disease activity and patients with advanced RA with high disease activity and 6.5 units for patients with advanced RA with mild disease activity. The minimally important progression defined by the radiologists was larger than the MCID defined by the panel of rheumatologists in 3 of the 4 clinical settings and similar to the $4^{\text {th }}$ setting "advanced RA, mild disease activity".

\section{Sensitivity Analyses}

Sensitivity analyses were performed with (substantial) progression defined as positive if one or both radiologist noted (substantial) progression. As expected, more radiographs were now judged as (substantial) progressive: in $67 \%(31 / 46)$ one or both radiologists noted progression, and $50 \%(23 / 46)$ were labeled as substantial progressive. However, the majority of the sets judged as non progressive by the radiologist were still judged as progressive by the panel of rheumatologists $(53 \%, 8 / 15)$.

Regarding the importance of the progression noted, the sensitivity anallyses showed similar results when comparing the judgments of the radiologists with the judgments of the panel for the extreme settings (advanced RA with mild disease activity, early RA with high disease activity). In a considerable part $(13 / 23,56 \%)$ of the sets labeled as "substantial" progressive by the radiologists the rheumatologists were not inclined to change treatment if the patients had advanced RA and mild disease activity. But in patients with early RA with high disease activity, the rheumatologists were again inclined to change treatment in a substantial number of patients that were not classified as important progressive by the radiologists $(8 / 23,35 \%)$, despite the higher percentage of cases labeled as importantly progressive by the radiologists.

Comparing the radiologists' judgments with the judgments of the panel for the other 2 settings (patients with early RA with mild disease activity or patients with advanced RA with high disease activityl, the number of cases that were not regarded "substantial" progressive by the radiologists but in which the rheumatologists wanted a treatment change decreased in comparison with the primary analysis to 4 sets in both settings. However not all extra sets judged as important progressive by the radiologists were also judged as progressive by the rheumatologist. Furthermore, the median change-scores of the sets in which the rheumatologists did not want to change treatment but which were judged as substantial progressive by the radiologists were lower than those of the sets in which the rheumatologists wanted to change therapy but were not judged as important progressive by the radiologists $(3.0$ and 2.5 versus $5.0 \mathrm{SvH}$ units in both settings, respectively). The minimal individual change in radiological jaint 
damage deemed importantly by the radiologisłs became 4.5 Sharp/van der Heifde units in the sensitivity analysis, which equals the MCID defined by the panel of rheumatologists for the intermediate settings, but is larger than the MCID for patients with early RA with high disease activity and smaller than the MCID for patients with advanced RA with mild disease activity.

TABLE 5.4

Median (interquartile range) Sharp/van der Heijde change-scores of the radiograph sets of each cell in Table 5.3

\begin{tabular}{|c|c|}
\hline & $\begin{array}{l}\text { Sharp/van der Heijde change scores } \\
\text { (Median }(\text { QR })\end{array}$ \\
\hline Panel of heuniatologists & Radiologists: \\
\hline Setting & Sets with important progression \\
\hline & yes 3 no $\quad$ Toral \\
\hline
\end{tabular}

a: Advanced RA patients with mild diseose activity

Sets with dinical:

important progression ${ }^{*}$ :

$$
\begin{array}{llll}
\text { yes } & 14(9.8-23.5) & 8.5(8.5)^{\dagger} & 14(8.3-21.8) \\
\text { no } & 6.0(4.0-17.0) & 2.5(1.3-4.0) & 3.0(1.6-5.4) \\
\text { total } & 13(6.3-19.3) & 2.5(1.4-4.3) & 4.0(2.4-8.6)
\end{array}
$$

b: Eorly RA potients with mild disease activity

Sets with dinical important progression:

$\begin{array}{llll}\text { yes } & 14(7.4-21.8) & 5.0(3.5-8.5) & 8.5(5.5-16.3) \\ \text { no } & 3.5(3.0-4.0)^{\dagger} & 2.5(1.0-3.5) & 2.5(1.0-3.5) \\ \text { total } & 13(6.3-19.3) & 2.5(1.4-4.3) & 4.0(2.4-8.6)\end{array}$

C: Advanced RA potients with high disease activity Sets with dinical important progression:

$\begin{array}{ll}\text { yes } & 14(7.0-20.0) \\ \text { no } & 4.0(4.0)^{1} \\ \text { total } & 13(6.3-19.3)\end{array}$

$4.5 \cdot(3.7-7.9)$

$7.5(4.5-14.5)$ $2.5(0.9-3.5) \quad 2.5(1.0-3.5)$ $2.5(1.4-4.3) \quad 4.0(2.4-8.6)$

d. Early RA patients with high disease activity

Sets with clinical important progression:

$$
\begin{array}{llll}
\text { yes } & 13(6.3-19.3) & 3.3(1.9-6.6) & 6.5(3.1-14.1) \\
\text { no } & - & 2.5(0.6-3.5) & 2.5(0.6-3.5) \\
\text { total } & 13(6.3 .19 .3) & 2.5(1.4 .4 .3) & 4.0(2.4-8.6)
\end{array}
$$

\footnotetext{
* Important progressian, amount of progiression of loint damage stated as subistantial in the rodiologists repott dinieally impontant progression anount of progression of joint damage that would nake the theumotologists change the second line therapy prescribed, inedicun and interquartile ranged based on 1 or 2 radiographs sets.
} 


\section{Discussion}

That the panel of rheumatologists was inclined to change therapy in cases not reported as substantially progressive by the radiologists raises the question whether the rheumatologists based their decision to change therapy on the clinical information rather than on the extent of the radiological change. Clinical information was given to the panel of rheumatologists to evaluate its influence on the MCID for RA-related radiological jaint damage. Implicitly, we thus evaluated the influence of clinical information on the therapy strategies based on radiological joint damage. Apart from influencing therapy strategies, clinical information may also influence actual recognition of the features on the $x$-rays. Previous studies investigating the influence of clinical information on the interpretation of roentgen examinations have shown mixed results: an increase in true-positive rate ${ }^{8-10}$, an increase in false positives ${ }^{11,12}$, or just no effect. In our study, we first asked the panelists whether they abserved progression of joint damage or not. If they noticed progression, they were asked in the same session to judge whether they considered that level of progression clinically relevant for 4 clinical settings. Thus clinical information was only given after they viewed the radiographs. This makes variability in accuracy to observe progression due to clinical information most unlikely. Moreover, the rheumatologists were not only inclined to change therapy in cases not reported as substantially progressive by the radiologists, they also judged more radiographs as progressive than the radiologists did. These radiographs had a higher median Sharp/van der Heijde change-score than the radiographs judged as non progressive by both the radiologists and the rheumatologists. Apparently the radiologists in this study were more reserved in labeling radiograph sets as progressive than were the rheumatologists. This was also reflected in the fact that in the primary anallyses the minimal difference on hand and foot radiographs taken with 1-year intervals that was stated as substantial progression of joint damage by the radiologists appeared to be larger than the MCID for 3 of the 4 settings defined by the panel of rheumatologists and similar to the setting "advanced RA, mild disease activity".

A better explanation for the higher percentage of cases defined as dinically important progression in the settings with high disease activity or recently diagnosed RA can be the following. In patients with advanced RA with mild disease activity the rheumatologists did not consider minor changes as clinically important, but they did consider them clinically important in patients with early RA or patients with high disease activity. These "minor changes" were not considered as substantial by the radiologists. Another explanation may be that in patients with early RA or patients with high disease activity the rheumatologists were inclined to change therapy in case of "ambiguous changes" instead of "minor changes". So the clinical information might have introduced bias, namely so-called expectation bias ${ }^{13}$, they expect progression of joint damage in patients with active disease and therefor are inclined to judge "ambiguous" changes as "minor, but clinically important" changes if the patients have high disease activity. Evaluating the Sharp/van der Heilde progression scores of the 
pairs on which the radiologists and theumatologists disagreed, gave more insight in this matter. After all, the Sharp/wan der Heijde readers were blinded for patient's disease activity and disease duration. These analyses showed that change-scores of the sets judged as "positive" by the panel of rheumatologists and "negative" by the radiologists were higher than in the sets judged "positive" by the radiologist and "negative" by the rheumatologists. Furthermore, the medion change-scores of the radiograph sets iudged as "positive" by the rheumatologists and "negative" by the radiologists were for all 4 cases higher than the sets judged as "negative" by both the radiologists and the theumatologists. The judgments by the rheumatologists were thus supported by the independently obtained Sharp/van der Heijde scores, which have been documented to be related to outcome like physical functioning. ${ }^{14}$

In the sensitivity analyses the percentage of sets judged as (substantial) progressive was logically higher. Comparison of the radiologists' judgment with the rheumatologist's judgments, however, showed similar results for the extreme settings as compared to the primary analysis. For the intermediate settings, the percentage of patients in which the rheumatologists were inclined to change treatment but were not labeled "substantial progressive" by the radialogists decreased compared to the primary analyses. However, for the intermediate settings as well, the Sharp/wan der Heilde progression scores continued to relate better with the rheumatologists" opinion than with the radiologists".

To ensure the reliability and generalizability of an outcome measurement it is customary to use standardized scoring methods and well trained experts. When determining (clinically) important differences, however, standardization is of course not possible and the "training" occurred in the form of years of medical education and daily practice. In our former study it was therefore decided to use a panel of 5 rheumatologists instead of just 2 abservers, as is common in the field of scoring radiological joint damage due to RA. In this study, however, we only used 2 radiologists, because the opinion about important change was expected to differ less between radiologists than the opinions on changing a therapy strategy due to radiological joint damage progression by rheumatalogists. In addition, we anticipated that the high number of hand and foot radiographs seen daily by musculoskeletal radiologists would ensure consistency of their opinion. The intra observer and inter observer ICC of the radiologists were indeed moderate to good, and were higher than those of the panel of irheumatologists. However, because we realize that the number of radiologists is a limitation of this study we also simulated how the inter observer reliability wauld have been with 1,3 and 5 radiologists, under the assumption that these radiologists would have had comparable experience and training. These simulations showed that the greatest gain in generalizability was found by increasing the number of radiologists from 1 to $2(0.54$ to 0.70 and 0.69 to 0.81 for scoring progression and substantial progression respectively). By adding more radiologists the generalizability would have increased further, to 0.85 and 0.92 when, using the majority opinion of 5 radiologists, in comparison with 0.70 and 0.82 obtained in our study, based on 2 radiologists. 
When constructed properly, panels can give reliable estimates of health outcomes. Because non-standardized judgments of experts tend to wary widely, panels by definition contain more than one expert. For panels, formal consensus methods are often used to assess the health outcome in question. ${ }^{15}$ These consensus methods derive quantitative estimates through qualitative approaches. Part of the approach is to give the panelist feedback on the decisions made by other panelists. In the case of radiological joint damage due to RA in the hand and feet this is difficult to do. Such feedback namely urges to specify the joints judged by the panelists, which however resembles officially scoring of radiographs instead of non standardized judgments. The structure of an informal consensus meeting was also not thought to be appropriate because the risk of being dominated by the more powerful member(s). Therefore, the radiographs were judged independently and in the analyses the majority opinions of both panels were used. That the judgments of single panelists tend to vary and consequently consensus or majority opinion methods are used to express the health outcome in questions, means that the results of such panels do not lend themselves for inferences for clinical practice. . $^{5,15,16}$

Both the expert panell of rheumatologists and the radiologists were consistent over time. However, to determine whether the judgment of a panel of rheumatologists, besides producing a consistent outcome, is also a valid method of assessing the MCID, the panel results should ideally be compared with a gold standard. As such a standard is not available for radiological joint damage due to RA, an alternative method should be sought. In this paper we illustrated the comparison with the judgments of a panel composed of panelists of another discipline, namely radiologists. The radiologists could not be asked to derive inferences on the clinical importance of the progression, so they were asked to state whether they noted progression that they would state as substantial in their reports in daily practice. That there was an (unavoidable) difference in the definitions of relevant progression in the assessments of the radiologists and rheumatologists, however, is not likely the cause of the results found in this study. Moreover, the fact that the radiologists judged fewer radiograph sets as progressive, regardless of the clinical relevance, reveiled that the difference in definitions did not cause the differences found between the judgments of the 2 professions. However, which profession gave the most valid judgment remains debatable and cannot be definitely determined by this study. This study, however, did reveal that the type of profession can strongly influence panels' judgments and - although not feasible for research like ours in which the MCID is defined as that progression of the outcome measure that would make the professional want to change treatment of that patient- from a generalizability point of view it seems important to include more than one profession in an expert panel.

The concurrent validity of expert panels to assess MCID can also be assessed by comparing their judgments with the judgments of the actual patienis. In many cases, the judgments of the patients can even be considered the gald standard (i.e., in the case of pain and quality of life), but it is clear that patients cannot decide whether progression of radiological damage is clinically important or 
not. Although we realize that radiological joint damage is an intermediate outcome measure it is still belleved to be important to assess in trials in addition to the patient reported outcomes. From a research point of view, it is consequently important to estimate the clinical relevance of a certain progression score of radiological joint damage. Deriving the MCID from a clinician's global assessment based on experience and knowledge, however, is not the final step. A data-driven approach will have to lead to more scientific evidence for clinical relevance of a certain progression score of radiological joint damage. The question remains, however, which data-driven approach will provide unambiguous answers, particularly because outcome measures like disability also largely depend on foctors external to joint damage caused by RA. To conclude, in this study, the radiologists were consistent over time and with each other, and were able to differentiate patients with more progression from those with less progression, but were reserved in judging changes important compared to the panel of rheumatologists. It seems that minor changes that were not stated as substantial by the radiologists were judged to be clinically important by rheumatologists in patients with early RA, whatever the disease activity, and in patients with advanced RA with a high disease activity. 


\section{References}

1. Bruynesteyn $K$, van Der Heide D, Boers $M$, Saudan A, Peloso P, Paulus H. Houben H, Griffiths B, Edmonds J. Bresnihan B, Boonen A, van der Linden S. Determination of the minimal dinically important difference in theumatoid arthritis joint damage of the Sharp/van der Heijde and Larsen/Scoll scoring methods by clinical expents and comparison with the smallest defectable difference. Arthritis Rheum 2002:46: 913-20.

2. Bernstein SJ, Hofer TP, Meiller AP, Rigter H. Setting stundards for effectiveness: a comparison of expert panels and decision analysis. Int J Qual Health Care 1997;9: 255-63.

3. Maillefert JF, Gueguen A, Nguyen M, Berdah L, Lequesne M, Mazieres B, Vignon E. Dougados $M$. Relewant change in radialogical progression in patients with hip. asteaarthritis. 1. Determination using predictive validity for total hip arthroplasty. Rheumatology (Oxford) 2002;41:142-7.

4. Maillefert JF, Nguyen $M$, Gueguen A, Berdah L, Lequesne M, Mazieres B, Vignon E, Dougados $M$. Relevant change in radiological progression in patients with hip osteoarthritis. II. Determination using an expert opinion opproach. Rheumatology (Oxford) $2002 ; 41: 148,52$.

5. Hatvedt R, Lossius HM, Kristiansen IS, Steen PA, Soreide E, Forde OH. Are expert panel judgments of medical benefits reliable? An evaluation of emergency medical service programs. Int J Technol Assess Health Care 2003, 19:158-67.

6. van der Heijde D. How to read radiographs according to the Sharp/van der Heilde method. J Rheumatol 2000;27:261-3.

7. Boers $M$, Verhoeven AC, Markusse HM, van de Laar $M$, Westhovens $R$, wan Denderen JC, vam Zeben D, Dijkmans BA, Peeters AJ, Jacobs P, van den Birink HRi, Schouten HJ, van der Heijde DM, Boonen $A$, van der Linden S. Randamised comparison of combined step-down prednisolone, methotrexate and sulphasalazine with sulphasclazine a one in early rheumatoid arthritis. Lancet 1997; 350:309-18.

8. Doubilet P. Herman PG. Interpretation of radiographs: effect of dinical history. A.JR Am J Roentgenol 1981;137:1055-8.

9. Berboum KS, Franken EA, Jr., Dorfman DD, Barioon T, Ell SR, Lu CH, Smith W, AbuYousef MM. Tentotive diagnoses facilitate the detection of diverse lesions in chest radiographs. Invest Radiol 1986;21:532-9.

10. Berbaum KS, Franken EA, Jr., Dorfman DD, Lueben KR. Influence of dinical history on perception of abnormalities in pediatric radiographs. Acad Radio 1994:1:217. 23.

11. Eldevik OP, Dugstad $G$, Orrison WW, Haughton VM. The effect of clinical bias on the interpretation of myelography and spinal computed tomagraphy. Radiology $1982 ; 145: 85.9$.

12. Babcook CJ, Norman GR, Coblentz CL. Effect of dinical history on the interpretation of chest radiographs in childhood bronchialitis. Invest Radial 1993;28:214-7. 
Chapter 5

13. Brealey $S$, Scally AJ. Bias in plain film reading performance studies. Br I Radial $2001,74: 307-16$.

14. wan der Heijde D. Radiographic progression in theumatoid arthritis: does if reflect outcome? Does if reflect treatment? Ann Rheum Dis 2001;60 S3:iii47-50.

15. Jones J, Funter D. Consensus methods for medical and health services research. BM $1995 ; 311: 376.80$.

16. Vella K, Goldfrad C, Rowan K, Bion J, Black N. Use of consensus development to establish nafional research priorities in critical care. BMJ 2000;320:976-80. 


\section{Chapter 6}

Detecting radiological changes in rheumatoid arthritis that are considered important by clinical experts: influence of reading with or without known sequence

Bruynesteyn $K$, van der Heijde $D$, Boers $M$, Soudan A, Peloso P, Paulus H. Houben $H$. Griffiths B, Edmonds J, Besnihan B. Boonen A. van der Linden Si

I Rheumatol 2002:29(1)!:2306-12 


\section{Abstract}

\section{Aim}

To evaluate whether knowledge of the chronological sequence influences the sensitivity and specificity of the Sharp/van der Heijde (SvH) and Larsen/Scott (LS) scoring method to detect dinically important progression of joint damage caused by rheumatoid arthritis RAy in the indiwidual patient and assess whether scoring in chronological order leads to better sensitivity at the cost of lower specificity.

\section{Methods}

For both scoring methods, progression scores obtoined with (chronological) and without knowledge of the sequence of the films (paired) were compared with the judgment of an international expent panel. This panel assessed whether progression of joint damage seen on films with 7 year intervals was clinically relewant (defined as progression of joint damage that would make clinicians change therapy). The applied thresholds for clinical relevance were (1) the progression scores with the highest accuracy by receiver operating characteristics analyses for the expert opinion, and (2) the smallest pragression score that can be detected apart from interobserver measurement error by the scoring method, i.e., the smallest detectable difference (SDD).

\section{Results}

Progression scores that detected clinically relevant progression most accurately (chronological: 3.0 SvH units and 2.0 LS units; poired: 2.5 SvH units and 1.5 LS units) were smaller than the SDDs (chronological 5.0 SwH units and 5.8 LS units; paired 13.8 SvH units and 9.7 LS units: With the SDDs as threshold, the sensitivity to detect dinically relevant progression increased significantly from $20 \%$ to $60 \%$ for the SVH method and from 23 ro $33 \%$ for the 45 method if the sequence of the films was known. The specificity remained good when scoring chronologically: $88 \%$ for the SWH and $100 \%$ for the LS.

\section{Conclusion}

Our results suggest that knowing the chronological sequence leads to an increase in detecting clinically relevant changes in the patient without serious overestimation of nonrelevant differences. Analyzing a clinical trial should be done preferably by reading films in chronological order. 


\section{Introduction}

In rheumatoid arthritis (RA), several scoring methods have been developed to quantify radiological damage in the joints of the hands and feet. In longifudinal studies, the order in which films are presented to the observer influences results. ${ }^{1-4}$ Roughly, one can distinguish 3 ways of ordering films obtained at 2 or more points in time. First, films can be presented to the reader completely at random, i.e., a single film at a time. Second, films can be grouped per patient and presented to the reader without data on the chronological order of the films, which we call paired scoring. Finally, films can be grouped per patient and presented in chronological arder.

Reading single films randomly has the major drawback that the reader cannot compare with contralateral joints or with identical joints taken at other moments. Hence, the reader will not be able to correct for variation in positioning of the hands and feet or for film quality, which may contribute to the introduction of measurement error. In 1986, Fries et al.' demonstrated that precision of paired scaring was greater than reading single films randomly. Two Italian studies confirmed this finding in 1997.,3 Chronological reading provides the reader with a maximum of information, thereby reducing measurement error. Theoretically, reading films chronologically results in an increased ability to defect changes than the paired reading order. Van der Heijde et al. ${ }^{4}$ showed in 1999 that reading in chronological order was most sensitive to change. However, the possibility that the chronological reading order overestimated progression of joint damage because the readers expected to see progression over time could not be excluded. In other words, it could not be excluded that the extra signal picked up by the chronological reading order was actually a false signal caused by expectation bias; especially because the Sharp/van der Heijde $(\mathrm{S} v \mathrm{H})$ progression scores used could not decrease by definition when applied chronologically. ${ }^{5}$

An appropriate method to distinguish between a more precise signal, by reducing the measurement error, and a false signal (bias) is to compare the progression scores of the chronological and paired reading order of the scoring methods with an external criterion for progression of joint damage. The amount of progression that would make clinicians change therapy, in other words the amount of progression that is considered clinically important, can be regarded as a relevant external criterion for this purpose. In routine clinical practice films are judged with known sequence of the films. Hence, the judgments of a panel of rheumatologists aware of the sequence seems intuitively most appropriate as external criterion. However, overestimation by the rheumatologists when judging films chronologically can of course not be excluded either, although decisions on therapy changes are presumably made with prudence, making overestimation of clinically relevant progression less likely. All things considered, it was decided to present the films to the panel with and without information on 
the sequence of the films. However, before analyzing the 2 readings, the validity and reliability of the chronalogical and paired panel must be examined.

Our aim was to evaluate the influence of the paired and chronological reading order on the ability of the SvH $\mathrm{H}^{5}$ and Larsen/Scott (LS) scoring methods (6) to detect clinically important changes on radiographs deemed important by an external panel of clinicians. This in order to assess whether scoring in chronological order leads to better sensitivity at the cost of lower specificity.

\section{Patients and Methods}

\section{Method}

The judgments of an international panel of rheumatologists on the clinical relevance of progression of joint damage seen on sets of films with 1 year intervals was used as external criterion. The majority opinion of $5(3,4$ or 5 out of 5) clinicians was compared with the paired and chronological progression scores of the SvH and LS method, each obtained from 2 different pairs of observers. The same expert members, films and SvH and LS readers have been used in another study. The subject of that study was the minimal clinically important difference (MCID) of the SYH and the LS scoring method defined by clinical experts. ${ }^{6}$

\section{Expert panel}

The expert panel consisted of 5 rheumatologists (BB, BG, HH, HP and PP) who independently evaluated 46 pairs of hand and foot films, taken at 1 year intervals, of patients with early RA with varying followup duration (see also the section patients and films). The experts were chosen from several different countries based on their expertise in the treatment of RA. None had been trained in either of the scoring methods, but each was experienced in reading films in daily practice. The panel experts were first asked whether they noticed any progression of joint damage due to RA between the 2 sets of hand and foot films in 1 pair of films. Second, if they noticed progression, they had to state whether they considered that difference in joint damage clinically relevant in a typical early RA patient: a 46-year ald woman with a 2 year history of RA, with high disease activity, treated with methotrexate for 1 year. Clinically relevant progression was defined as that progression of joint damage that would make a clinician change second line therapy. In the original study, the panel also considered 3 other clinical scenarios.? As the results with these scenarios did not add relevant information for this study, they have been omitted here. Each panel expert viewed the radiographs 4 times: twice in chronological order and twice in paired order, at an interval of at least 4 weeks, to estimate the intrapanel reliability (variability between the first and second reading of the panel). Three panel members started with the chronological reading order, the other two with
the paired reading order, to minimize possible bias caused by learning effects.
The order in which patient sets of films weree The order in which patient sets of films were presented was different in the 4 
viewing sessions. Unlless stated otherwise, the opinion of the first viewing session of both reading orders was used in the analyses.

\section{Radiographic scoring methods and observers}

Two experienced readers scored the radiographs with and without knowledge of the sequence of the film, according to the SvH (DvH and AB) and LS method (JE and AS). Two different readers were used for each scoring method, because the readers can not be experienced in both scorning methods at the same time. The $\mathrm{SvH}$ method assesses erosions (hands $0-5$; feet $0-10$ ) and joint space narrowing $(0-4)$ separalely and has a range from 0 to $448 .^{5}$ The LS method has a range from 0 to 200 for hands and feet and applies one grade $(0-5)$ to each joint. ${ }^{s}$ The wrists are evaluated as single joints and are weighted by a factor 5 . The chronological SvH method was applied with the rule that scores cannot decrease by definition. The scores of the chronological LS and the scores of the paired reading orders of both scoring methods could however decrease in time. The mean scores of each pair of observers were used for further analyses.

\section{Patients and films}

The film sets of a recent study on precision and sensitivity to change of the SvH method were used in this study. ${ }^{4}$ The 46 pairs of hand and foot fillms, made in posterioanterior view, were obtained from 22 patients. The films were selected in the previous study for high and low baseline scores and for high and low progression scores between the 2 sets of 1 pair of films. All patients fulfilled the 1987 ACR classification criterion for RA and had a disease duration of $<1$ year at start. Ten patients had had a follow-up period of 1 year and supplied 1 pair of films each, 12 patients had had a follow-up period of 3 years and supplied 3 pairs each.

\section{Statistical analyses}

Receiver operating characteristics (ROC) curve analyses assessed the sensitivity and specificity of all available progression scores of the different reading orders of the 2 scoring methods to discriminate between clinically relevant progression and no (relevant) progression using judgment of the experts as standard. ${ }^{9}$ A ROC curve plots the true positive rate (sensitivity) in function of the false positive rate (100-specificity) at all possible cutoff levels. An overall index of goodness of the test, i.e., scoring method, is the area under the curve (AUC). A nondiscriminating test has an area of 0.5 and a perfect discriminating test an area of 1.0 . The threshold level that discriminates best is the progression score with the highest accuracy, i.e., that progression score with the best combination of sensitivity and specificity. In the ROC curve this is the cutoff point nearest the upper left corner. A second analysis assessed the sensitivity and specificity of the 2 reading orders if the smallest progression score that can be detected apart from measurement error, the smallest detectable difference (SDD), was applied as threshold. The SDD is a statistical method that is based on the $95 \%$ limits of 
agreement as described by Bland and Altman. ${ }^{10.12}$ This method helps to decide whether a difference between 2 scores of an individual patient is a real change, or one that cannot be separated reliably from differences caused by random variability (measurement error). Progression scores smaller than the SDD cannot be distinguished reliably from measurement error. Consequently, a threshold value should at least exceed the SDD, the measurement error, in the study in question. In clinical trials it has been advised to report radiographic results as the mean score of 2 observers; in line with this, we applied the SDD based on this mean score of change. Besides being specific for the sets of films involved, the magnitude of this SDD also depends on the quality of the specific observers and on whether one wishes to generalize to other pairs of observers. The SDD applied in this study was restricted to the current pairs of observers. It has been shown that the interobserver SDD, restricted to the same observer pair represents clinically relevant changes for the chronological reading order of both scoring methods: in the previous study with the same observers changes even smaller than these SDD were already considered clinically important by our panel. $^{7}$

Descriptive analyses, kappa statistics and intraclass correlation coefficients (ICC type 2,1) were performed by SPSS 10.0 for Windows. ROC curves were performed by MedCalc statistical software (Mariakerke, Belgium). For both scoring methods, McNemar chi-square tests for paired proportions compared the accuracy (sensitivity/specificity) of paired and chronological reading methads at the 2 thresholds (optimum accuracy and SDD). The 95\% confidence intervals of the differences in accuracy were calculated according to Gardner and Altman. ${ }^{13}$ A 2 -sided $P$ value of $\leq 0.05$ was considered significant.

\section{Results}

\section{Expert panel opinion}

Table 6.1 shows the prevalence of film sets with (clinically important) progression of joint damage in the hands and/or feet according to the chronological and paired panels. The prevalence of the film sets judged by the expert panel as progressive dropped from $80 \%$ to $37 \%$ if the sequence of the films was not known by the experts. The number of sets with elinically relevant progression decreased or increased to the same extent. However, the reliability of the paired panel did not decrease the same way, demonstrated by the kappas, reflecting the variability between the first and second reading of the panel (Table 6.1). When the sequence of the films was not known by the panel members, the panel members refused en masse to give a judgment on the (relevance of the) progression unless the changes were huge. Consequently this makes it impossible to use the paired panel to evaluate the ability to detect dinically relevant change by the paired progressions scores, i.e., to evaluate the change scares as threshold for minimal clinically relevant change, which is the
purpose of the study. 

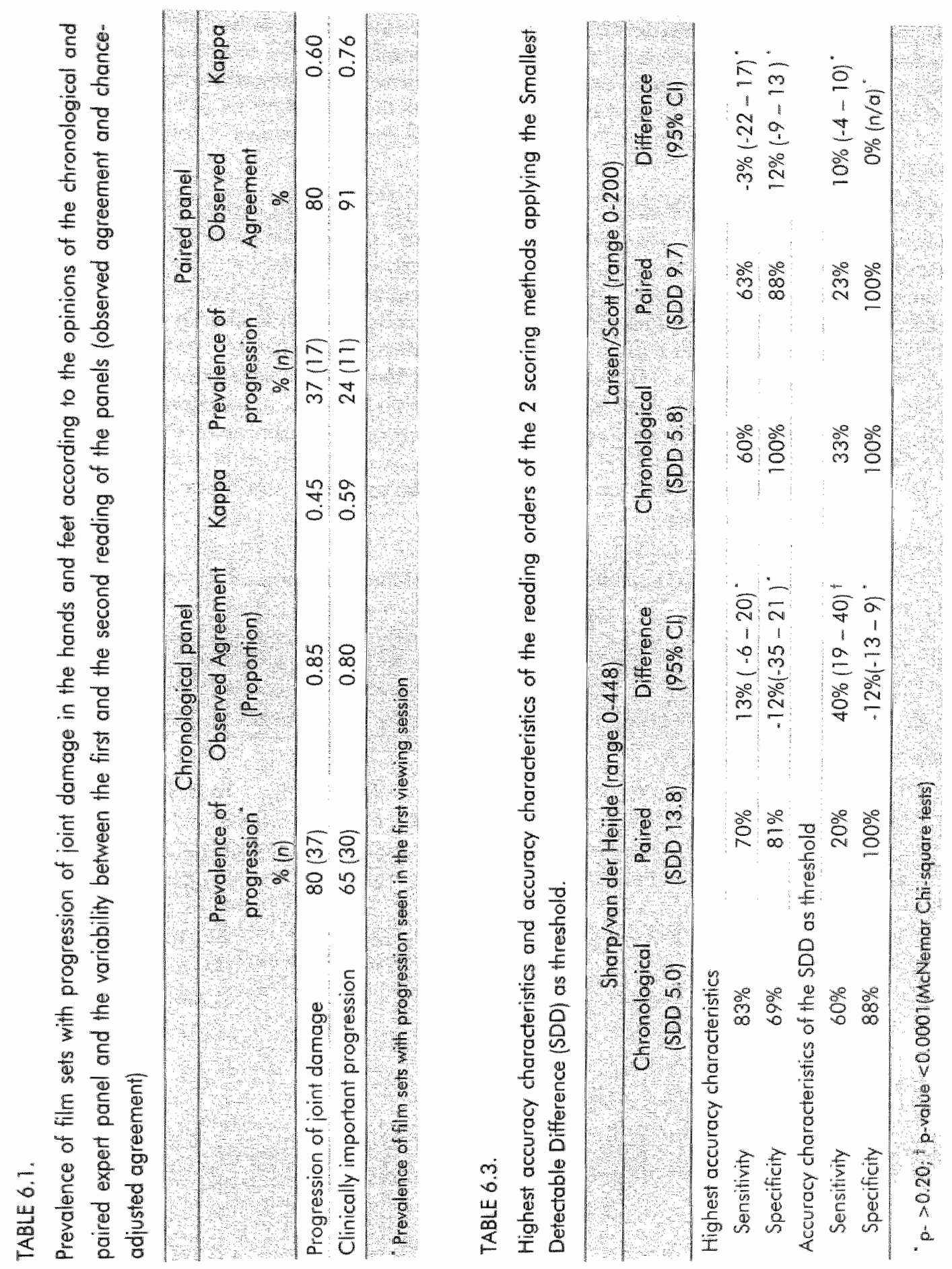
These results made us decide that clinicians really are experts only if films are presented to them in chronological order. Consequently, only the opinions of the experts judging chronologically ardered sets of films were evaluated further and discussed in this article.

\section{Radiographic scores}

Table 6.2 shows the distribution characteristics of the 2 reading orders for both scoring methods (note the different maximum obtainable scores). The chronological progression scores of the SvH method were evidently higher than the paired progression scores. The mean chronological SuH progression score was 7.6 (SD 10.0), the median 4.0 [inter quartile range (IQR): $2.4-8.6$ ]; that is $1.7 \%$ and $0.9 \%$ of the maximally abtainable score, respectively. The mean paired SvH progression score was 4.5 (SD 10.2), the median 2.5 (IQR: - 1.07.6); $1 \%$ and $0.6 \%$ of the maximally obtainable score, respectively. The difference between the paired and chronological progression scores of the SuH method was statistically significant ( $P=0.001$ Wilcoxon signed rank test). The influence of the reading orders on the LS progression scores was not that clear.

\section{TABLE 6.2}

Distribution characteristics and interobserver reliability of the different reading orders of the 2 scoring methods

\begin{tabular}{|c|c|c|c|c|}
\hline \multirow{2}{*}{ 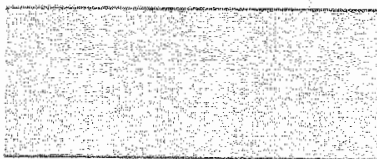 } & \multicolumn{4}{|c|}{$\begin{array}{l}\text { Sharp/ van der Heilde method Larsen/ Scolt nethod } \\
\text { (range } 0-448 \text { ) }\end{array}$} \\
\hline & Chronological & Paired & Chronological & Paired \\
\hline \multicolumn{5}{|l|}{ Baseline scores } \\
\hline Mean $(\mathrm{SD})$ & $24.6(16.5)$ & $25(116.0)$ & $14.5(10.4)$ & $16.8(11.2)$ \\
\hline Median & 19.5 & 21.5 & 15.3 & 15.8 \\
\hline IQR & $11.9-35.4$ & 14.4 .31 .8 & $5.8-19.8$ & $9.4-23.1$ \\
\hline Range & $2.0-62.5$ & $1.5-59.0$ & $0.0-37.5$ & $0.0-52.0$ \\
\hline \multicolumn{5}{|l|}{ Progression lotal scores } \\
\hline Mean (SD) & $7.6(10.0)$ & $4.5(10.2)$ & $4.0(8.0)$ & $3.7(10.3)$ \\
\hline Median & 4.0 & 2.5 & 0.8 & 1.0 \\
\hline IQR & $2.4 \cdot 8.6$ & -1.0 .7 .6 & 0.3 .6 & $-0.6-5.9$ \\
\hline Range & $0-51.0$ & -13.0 .52 .0 & $-3.5-43.5$ & $-12.0-55.0$ \\
\hline \multicolumn{5}{|l|}{ Interobserver reliability } \\
\hline SDD progression score & 5.0 & 13.8 & 5.8 & 9.7 \\
\hline KC progression score & 0.94 & 0.63 & 0.88 & 0.80 \\
\hline
\end{tabular}

IQR interquartile range, SDD. smallest deteciable difference

The mean chronological progression score of the LS method was 4.0 (SD 8.0), the median 0.8 (IQR: $0.0-3.6$ ), i.e., $2 \%$ and $0.04 \%$ of the maximally obtainable 
score. The corresponding figures for the paired LS progression scores were 3.7 (SD 10.3) and $1.0(-0.6-5.9)$, i.e., $1.8 \%$ and $0.05 \%$ of the maximally obtainable score. The agreement between the 2 readers of each scoring method diminished if the order in time was not known by the readers, shown by the increase in SDDs and decrease in intraclass correlation coefficients (Table 6.2).

Influence of the reading order on the sensitivity and specificity to detect clinically important differences in the individual

Figure 6.1 shows the ROC curves of the chronological and paired progression scores of the SvH and LS scoring method. The SVH AUC for the chronological reading order was 0.83 and for the paired order 0.82 . The LS AUC were 0.84 and 0.79 , respectively. So the AUC of the scoring methods were virtually identical and quite acceptable for both reading orders.

1a.Sharp/van der Heijde

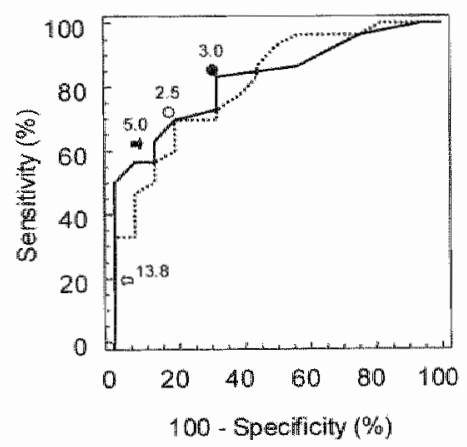

1b. Larsen/Scott

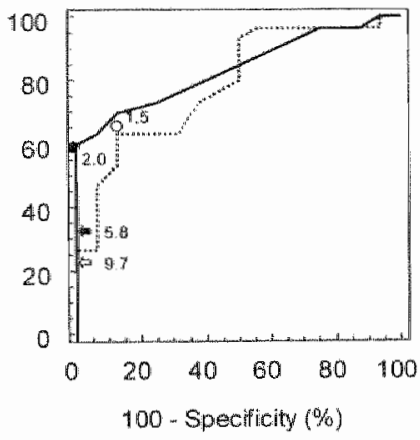

Figure 6.1. Receiver operator characteristic (ROC) curves of the chronological and paired progression scores of the Sharp /van der Heijde (la) and the Larsen/Scott (1b) scoring method, using the opinion of the expert panell as external criterion

Black line in curve : chronological reading order; datted line in curve: paired reading order;

Black dot: chronological progression score with highest accuracy; white dot: paired progression score with highest accuracy; black arrow: SDD chronological progression scores; white arrow:SDD paired progression scores; Sensitivity: percentage of patients with clinically important progression of damage correctly labeled by the scoring method (true positive rate); Specificity: percentage of patients without clinically important progression of damage correctly labeled by the scoring method (true negative rate); accuracy: combination of sensitivity and specificity; SDD: smallest detectable difference 
At the most accurate threshold for dinically important progression, little accuracy was last when moving from chronological to paired reading in both scoring methods (Table 6.3). The thresholds were slightly lower for the paired reading order (2.5 SvH units and 1.5 LS units) than for those of the chronological order ( 3.0 and 2.0 , respectively), like the majority of other progression scores.

At the SDD threshold for dinically important progression the results were different (Table 6.3). Because the SDD of the paired reading order was much higher than that of the chronological order, applying this threshold resulted in a decrease in sensitivity of both the SvH method $(40 \%$, from $60 \%$ to $20 \%)$ and the LS method $(10 \%$, from $33 \%$ to $23 \%)$ and an increase of specificity of the SuH $(12 \%$, from $88 \%$ to $100 \%)$. The difference in sensitivity between the chronological and paired SuH was highly statistically significant $(\mathrm{P}<0.0001)$, in contrast to the other differences.

\section{Sensitivity analysis}

One might argue that the paired reading order of the SwH was too insensitive because our SwH readers were not experienced enough in scoring films in paired order and as a result disagreed too much with each other, in contrast with Larsen readers. Therefore, we also analyzed the accuracy of the paired scoring method if the readers would have agreed more often and as a consequence the SDD would have been smaller. An imaginary SDD of 8.5 units was chosen, based on the ratio of $1.7(9.7: 5.8)$ between the paired (9.7) and chronological SDD (5.8) of the LS method. With this SDD as threshold the sensitivity of the paired $\mathrm{SvH}$ increased to $33 \%$.

All analyses were repeated with the concordant opinion of the panel as standard (i.e., progression seen in the first as well as in the second session by the majority of the panel), which led to results very similar to those presented here for the first reading only (data not shown).

\section{Discussion}

Underlying this study was the understanding that chronological reading order seemed to be more sensitive to change, but that this increased sensitivity might be due to expectation bias rather than diminishing of measurement error. In order to assess if the extra signal picked up by reading in chronological order was principally due to bias or indeed represented a more precise signal, we determined the influence of the paired and chronological reading order on the ability of the SwH and LS scoring methods to detect clinically relevant progression (defined by an expert panel) of radiological damage of hands and feet in the individual patient. To make a totally fair comparison between the reading orders and the opinion of the panel, we decided first to present the films to the expert members with and without information on the sequence of the films. A priori we considered the judgment of the panel that was not aware of the sequence not appropriate as external criterion. The results in Table 6.1 supponted this view 
and allowed us to abandon the paired panel as external criterion for the paired reading order. Clinicians are not experts in judging clinical relevance of progression if the chronological sequence is not known. If they don't know the chronological sequence they are only willing to change therapy if they are absolutely sure that the damage worsened, i.e., a large difference that uncovers the sequence. The somewhat higher percentage of observed agreement of the paired panel is also a reflection of this: it is much easier to agree on large changes than on small ones.

A possible overestimation of progression by the chronological reading order would result theoretically in higher sensitivity at the cost of lower specificity. At their most accurate threshold, the accuracy of paired and chronological reading orders was roughly similar for both scoring methods. However, these thresholds comprised progression scores that were smaller than the SDD and can thus not be distinguished reliably from measurement error in the individual case. Without knowledge of the sequence, scoring became less reproducible, increasing measurement error and thus the SDD. If the SDD was subsequently applied as threshold for clinically relevant progression, sensitivity of paired reading proved remarkably lower than that of chronological reading, especially for the SvH methad. As the specificity of chronological reading by this method was aliready good $(88 \%)$, the increase to $100 \%$ specificity by paired reading still represents only a modest gain. Hence, the advantage of higher sensitivity to pick up change caused by less measurement error surpassed the adverse consequence (loss in specificity) by the possible intraduction of bias. At $33 \%$, the sensitivity of the chronological LS method was already much less than that of the SvH method, and this decreased further to $23 \%$ in paired reading. Specificity remained perfect regardless of reading order. With the prospect of more powerful disease modidying antirheumatic drugs, future trials will document very low rates of progression or arrest of joint damage, thus requiring increasingly sensitive methods. This would be an argument for the chronological SvH scoring method.

The van der Heijde madification of the Sharp score included the rule that the scores could not decrease by definition if scoring in chronological order. This was based on the frequent experience that at one moment erosion would be clearly visible, at the next it would be gone, only to reappear at the next assessment. Theoretically the erosion could of course have healed at moment 2 and reappeared at moment 3 , but this was thought unlikely from pathophysiological point of view. Rather, variation in film quality or positioning of the hands/feet was deemed more likely. So this rule was instituted to further reduce measurement error. The drawback is of course that this rule enhances the possibility of overestimation of progression (bias) and that healing of erosions could not occur. As the rule was not applied for the chronological LS method, here improvement of scores could occur. However, in our set of films this was a rare phenomenon: in only $\|$ set of films improvement was abserved by both Larsen readers. One may therefore conclude that the influence of the rule on our results probably was negligible. Finally, the fact that the chronological SvH method in this study was applied with the rule that the scores 
can not decrease, does not imply that we don't believe that healing cannot occur. However, we believe that regarding the assessment of healing, scores should not simply be subtracted.

Our panel was made up of clinicians from different continents to ensure generalizability of the results. The intropanel agreement on clinically relevant changes was satisfactary, as appears from a kappa value of 0.59 . The intrapanel agreement on progression alone, irrespective of its clinical relevance, on the other hand, was low (0.45) in a setting of a high level of observed agreement $(0.85)$. The paradox of "high observed agreement and low kappa" is a well-known feature of kappa statistics and is caused by the fact that kappas are affected by the prevalence of the disease or condition concerned. ${ }^{14,15}$ Very low or high disease prevalence can result in misleadingly low kappas despite good agreement.

The film sets used we used were selected for high and low baseline scores and for high and low progression scores to reflect the spectrum of damage found in early RA trials. Our data selection was, however, restricted in that it did not include many film sets with major progression of joint damage. The sample, although rather small, was large enough to detect differences between the chronological and paired progression scores of the SuH method. However, the difference in sensitivity of the LS reading orders, also in favor of the chronological reading order, did not reach statistical significance in our rather small sample.

We used the opinion of an expert panel on the clinical relevance of progression in a patient with recent onset $\mathbb{R A}$ and high disease activity as external criterion. However, the influence of progression of radiolagical joint damage on the rheumatologists" treatment strategies could have differed if the disease duration would have been longer or if the patient would have had only mild disease activity. In our previous study, which involved the same panel members, the clinical importance of radiological joint progression was also assessed for 4 different clinical settings (early vs. late RA and mild ws. high disease activity). In that study, the level of progression scores with the highest accuracy indeed varied somewhat per setting: the panel was more likely to judge progression relevant for patients with early disease and high disease activity than for patients with late RA and mild disease activity, with the other 2 settings fitting in between. However, these highest accuracy progression scores also remained smaller than the SDD throughout, leading to similar conclusions for the different settings.

In this study, dinically important progression was defined as that progression in radiological joint damage that makes the rheumatologist change therapy. This decision of course also depends an other factors such as patient"s history of toxic reactions or availability of alternative treatment (such as tumor necrosis factor- $\alpha$ inhibitors). However, the panel was explicitly instructed not to include these factors. We asked them to state only their intention to change treatment, without taking into account other factors except the radiological progression of joint damage and the specification of the setting given. From the results of the previous study it was also clear that their judgments were indeed based on what they saw on the films and not merely on the clinical description. 
In conclusion, our study confirms greater sensitivity in detecting differences by scoring films in chronological order. These differences detected were defined as clinically relevant by an international expert panel. Therefore, knowing the sequence of films did not lead to overestimation of nonrelevant differences, but enabled better detection of clinically relevant changes. Scoring films without knowing the chronological sequence of the films substantially decreased the sensitivity in detection of clinically relevant changes as defined by an expert panel in comparison with scoring films in chronological order. In clinical trials of early RA, our results stronglly suggest that radiographs should be read in chronological order. 


\section{References}

1. Fries JF, Bloch DA, Sharp JT, McShane DJ, Spitz P, Bluhm GB, Forrester D, Genant $H$, Gotton $P$, Richman $S$, et al. Assessment of radiologic progression in rheumatoid arthritis. A randomized, controlled rial. Arthritis Rheum 1986;29:1.9.

2. Ferrara R, Priolo F, Cammisa M, Bacarini L, Cerase A, Pasero G, Ferraccioli GF, Alberighi OD, Antonellini A, Marubini E. Clinical Trials In Rheumatoid Arthritis: Methodological Suggestions For Assessing Radiographs Arising From the Grisar Study. Ann Rheum Dis 1997;56:008-12.

3. Salaffi F, Carotti M. Interobserver Variation in Quantitative Analysis Of Hand Radiographs in Rheumatoid Arthritis: Comparison of 3 Different Reading Procedures. J Rheumatol 1997;24:2055-56.

4. wan der Heilde D, Boonen A, Boers $M$, Kostense P, van Der Linden S. Reading radiographs in chronological order, in pairs or as single films has important implications for the discriminative power of rheumatoid arthritis clinical trials. Rheumatology (Oxford) 1999;38:1213-20.

5. van der Heijde D. How to read radiographs according to the Sharp/van der Helide method. I Rheumatol 2000;27:261-63.

6. Scoft DL, Houssien DA, Laasonen L. Proposed modification to Larsen's scoring methods for hand and wrist radiographs. $\mathrm{Br} J$ Rheumatol 1995;34:56.

7. Bruynesteyn $K$, van Der Heijde $D$, Boers $M$, Saudan $A$, Peloso P, Paulus $H$, Houben $H$, Griffiths $B$, Edmonds $J$, Bresnihan B, Boonen $A$, van der Linden S. Determination of the minimal clinically important difference in rheumatoid arthritis joint damage of the Sharp/van der Heijde and Larsen/Scott scoring methods by clinical experts and comparison with the smallest detectable difference. Arthritis Rheum 2002;46: 913-20.

8. van der Heijde DM. Plain X-rays in rheumatoid arthritis: overview of scoring methods, their reliability and applicability. Baillieres Clin Rheumatol 1996;10: 435-53.

9. Metz CE. Basic principles of ROC anolysis. Semin Nud Med 1978;8:283-98.

10. Bland $\mathrm{M}_{*}$. Atman DG. Statistical methods for assessing agreement between two methods of clinical measurement, Lancet 1986;1:307. 10.

1. Bland IM, Aliman DG. Measurement error. BMJ 1996;313:744.

12. Lassere M, Boer's M, van der Heijde D, Boonen A, Edmonds J, Saudan A, Verhoeven AC. Smallest detectable difference in radiological progression. J Rheumatol $1999: 26: 731 \cdot 39$.

13. Gardner $M_{s}$ Alman D. Statistics with confidence: confidence intervals and statical guidelimes. London: BMJ; 1989.

14. Lantz CA, Nebenzaht E. Behavior and interpretation of the kappa statistic: resolution of the two paradoxes. J Clin Epidemiol 1996;49:431-44.

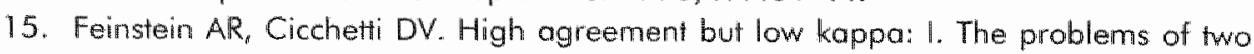
paradoxes. J Clin Epidemial 1990;43:543-49. 


\section{Chapter 7}

Deciding on progression of joint damage in paired films of individual patients: smallest defectable difference or change?

Bruynesteyn k. Boers M, Kostense P. won der Linden Sil wan der Heide D

Submitted for publication 


\section{Abstract}

To improve interpretation of study results these are often expressed as the proportion of patients meeting a certain target that is deemed clinically relevant. In studies of joint damage in theumatoid arthritis (RA), no such target has been defined; most agree the decision whether an individual patient has truly progressed should be based on a cutoff point that has a meaningful relationship with the measurement error of the instrument used to quantify the joint damage. As a sfarting point the smallest defectable difference (SDD) has been advised as cutoff level. The SDD expresses the smallest difference between 2 independently obtained scores that can be interpreted as "real", i.e., a difference beyond measurement error. However, radiological joint damage due to RA is usually assessed with the films of one particular patient side by side, i.e., simultaneously, with or without knowledge of the order of the films (chronological or paired reading, respectively.]. As a consequence, the change in scores is not based on 2 independently obtained scones.

We explain why the cutoff in this setring should be based on the smallest change in scores beyond measurement error (from here called the Smallest Detectable change, SDC) directly derived from the change-scores. As this report shows, the SDC is smaller than the SDD if films are judged side by side. 


\section{Introduction}

In therapeutic trials the main interest is usually the analysis based on the mean or median change of the outcome measure(s) over time. Results based on the number of patients responding to a particular intervention are, however, included increasingly in trial analyses because they can add valuable information. This is also true for the interpretation of radiological progression of joint damage due to rheumatoid arthritis (RA) in the hands and feet." Radiographic data show a highly skewed distribution pattern: the majority of patients show only mild or no pragression in the observation period, and only a subset of patients show substantial progression. In this situation presenting the percentage of patients with progression gives additional insight into the data compared to the mean and median. Furthermore, by determining the number of patients responding to the intervention under investigation, one is able to assess the number needed to treat (NNT), which enables clinicians to translate the results from trials to clinical practice.

To determine the percentage of patients that showed a relevant change over time a minimum clinically important difference is required to determine a cutoff level. Mast agree that such a farget, or cutoff value should at least be over the measurement error of the instrument used to quantify the response. Note that in the setting of damage progression, "response" can be translated to no progression (and theoretically, improvement or repair). As a starting point the so-called smallest detectable difference (SDD) has therefore been advised as cutoff level., 1,4 The SDD expresses the smallest difference between 2 independently obtained results (scores) that can be interpreted as "real" "i.e., a difference beyond measurement error. However, radiological joint damage due to RA is usually assessed with the fillms of one particular patient side by side, i.e., simultaneously, with or without knowledge of the order of the films (chronological or paired reading, respectively). In this way raters can compensate for variation in positioning of the hands and feet and in film quality. As a consequence, the regular methods used to assess the smallest changes in scores that can be deemed as "real" changes (SDD) are inappropriate.

Aim of this paper is to present how to assess a smallest detectable change (from here on called the SDC) in the setting of films read simultaneously. Further we will demonstrate that the SDD is larger than the SDC, i.e., overestimates the measurement error in this setting.

In the next paragraphs, we will first further explain why the regular methods by calculating SDDs should not be used in its current format to assess a cutoff for a change in an individual patient. Then, we will describe two methods to correctly estimate the measurement error and calculate a SDC. Finally, we will give an example of both calculations based on the reliability data of 10 random subjects of the COBRA trial ${ }^{5}$ and also demonstrate that if films are not scored 
independenily, the SDD can overestimate the measurement error of detecting change. For simplicity, we will assume a setting of one baseline and one follow up measurement, although the principle applies equally to multiple follow-up measurements.

\section{Random reading versus paired reading}

Whether a change in radiological joint damage is based on 2 independently abtained scores or not depends on how the radiographs are presented to the rater. Radiographs can be presented to a rater completely at random, i.e., a single film at a time, or they can be grouped per patient so that all films of one particular patient are read simultaneously, with or withoul data on the sequence of the films. Reading all films of one patient simultaneously, has as major advantage that the rater will be able to carrect for variation in positioning of the hands and feet or variation of the film quality. When films are grouped per patient, a rater compares all films of one patient and judges whether a change in joint damage has occurred. Paired (without information on the chronology of the films) or sequenced (with information on the chronology of the films) reading thereby aims to reduce the measurement error of the object of interest in trials: the change in joint damage. However, the measurement error of the scores of the patients are hereby correlated and are no longer independent. Furthermore, the change-score (the score obtained by subtracting the status score at time 1 from the status score at time 2) can actually be interpreted as a single score. In order to determine the SDC in the situation that films are read simultaneously, the hypothesis to be tested is whether the change-score in an individual patient is different from zero. When determining the "regular" SDD the hypothesis tested is whether the difference between 2 independent status scores is different from zero. ${ }^{2,4,6,7}$ Finally, when determining a SDD based on the change-score, the hypothesis tested is whether the difference between 2 independent change scores is different from zero. So the SDD based on change-scores is the correct measure if we want to test whether the changes-score from one patient is different from the change-score of another patient. In case we want to assess if an individual patient shows progression, the SDC is more appropriate.

Note that with independent scores we do not refer to the condition that scores of one person are independent statistically. Scores of one patient are always paired observations and thereby statistically dependent. With independent scores we reffer to the fact that the measurement error of the scores of the films from time point 2 is not related to the measurement error of the scores of the films from time point 1 . When scoring films of time point 1 and 2 simultaneously this assumption is violated.

Appropriate methods to assess the SDC for paired or sequenced reading of films

In the field of radiological joint damage, it is customary to assess the measurement error of the scoring methods by re-scoring the radiographs by the same rater (intra-rater reliability) or by a different rater (inter-rater reliability). The option of making a second radiograph is never applied on a large scale in 
the context of trials because this implies additional exposure to radiation of the patients. Because the same radiographs are re-examined, differences between the 2 observations on each individual are caused by measurement error. To assess a SDC one needs to determine the error of the scoring method when measuring a change in joint damage. We shall describe 2 methods to estimate the measurement error of the change. The first method quantifies measurement error by the Standard Error of Measurement (SEMchange-scare) derived from a twoway analysis of variance (ANOVA) in which the change-scores of 2 reading sessions are analysed. The second method estimates the measurement error by calculating the standard deviation of the difference between change-scores of 2 reading sessions (SD $\Delta$ (chronge-score)). The latter resembles the estimation of the measurement error as described by Bland and Altman ${ }^{3,6}$, in which the standard deviation of the difference between status scores of 2 reading sessions [SDAstratus. scorel) estimates the measurement error. The latter is only applicable for 2 raters or 1 repetition; the first method can be applied also if measurements of 3 or more raters or 2 or more repetitions are available.

\section{Measurement error estimated with the SEM CHANGE-SCORE}

With a two-way ANOVA based on change-scores of repeated measurements or change-scores of 2 or more raters one can estimate the measurement error from the results of simple reliability studies, in which one source of measurement error at a time is determined. The two-way ANOVA will result in mean squares (MS) for the different sources of variation: the between-patients variation and the within-patients variation; the latter being composed of between-measurements variation and the residual error. A SEM expresses the amount of measurement error in the original metric unit of the measurement and is derived by taking the square root of the error variance. The error variance may constitute the tatal within-patient variance (including the between-measurements variance) or the residual variance only. If one does not want to generalize to other raters not included in the reliability study, the between-measurements variance (i.e., the variance due to differences between the scores of the raters) should be not included and the error variance then only includes the residual variance (variance due to the unknown sources). To be able to compare the SDC calculated with a SEMchange-scare directly with the SDC calculated with the SDLichange-score, the SEMchange-score will be based in this paper on the residual error. Because a SEM is a variability quantity it can be used to callculate an interval of error around scores, under the assumption that the measurement error is distributed normally. At the $95 \%$ confidence level, the interval around a change-score is calculated according to the formula [change-score $\pm 1.96^{*}$ SEMachange-score]. If in the actual study the measurement is repeated (e.g., 2 raters reading all films independently) and the mean of both readings is to be used in the analyses, the measurement error diminishes by a factor $\sqrt{k}$ where $k$ represents the number of readings. The formula then becomes [change-score $\pm 1.96^{*}$ SEMchange-score/ $\sqrt{k}$ ]. In the following we will routinely be using the formula that includes $\sqrt{k}$ in the denominator. If the interval [change-score $\pm 1.96^{*}$ SEMchange-score/ $\sqrt{k}$ ] contains the value zero, the null hypothesis that the 
change-score is zero cannot be rejected. So, values of the change-score lying in this interval other then zero could be induced by error alone. On the other hand, if the interval does not include zero we reject the null hypothesis and conclude that the change-score is really different from zero and state that there is a "real" change in joint damage. Note that the absolute change (i.e., disregarding the sign) needs only to be half the size of the full interval described by [change-score $\pm 1.96^{*}$ SEMchange-score/ $\left.\sqrt{k}\right]$. In other words: in the case of two readings the first can be either larger or smaller than the second purelly by measurement error, and the full range of possible changes (with positive and negative sign) is described by the interval in the formula. However, once we find the first reading to be larger than the second we will reject the null hypothesis ance the change falls outside the range of the interval, in this case on the positive side. This will occur when the change is half the size of the full interval. To conclude, the formula for the SDC based on the SEMchange-scare is: $\left[ \pm 1.96^{*}\right.$ SEMchonge-score/ $\left.\sqrt{k}\right]$.

2. Measurement error estimated with the SD of the differences between changescores of 2 reading sessions ( $S D_{\Delta \text { (change-score)). }}$ ).

If intra-rater data of repeated measurements or inter-rater data of 2 raters are used to assess the SDC, the measurement error can also be estimated with the standard deviation of the differences between change-scores of 2 reading sessions (SDAichange-score)). For this method one first calculates the differences between the change-scores obtained in the repeated reading session. Second, the SD of these differences is calculated. This SDaichange-score) reflects the measurement error of the difference between 2 change-scores, i.e., the measurement error when discriminating between 2 change-scores. However for the SDC one is interested whether a single change-score really can be distinguished form zero and not whether 2 change-scores are different from each other. The measurement error of a single change-score is abtained by

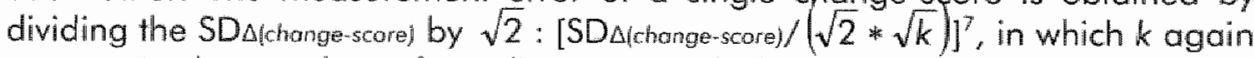
represents the number of readings over which one wants to average. The formula [SDAtchange-scare) $/(\sqrt{2} * \sqrt{k})$ ] equals the formula [SEMchonge-score/ $\sqrt{k}$ ] if the data of one repetition or 2 raters is used. ${ }^{8}$ The error interval around a changescore, at the $95 \%$ confidence level, is consequently calculated by [chonge-score $\pm 1.96^{*} \mathrm{SD}$ sichonge-scare $/(\sqrt{2} * \sqrt{k})$, and the SDC is calculated by $\left[ \pm 1.96^{*} \mathrm{SD} \Delta[\right.$ change-score $/(\sqrt{2} * \sqrt{k})]$, similar to the calculations based on the SEMtchange-scare.

In the next section we'll show both calculations for the reliability data of 10 subjects form the COBRA trial." Radialogical data is however often highly skewed and because it is known that measurement error tends to be larger in studies with more baseline damage and radiological progression ${ }^{3}$, the assumption of homoscedasticity can be violated. A Bland and Altman plot (plotting the difference of the raters scores and the mean of the raters' scores) of the COBRA data showed homoscedasticity in subjects with a progression score of $<25$ Sharp/van der Heijde units $\left(90^{\text {th }}\right.$ percentile), so the 10 subjects were randomly selected from this subgroup. 
Example of the 2 methods to assess the SDC for scores based on paired or sequenced reading of films.

Table 7.1 shows the scores of 2 different raters of the 10 subjects. The SDQtchange-scores in this example is 2.06 and calculating the SDC with the formula $1.96 * 2.06 /(\sqrt{2} * \sqrt{k})$ results in a SDC of 2.85 scoring units if not using average scores $(k=1)$.

TABLE 7.1

Scores of 2 different raters of 10 patients

\begin{tabular}{|c|c|c|c|c|c|c|}
\hline Subject & $\begin{array}{c}\text { Baseline } \\
\text { score } \\
\text { lst rater }\end{array}$ & $\begin{array}{l}\text { Baseline } \\
\text { score } \\
2 \text { ndirater }\end{array}$ & $\begin{array}{l}\text { Difference } \\
\text { status-scorres } \\
\text { between raters }\end{array}$ & $\begin{array}{l}\text { Change } \\
\text { score Ist } \\
\text { rater }\end{array}$ & $\begin{array}{c}\text { Change } \\
\text { score } 2 \text { nd } \\
\text { rater }\end{array}$ & $\begin{array}{l}\text { Difference } \\
\text { change-scores } \\
\text { behween raters }\end{array}$ \\
\hline 1 & 12 & 15 & 3 & 2 & 3 & 1 \\
\hline 2 & 0 & 0 & 0 & 0 & 0 & 0 \\
\hline 3 & 23 & 20 & -3 & 9 & 10 & 1 \\
\hline 4 & 5 & 2 & -3 & 4 & 1 & -3 \\
\hline 5 & 7 & 11 & 4 & 11 & 10 & -1 \\
\hline 6 & 3 & 0 & -3 & 1 & 0 & -1 \\
\hline 7 & 0 & 0 & 0 & 0 & 0 & 0 \\
\hline 8 & 1 & 1 & 0 & 5 & 8 & 3 \\
\hline 9 & 9 & 6 & -3 & 17 & 13 & -4 \\
\hline 10 & 19 & 25 & 6 & 10 & 11 & 1 \\
\hline Mean & & & 0.1 & & & -0.3 \\
\hline SD & & & 3.28 & & & 2.06 \\
\hline
\end{tabular}

The ANOVA for the change-score data of Table 7.1 is presented in Table 7.2. The SEMchange-score is calculated by extrading the root of the residual mean square $(\sqrt{2.12}=1.46)$. The SDC is then calculated by $1.96 *(1.46 / \sqrt{k})$, which again results in 2.85 scoring units if not using average scores $(k=1)$.

Overestimation of the measurement error by the SDD

To complete this report, we show that basing the cutoff level for analyses on the individual level on the SDD between 2 status scores will results in an overestimation of the SDC when films are read simultaneously. The SDD according to Bland and Altman ${ }^{3,6}$ is estimated by calculating a $95 \%$ interval around the difference between 2 single status scores. This cam be done under the assumption that the wariances of the measurement error of both status scores are identical and independent, and as a consequence the variance of the difference is twice that of the single measurement error; varsistatus-scors $)=2$ yarmeasurement error. To estimate the measurement error of the difference between paired status scores again a two-way ANOVA can be used or the SD of the differences between the status scores. 
TABLE 7.2

Two-way analysis of variance for the change-score data of Table 7.1

\begin{tabular}{lccc} 
Source of variafion & Degrees of freedom & Sum of Squares & Mean Square \\
\hline Between-patients" & 9 & 520.25 & 57.81 \\
Within-patients $^{t}$ & 10 & 19.50 & 1.95 \\
Between-measurements $^{*}$ & 1 & 0.45 & 0.45 \\
Residual & 9 & 19.05 & 2.12 \\
Total & 19 & 539.75 & \\
\hline
\end{tabular}

- wariance in scores due to differences between patients, t variance in scores due to differences within a patient, 1 variance in cores due to the differences between the raters, $f$ variance in scores due to unknown sounces

The SEM of the difference is the square root of the variance of the difference: SEMA(statius-score) $=\sqrt{W A R}$ status-scare $=\sqrt{2} *$ SEM status-score. The SDD is consequently calculated by the formula $\pm 1.96^{*} \sqrt{2} *$ (SEMstorus-score/ $\sqrt{k}$ ) or by $\pm 1.96^{*}$ (SD (statusscores)/ $\sqrt{k}$ ) ( $\sqrt{k}$ included in the denominator as before). Table 7.3 shows the baseline scores of the 2 raters. Calculating the SDD with the SDAistatus-score) $( \pm 1.96 *(3.28 /(\sqrt{k}))$ or with the SEMstatus-scare $( \pm 1.96 * \sqrt{2} *(\sqrt{5.38} / \sqrt{k}))$ results a SDD of 6.4 units if not using average scores $(k=1)$. So, the measurement error of detecting a change within patients is clearly smaller than the measurement error of detecting a difference between 2 single baseline scores, as expected.

In summary, progression of radiological joint damage is usually based on simultaneous assessment of a series of films from an individual patient ("paired", with or without known sequencel. In this setting, the amount of progression that is reliably detectable above measurement error is best determined by the smallest detectable change, and overestimated by the traditionally (but inappropriately) calculated smallest detectable difference.

TABLE 7.3

Twa-way analysis of variance for the baseline status score data of Table 7.1

\begin{tabular}{lccc} 
Source of voriation & Degrees of freedom & Sum of Squares & Mean Square \\
Between-patients & 9 & 1298.45 & 144.27 \\
Within patients $^{t}$ & 10 & 48.5 & 4.85 \\
Benween-measurements $^{*}$ & 1 & 0.05 & 0.05 \\
Residual & 9 & 48.45 & 5.38 \\
Total & 19 & 1346.95 & \\
\hline
\end{tabular}

Mariance in scores due to differences between patients, variance in scores due to differences within a paitient? variance in scares due lo the differences behween the roters; Gorionce in scores due to unknown sources 


\section{References}

1. van der Heijde D, Simon L, Smolen J, Strand V, Sharp 1, Boers M, Breedweld F, Weisman $M$, Weinblatt $M$, Rau $R$, Lipsky $P$. How to report radiographic data in randomized dinical trials in rheumatoid anthritis: guidelines from a roundtable discussion. Arthritis Rheum 2002;47:215.8.

2. Streiner $\mathbb{D L}$, Norman GR. Measuring change. Health measurements scales. $A$ practical guide to their development and use: Oxford University Press, 1995:16380.

3. Lassere $M_{*}$ Boers $M_{*}$ van der Heilde D, Boonen A, Edmonds J, Saudan $A$, Verhoeven AC. Smallest defectable difference in radiological progression. I Rheumatal 1999;26:731-9.

4. Ravaud P, Giraudeau B, Auleley GR, Edouard-Noel R, Dougados M, Chastang C. Assessing smallest detectable change over time in confinuous structural outcome measures: application to radialogical change in knee osteaarthritis. J Cin Epidemial $1999,52: 1225-30$.

5. Boers $M$, Verhoeven $A C$, Markusse $H M$, van de Laar MA, Westhovens $R$, van Denderen JC, van Zeben D, Dijkmans BA, Peeters AJ, Jacobs $P$, wan den Brink HR, Schouten HJ, van der Helije DM, Boanen A, van der Linden S. Randomised comparison of combined step-down prednisolone, methotrexate and sulphasalazine with sulphasalazine alone in early rheumatoid arthritis. Lancet 1997,350:309-18.

6. Bland JM. Altman DG. Statistical methods for assessing agreement bewween two methods of clinicall measurement. Lancet 1986;1:307.10.

7. Beckerman H, Roebroeck ME, Lankhorst GJ, Becher JG, Bezemer PD, Verbeek AL. Smallest real difference, a link between reproducibility and responsiveness. Qual Life Res 2001;10:571-8.

8. Hopkins WG. Measures of reliability in sports medicine and science. Sports Med $2000 ; 30: 1-15$. 


\section{Chapter 8}

How the type of risk reduction influences required sample sizes in randomised clinical trials

Bruynesteyn K, Wanders A, Landewé R, van der Heijde $\mathrm{D}$

Submitted for publication 


\section{Abstract}

In order to increase chonge (and treatment contrast) between groups, randomised clinical trials (RCT) often include patients with a high risk on a particular outcome, by inclusion eriteria that select on predictors for that outcome. The general wiew is that such a strategy increases the statistical power, and thus limits the number of patients required for that RCT.

How the selection of patients influences the power and thus the sample size required, depends on how an intervention reduces the individual risk: by an absolute or relative risk reduction model (ARR or RRR-model, respectively). We here explain both madels and show that if a treatment mainly acts according to the RRR-model, selection of patients with a high prior risk will result in higher statistical power, and thus smaller sample sizes. If a trealment acts mainly according to the ARR-model, selection of patients by priar risk may have divergent effects on the sample size required in trials with dichotomaus outcome measures: statistical power is worst in case of a prior risk of on average $-50 \%$, which can be the case when including a mixture of high, intermediate and low risk patients. As a consequence, the required sample size will be higher.

To explore the relationship between prior risk and treatment effect in rheumatological frials, we analysed the data of two trials in which disease modifying anti-rheumatic drugs (DMARDs) in rheumatoid arthritis (RA) were inwestigated. Radiological progression (above or below the median) was defined as outicome, and joint damage at baseline (present or absent) as prior risk. In both trials, the DMARD treatment with highest efficacy seemed to act mainly according to the ARR-model.

In order to design appropriately powered RCTs with dichotomousoutcome measures, it is relevant to be informed about whether tested therapies work mainly according to a RRR or according to an ARR-model. If data are lacking, it is best to select patients with a high prior risk on the outcome that should be prevented by the therapy, under the assumption that this is feasible. 
In order to increase the contrast between an intervention and a contral group, randomised clinical frials (RCTs) often include patients with a high prior risk on the outcome of interest by selecting on baseline predictors for that outcome. The general view is that such a strategy limits the number of patienis required, by increasing the statistical power. In RCTs in RA, one important outcome is progression of radiolagical damage. It is known that joint damage present at baseline (prior risk) is one of the strongest predictors for futher progression of radiological joint damage. ${ }^{1.4}$ Therefore, we expected fewer palients would be needed for a RCT if patients were selected for the presence of joint damage at baseline. Somewhat surprisingly, power calculations learned that this expectation was false. To elucidate this counterintuitive observation, we performed a literature search, and we found that statistical power is determined by a critical relationship between the prior risk on a particular dichotomous outcome (this is the baseline risk, independent of treatment), and on how al particular treatment exerts its efficacy with respect to this prior risk. We will explain this relationship step-by-step, and illustrate it with hypothetical and authentic data.

The risk of an individual patient on a particular outcome can be reduced in two different ways, according to the following two models: the absolute risk reduction model, and the relative risk reduction model. For understanding these models it is necessary to be familiar with the terms absolute risk reduction (ARR) and relative risk reduction (RRR).

Consider a RCT comparing an active treatment with placebo. The autcome of interest is binomial (event or no event) and the active treatment can reduce the probability of that event. So, the event rate in the treatment group $\left(\mathbb{P}_{t}\right)$ is supposed to be lower than the event rate in the placebo group $\left(\mathbb{P}_{\mathrm{p}}\right)$. Absolute risk reduction ( $A R R$ ) is the difference in the event rates between the placebo and the treatment group $\left(P_{p}-P_{t}\right)$. Relative risk is defined here as the ratio of the event rate in the treatment group and that in the placebo group $\left(P_{t} / P_{p}\right)$. RRR is defined as the reduction of the event rate in the treatment group, in proportion to that in the placebo group, or: $\left(\mathrm{P}_{\mathrm{p}}+\mathrm{P}_{\mathrm{H}}\right) / \mathrm{P}_{\mathrm{p} \text {. }}$ This is mathematically similar to $(1$ - the relative risk), or: $\left(1-P_{2} / P_{p}\right) . R R R$ is usually expressed as a percentage, or: (1. $\left.\left(P_{i} / P_{p}\right) * 100 \%\right)$

If a therapy works according to a RRR-model, the RRR remains constant, irrespective of the prior risk, whereas the ARR varies with the prior risk. If a therapy works according to an ARR-model, the ARR remains constant for all patients irrespective of prior risk and then the RRR varies with different prior risks.

Suppose that we distinguish three subgroups, defined by the prior risk on a particular outcome: one group with a low prior risk, one group with an intermediate prior risk, and one group with a high prior risk. Nate that prior risk does not directly refer to the event rate in the control group, but rather to patient characteristics (such as age, gender, disease aetiology, concomitant conditions or disease status) measured at baseline, and known for their ability to influence the probability of the negative outcome. Suppose also that without any 
(adequate) treatment the negative outcome will occur in $20 \%, 60 \%$ and $90 \%$ of the patients per group, respectively. Assume that therapy works purely according to the RRR-model (Table 8.1), providing a RRR of $50 \%$. So, treatment will lower the event rate from $20 \%$ to $10 \%$ (50\% reduction) in the low risk group, from $60 \%$ to $30 \%$ (50\% reduction) in the intermediate risk group, and from $90 \%$ to $45 \%$ (50\% reduction) in the high-risk group. The ARR is now $10 \%, 30 \%$, and $45 \%$ respectively. These figures can be used to calculate estimated sample sizes for future clinical trials. We calculated the samples sizes for two-sided statistical testing using the power calculator of the UCLA department of statistics with the 2-sample arcsine approximation of the binomial distribution, with alpha set at 0.05 and beta at $0.20 .^{5}$ From Table 8.1 (sample sizes per group) it is obvious that the baseline risk importantly influences the appropriate sample size. In other words: In this scenario, selecting high-risk patients will increase the statistical power of a trial.

TABLE 8.1

Example of the relative risk reduction model

\begin{tabular}{|c|c|c|c|c|c|}
\hline Risk graups & $\begin{array}{l}\text { Negotiy } \\
\text { Control }\end{array}$ & $\begin{array}{l}\text { eventrate } \\
\text { Treamment }\end{array}$ & RRR & ARR & $\begin{array}{l}\text { Sample size } \\
\text { per group }\end{array}$ \\
\hline Low baseline risk & $20 \%$ & $10 \%$ & $50 \%$ & $10 \%$ & 195 \\
\hline Medium baseline risk & $60 \%$ & $30 \%$ & $50 \%$ & $30 \%$ & 41 \\
\hline High baseline risk & $90 \%$ & $45 \%$ & $50 \%$ & $45 \%$ & 14 \\
\hline
\end{tabular}

$\mathrm{RRR}=1$ - outconne rate treattment group divided by outcome rate controls] * 100], ARR - [outcome rote contral group - outcome hote treatment groupl, Sample size calculotion based on alpha $=0.05$, beta 020 , wo sided.

Now assume that a therapy works purely according to the ARR-model (Table 8.2 ), providing an ARR of $10 \%$. Based on the same prior risk percentages, treatment will lower the event rate from 20\% to $10 \%(10 \%$ reduction) in the low risk group, from $60 \%$ to $50 \%$ (10\% reduction) in the intermediate risk group, and from $90 \%$ to $80 \%(10 \%$ reduction) in the high-risk group. The RRR is now $50 \%\left((1-(10 \% / 20 \%))^{*} 100\right)$ in the low risk group, $17 \%\left((1-(50 \% / 60 \%))^{*} 100\right)$ in the intermediate risk group and $11 \%\left((1-(80 \% / 90 \%))^{*} 100\right)$ in the high-risk group, respectively. If again sample sizes are estimated using these figures, it becomes clear that the same sample sizes are required in low and high-risk groups to statistically demonstrate an ARR of 10\%. But to demonstrate an ARR of $10 \%$ for the intermediate risk group, a much larger sample size is needed. This can be explained by the fact that for intermediate risk groups the probability on the occurrence of a negative outcome is almost similar to the probability on the occurrence of a positive outcome. Note that an intermediate risk group may either include patients with an intermediate prior risk only, or a mix of patients with a low, high and intermediate risk. 
Summarized: depending on how a therapy reduces the individual's risk on a particular negative outcome (according to an ARR - or G RRR-modell, the patient selection for the trial with respect to the prior risk may influence the statistical power, and accordingly the sample size required for that trial. If a theropy (mainly) acts according to the RRR- model, trials including patients with prognostic variables for a negative outcome (high prior risk) will yield mare statistical power, and require a lower sample size. If a therapy acts mainly according to the ARR- model, selection of patients with respect to prognostic variables has a different effect on sample size: From a statistical point of wiew, it would be wise to avoid groups of patients with an average prior risk on the negative outcome of approximately $50 \%$. Trials with such patient groups provide less statistical power, as compared to trials with patient groups with a low or a high prior risk.

\section{TABLE 8.2}

Example of the absolute risk reduction madel

\begin{tabular}{|c|c|c|c|c|c|}
\hline \multirow{2}{*}{ Risk groups } & \multicolumn{2}{|c|}{ Negalive event rate } & \multirow[t]{2}{*}{ RRR } & \multirow[t]{2}{*}{ ARR } & \multirow{2}{*}{$\begin{array}{l}\text { Sample size } \\
\text { per group }\end{array}$} \\
\hline & Control & Treatment & & & \\
\hline Low baseline risk & $20 \%$ & $10 \%$ & $50 \%$ & $10 \%$ & 195 \\
\hline Medium baseline risk & $60 \%$ & $50 \%$ & $17 \%$ & $10 \%$ & 387 \\
\hline High baseline risk & $90 \%$ & $80 \%$ & $11 \%$ & $10 \%$ & 195 \\
\hline
\end{tabular}

$\mathrm{RRR}=[1$ (outcome raite treatmenl group civided by outcame rate controls) * 100, ARR = [outcome rale control group - outcome rarte treatmend groupl Sample size calculation based on alpha $=0.05$, beta $=0.20$, wo sided

In the sparse literature that evaluated the relationship between treatment effect and the prior risk, it is suggested that the RRR is constant across the usual spectrum of prior risks in the vast majority of treatments (i.e., following the RRRmodell.6.7 Nevertheless exceptions have been described. A large study evaluating the stroke risk in patients treated with aspirin, showed a decreasing RRR by increasing prior risk. However, the ARR also decreased by increasing baseline risk, so actually a mix of both models seemed to be operative. Obviously, treatments do not always act strictly according the RRR- or to the ARR model: Mixed models can also be found.

To our best knowledge, in theumatology no research has been performed to examine the relationship between prior risks and models of risk reduction, with respect to treatment effect. Recently, radiographic data of a RCT comparing methotrexate (MTX) + cyclosporine (CsA) versus CsA monotherapy have become available ${ }^{10}$. We used these data to investigate this relationship. Treatment effect was defined here as the reduction in the proportion of patients with the negative 
outcome, being radiagraphic progression $\geq$ the median group level, at one year. The prior risk on radiographic progression (baseline risk) was based on radiological damage at baseline above or beneath the median. Table 8.3 shows that the RRR was not similar in the two baseline risk groups $150 \%$ in the intermediate risk group vs. $37 \%$ in the high-risk group): The addition of MTX to CSA appeared not to follow a pure RRR model, but rather an ARR model (the ARR was approximately similar in both risk groups (25\% vs. $29 \%)$ ).

We further explored the radiographic data of the COBRA trial (Table 8.4), in order to confirm the phenomenon of a decreasing RRR by an increasing baseline risk. In the COBRA trial', combination treatment with step-down prednisolone, MTX and sulfasalazine (SSZ) was compared with SSZ monotherapy. We again defined treatment effect as the reduction in patients with radiographic progression above the median levell in one year.

Table 8.4 shows that the ARR (combination therapy, compared with SSZ alone) was almost similar in the two risk groups $(22 \%$ vs. $25 \%)$, and that the RRR decreased by increasing baseline risk ( $54 \%$ vs. $30 \%)$.

TABLE 8.3

CSA + MTX compared with CSA alone

\begin{tabular}{lccccc} 
Risk groups* $^{*}$ & Negative event rate* & RRR & ARR & Sample size \\
& CSA placebo & CSA+MT & & & per group \\
\hline Medium baseline risk & $50 \%(n=32)$ & $25 \%(n=26)$ & $50 \%$ & $25 \%$ & 57 \\
High baseline risk & $79 \%(n=28)$ & $50 \%(n=29)$ & $37 \%$ & $29 \%$ & 40 \\
\hline
\end{tabular}

$R R R=\left[1\right.$ - butcome rate treatment group divided by outcome rate cantrols] ${ }^{*}$ 109], ARR-[outcome rate control group - outcome rate treatment group] sample size calculation based on alphio $=0.05$, betol $=0.20$, two sided.

CSA = cyclosporine, MTX = methotrexate;

Risk groups based on baseline radiographic damage:

* Percentage of patients with radiographic progression larger than the median

We statistically compared the treatment effects in both subgroups of baseline damage, in order to test the null hypothesis that the relative risk reductions were similar (test of interaction, as recommended by Matthews \& Altman, and Altman \& Bland). ${ }^{11,12}$ In neither of both studies, a significant interaction could be demonstrated $(P=0.29$ for the difference in treatment effects in the MTX+CsA study, and $P=0.16$ for the difference in treatment effects in the COBRA study). For the test of interaction, as well as for an interpretation and discussion of the lack of statistical interaction, we refer to the literature..$^{11,12}$

These observations can of course not be generalised to the effect of all DMARDS on radiological joint damage. However, if it is true that some of the DMARDS decrease radiological progression by means of an ARR rather than a RRR madel, selection of patients with an average prior risk on radiological progression of 
about $50 \%$ should be avaided when a RCT with such DMARD is assigned (Table 8.2). But only a proportion of all patients with RA that eventually show radiological progression will have radiological joint damage at inclusion. This makes it difficult to selectively enrol patients with a high baseline risk, and the actual patient accrual may include patients with intermediate, rather than with high baseline risk.

\section{TABLE 8.4}

COBRA treatment compared with SSZ alone

\begin{tabular}{|c|c|c|c|c|c|}
\hline \multirow[t]{2}{*}{ Risk groups } & \multicolumn{2}{|c|}{ Negative event role* } & \multirow[t]{2}{*}{ RRR } & \multirow[t]{2}{*}{ ARR } & \multirow{2}{*}{$\begin{array}{l}\text { Sample size } \\
\text { per group }\end{array}$} \\
\hline & $S S Z$ & COBRA & & & \\
\hline Medium baseline risk & $40 \%(n=23)$ & $18 \%(n=34)$ & $54 \%$ & $22 \%$ & 68 \\
\hline High baseline risk & $82 \%(n=27)$ & $58 \%(n=27)$ & $30 \%$ & $25 \%$ & 51 \\
\hline
\end{tabular}

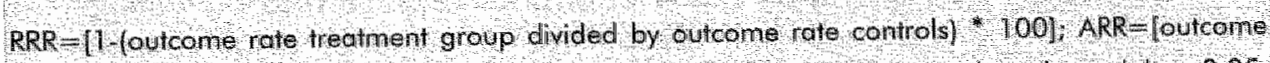
rate conhol graup - outcome rate herapy groupl, Sample size calculation based on alpha $=0.5$ betal $=020$ hwo sided.

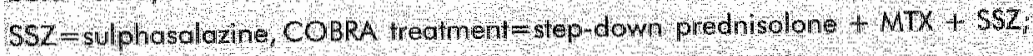

* Risk groups basod on baseline radiographic elamege;

* Pecentoge al patients wh rodiographic progression larger hich the hedion

We want to make a few additional remarks. First, a trialist who is developing a trial has to make a choice between aiming at a mixture of high-, intermediateand low-risk patients, and focussing on just one category. For generalizability purposes one may choose to include all types of risk patients. However, we showed here that this might lead to larger sample sizes. On the ather hand, ane should consider whether the preferred inclusion of high-risk patients is teasible. If high-risk patients are difficult to include for any reason, the argument of an appropriate recruitment rate may outweigh the argument of limited sample sizes. by the selective inclusion of high-risk patients.

Patient selection in RCTs is often based on characteristics that are predictive of a negative outcome. Aim of this report was partly to show that statistical power is dependent on the level of that prior risk, as well as on how treatment actually reduces that risk. This "is a different appraach as compared to selecting patients on the individual likelihood of responding to a particular treatment. Selecting patients with a high probability of responding to a particular treatment will also result in an increasing ARR. This means more statistical power, and as a consequence, a smaller required sample size. Increased responsiveness at a group level in RCTs can be promoted in wo different ways. "The first is by taking measures that result in a general increase of patient's compliance. Those who take their medicine (appropriately) might respond better than those who do not take their medicine (approprictely). The second way to promote 
responsiveness is by identifying subgroups of patients that are intrinsically responsive to the particular treatment. But patient characteristics that are predictive of a high response to any treatment have hardly be identified in rheumatology up to now. This will be an important research area in the future.

Our final remark refers to the models used to describe the relationship between the prior risk and the treatment effect. These models are based on discrete binomial outcome measures. The primary outcome measure of a trial can also be a continuous variable. Although the models cannot simply be translated to continuous measures, there are no arguments why treatment effects should act differently when measured on a continuous scale as compared to a dichotomous one.

We here conclude that patient selection with regard to factors predictive of the outcome that should be influenced by therapy has an impact on the statistical power of RCTs with dichotomous outcome measures. The precise direction of that impact depends not only on the level of prior risk, but also on whether the ARR or the RRR remains constant irrespective of the prior risk. As a rule of thumb, statistical power is best guaranteed by selecting high-risk patients, because this scenario omits the dependency of the type of risk reduction. Better insight in prediction of individual responsiveness may further increase statistical power and decrease required sample size. 


\section{References}

1. Uhing T, Smedstad LM, Vaglum P, Moum T, Gerard N, Kvien TK. The course of rheumatoid arthritis and predictors of psychological physical and radiographic outcome after 5 years of follow-up. Rheumatology (Oxford) $2000 ; 39: 732-741$.

2. Boers M, Kostense PJ, Verhoeven AC, van der Linden S. Inflammation and damage in an individual joint predict further damage in that joint in patients with early fheumatoid arthritis. Anthritis Rheun 2001;44:2242-6.

3. Combe B, Dougados M, Goupille P, Cantagrel A, Eliaou JF, Sibilia J, Meyer O, Sany 1. Daures IP, Dubois A. Prognostic factors for radiographic damage in early rheumatoid arthritis: a multiparameter prospective study. Arthritis Rheum $2001: 44: 1736-43$.

4. Drossaers-Bakker KW, Zwinderman AH, Wlieland TP, Van Zeben D, Vos K, Breedveld FC, Hazes JM. Long-term outcome in rheumatoid arthritis: a simple algorithm of baseline parameters can predict radiographic damage, disability, and disease course at 12-year followup. Arthritis Rheum 2002;47:383-90.

5. hitp://calculators.stat.uda.edu/powercale/

6. Osiri M, Suarez-Almazor ME, Wellis GA, Robinson V, Tugwell P. Number needed to treat (NNT): implication in rheumatology dinical practice. Ann Rheum Dis 2003, $62: 316-21$

7. McAlister FA. Commentary: relative treatment effects are consistent acrass the spectrum of underlying risks...usually. Int J Epidemiol 2002;31:76-7.

8. Rothwell PM. Can overall results of clinical trials be applied to all patients? Lancet 1995:345:1616-9.

9. Boers $M$, Verhoeven $A C$, Markusse $H M$, van de Laar $M$, Westhovens $R$, van Denderen JC, van Zeben D, Dijkmans BAC, Peeters AJ, Jacobs $P$, van den Brink HR, Schouten HJA, van der Heijde D, Boonen $A_{*}$ wan der Linden S. Randomised comparison of combined step-down prednisolone, methotrexate and sulphasalazine with sulphasalazine alone in early theumatoid arthritis. Lancet 1997;350:309-18.

10. Gerards AH, Landewé RBM, Prins APA, Bruijn GAW, Goei The HS, Laan RFM, Dijkmans BAC. Cyclosporin A monotherapy versus cyclosporin A and methotrexate combination therapy in patients with early theumatoid athritis: a double blind randomised placebo controlled trial. Ann Rheum Dis 2003, 62:291-96.

11. Matthews JN \& Altman DG. Statistical notes: Interaction 3: How to examine heterogeneity. BMJ 1996, 313:862.

12. Altman DG, Bland JM. Statistical notes: Interaction revisited: the difference between two estimates. BMU 2003, 326:279.

13. Sackett DL. Why randomized controlled trials fail but needrit: 2. Failure to emplon physiological statistics, or the only formula a clinician-trialist is ever likely to need for understand!). CMAJ 2001;165:1226-37. 


\section{Chapter 9}

Radiography as primary outcome in rheumatoid arthritis: acceptable sample sizes for trials with 3 months follow-up

Bruynesteyn K, Landewé R, van der Linden Si, van der Heijde D

Ann Rheum Dis, accepted for publication 


\section{Abstract}

Aim

To investigate whether plain radiographs are able to reveal changes in joint damage due to rheumatoid arthritis (RA) within a 3 -month interval.

\section{Methods}

One-trundred-and-eighty-eight film pairs taken with a 3-month interval were evaluated. They were scored with (chronological) and without (paired) knowledge of the sequence of the fitms according to the Sharp/ran der Heijde method. Changes in joint damage were analysed on a group and on an individual level for different subsets of patients. Sample sizes required to detect statistically and clinically significant differences were estimated based on the percentages of patients with progression larger than the smallest detectable change (SDC).

\section{Results}

Changes in joint damage were seen by both the chronological and the paired scoring method. The percentage of patients with progression of joint damage larger than the corresponding SDCs (1.7 and 2.4) varied in the subsets from $18 \%$ to $64 \%$ if based on the chronologicall change-scores and from $9 \%$ to $36 \%$ using paired change-scores. Acceptable sample size estimates were seen in several subsets, depending 1 ) on how the investigated drug would reduce the individual risk on progression of joint damage (by an absolute or a relative risk reduction model), 2) on how damage was scored (chronological or paired), 3) on the baseline risk and 4) whether 2-sided or 1-sided tests would be used.

\section{Conclusion}

It is possible to reliably detect changes in point damage due to RA already within 3 months. This finding can be used to plan short-term randomised controlled trials with radiographic progression as primary outcome. 


\section{Introduction}

Prevention of structural damage is an important goal of rheumatoid arthritis (RA) therapy and recognized by the Food and Drug Administration (FDA) as a separate claim. 'Trials have to be at least one year in duration in order to label the drug as preventing structural damage. Recent trials have shown that differences in radiological progression between an experimental drug and a control group can be detected already after 6 months. ${ }^{2-4}$ Because phase II (dosefinding) trials are of sharter duration, often 3 months or less, structural damage has never been included as outcome measure in these trials. If it would be possible to detect progression of joint damage within 3 months of a phase-11 trial this would have clear advantages. The possible preventive effect of a drug on structural damage could then already be indicated in a very early stage. And even more, the optimal dose- and exposure range for slowing progression of radiological joint damage could then be defined. MRI and ultrasonography are sensitive assessments and are assumed to detect changes after 3 months. Plain radiographs are assumed to be too insensitive to detect changes in structural damage within an interval of 3 months, however this has never been investigated.

Primary aim of this study was to investigate whether the progression in 3 months can reliably be detected by measuring joint damage on plain radiographs.

\section{Patients and Methods}

\section{Methods}

Radiographs of a phase 11 multi-centre double blind randomised placebocontrolled trial were evaluated for this study. The trial investigated the efficacy and tolerability of a new compound in RA patients during 3 months. In this trial, superiority of the drug over placebo could not be demonstrated, both regarding disease activity parameters and radiographic joint damage. Therefore we considered all patients enrolled in this study as untreated controls.

\section{Patients}

Patients fulfilled the ACR criteria for RA. The patients enrolled were recruited from the RA population of both general and academic rheumatology centres. All patients had to have an active poly-arthritis with a Modified Disease Activity Score (DAS28) ${ }^{5}$ of 4.5 or more at screening. The subjects should not have failed treatment on more than 3 disease modifying anti-rheumatic drugs (DMARDs). Patients with a history of RA longer than 15 years or treated with a biological therapy during the last year were excluded from trial enrolment. Concomitant medication with stable dases of NSAIDs or oral corticosteroids (maximal $7.5 \mathrm{mg}$ 
prednisone or equivalent) was allowed during the trial periad. Intra-articular injections with corficosteroids were not allowed.

\section{Radiographic scoring method}

Posterior-anterior films of the hands and anterior-posterior films of the feet were made at baseline and at the end visit. The films were scored according to the Sharp/van der Heijde method ${ }^{6}$ by an experienced observer (KB) who was blinded for patient identity. The principal score used in analyses is the total score (max. 448), which is the sum of the erosion score and the joint space narrowing score. Radiographs have been scored in trials with and without knowledge of the chronological sequence of the films. So, it was decided to score the films according to both methods, with a reading interval of 2 months. For the chronological method, negative change-scores were allowed if the observer was convinced of repoir of the erosion(s).

\section{Statistical analyses}

Patients with hand and foot films at basieline as well as at the end visit, and with an interval of 3 months \pm 2 weeks were included in the analyses. Analyses were performed separately for early RA patients (RA duration less than 2 years since diagnosis) and late RA patients (RA duration of 2 years or more since diagnosis). Baseline characteristics with a Gaussian distribution were expressed as mean and SD; differences between the early RA and late RA patients were tested with independent t-tests. Non-Gaussian distributed baseline characteristics were expressed by medians and interquartile ranges (IQR) and tested with MannWhitney tests. Baseline characteristics with discrete distributions were expressed as counts (\%) and analyzed by continuity-corrected $\chi^{2}$ tests or Fisher's exact test when appropriate. The 3-months Sharp/van der Heilde change-scores were presented by the mean, standard deviation (SD), median, full range and interquartile ranges of change-scores $(I Q R)$. The percentage of patients with change larger than the smallest detectable change (SDC) was also determined. The SDC is a statistical concept representing the smallest difference between 2 succeeding scores of the same patient that can be interpreted as a "real" change beyond measurement error and was recommend as cutoff value in a consensus meeting on how to report radiographic data. The intra observer measurement error was used here to calculate the SDC, based on the $95 \%$ level of agreement. For this purpose, the observer read the films of 20 randomly selected patients again, 1 month after each reading session. The formulla to calculate this SDC is given in appendix 1. Differences in scores or percentages of patients with progression larger than the SDC between the chronological and paired method were analyzed with paired tests (paired tintest and the Wilcoxon test for the scores; the McNemar tests for the percentages].

To investigate whether the changes in joint damage observed in this study would be large enough to be useful as an outcome measure in future clinical trials of 3 months duration, sample sizes were estimated for imaginary trials. Sample sizes were estimated for several subsets of patients: early and late RA, as well as high(er) baseline risk for radiological progression of joint damage. The latter 
was done because randomised clinical trials often include patients with a high risk on the outcome of interest by selecting on baseline predictors for that outcome, this in order to achieve a high contrast between treatment groups. Baseline damage was chosen as baseline predictor for progression of damage. To check if this prognostic factor known from literature ${ }^{8-11}$ also operated as prognostic factor in this study we applied a logistic regression model, with correction for age, gender, RF-status and C-reactive protein level at baseline. This confirmed that baseline damage operated as an independent prognostic factor in our study too. The patients were split-up in 3 baseline risk groups by tertiles. The sample size estimates were based on the outcome variable: "percentage of patients with progression larger than the SDC" (i.e., on the progression of joint damage at the individual level). Sample size calculations based on mean group values were not performed due to skewness of radiological progression scores in RA. The sample sizes required were calculated for 3 types of drug mechanism: drugs (mainly) working according to a relative risk reduction (RRR) model, drugs (mainly) working according to an absolute risk reduction (ARR) model or drugs working according to a mix of both. We shall describe these models shortly; for a more in-depth discussion on the concepts of these models we refer to chapter 8 . According to the RRR model, the RRR (i.e., the reduction of the event rate in the treatment group in proportion to that in the placebo group) remains constant over the different baseline risk groups. As a consequence, the ARR (absolute reduction of negative event rate caused by experimental drug, i.e., the absolute difference in the event rates between placebo and the treatment group) varies with baseline risk of the patients. It a drug acts mainly according to the RRR model, selecting patients with a high baseline risk for the progression of joint damage will result in smaller sample sizes needed. In the ARR model, on the other hand, the ARR stays constant irrespective of baseline risk and the RRR varies over the different baseline risk groups. Selecting patients by baseline risk may have diverse effects on the sample size required and if a drug works according the ARR madel it is shown that it is wise to avoid patients with a baseline risk around 50\%. Note that risk reduction in the context of this article means the reduction of the number of patients with joint damage progression above the SDC. For the models 2 hypothetical treatment effects were evaluated. For the RRR model: a constant RRR of $50 \%$ and $75 \%$. For the ARR model: a constant ARR of $15 \%$ and $25 \%$. Finally, we also determined the sample sizes required for a situation in which $5 \%$ of the patients in the experimental treatment group do not respond, irrespective of baseline risk. This results in mix of both models: increasing ARR and increasing RRR with higher baseline risk. These hypothetical risk reductions are in our opinion all clinically relevant treatment effect. Descriptive analyses, statistical testing and the logistic regression model were performed with SPSS, version 10.0 for Windows (Chicago, IL). All sample size calculations were performed with the power calculator from the UCLA department of statistics with the 2sample arcsine approximation of the binomial distribution with $\beta$ set at 0.20 and $\alpha$ at 0.05 (http://calculators.stat.ucla.edu/ powercalc/). Sample size calculations were performed for two-sided statistical testing as well as for one-sided statistical testing. 


\section{Results}

Two hundred and thirty-five patients had films at baseline and end of the study. Of these, 188 patients, i.e., $80 \%$ had a correct interval of 3 months (range 2.5. $3.5)$ and were included in this study. The mean follow-up of the included patients was 91 days (SD 4.0) in the early RA group and 92 days (SD 4.5) in the late RA group. The baseline characteristics of the 188 patients included are shown in Table 9.1. The mean baseline damage scores of all patients, including those with a follow-up of less than 2.5 or more than 3.5 months, were comparable with those of the included patients (data not shown).

\section{TABLE 9.1}

Baseline characteristics of the patients

\begin{tabular}{|c|c|c|c|c|}
\hline Variable & $\begin{array}{l}\text { Al patients } \\
(n=188)\end{array}$ & $\begin{array}{l}\text { Eorly RA } \\
(n=96\end{array}$ & $\begin{array}{l}\text { Late RA } \\
n=92)\end{array}$ & $\begin{array}{c}\text { Difference } \\
\text { late RA-early RA }\end{array}$ \\
\hline & & & 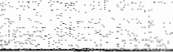 & P-value \\
\hline RA duration since & 4.5 & 1.2 & 8.5 & $<0.0001$ \\
\hline onsel syrnptoms (yrs)" & $(1.1-9.1)$ & $(0.5-2.7)$ & $(5.6-11.9)$ & \\
\hline RA duration since & 1.8 & 0.2 & 6.9 & $<0.0001$ \\
\hline diagnosis (yrs) & $(0.2-6.7)$ & $(0.05-0.7)$ & $(4.2-9.6)$ & \\
\hline \multirow[t]{2}{*}{ Age $(y r s)^{\dagger}$} & 56.3 & 57.3 & 55.2 & 0.24 \\
\hline & $(12.5)$ & $(12.8)$ & $(12.0)$ & \\
\hline \multirow[t]{2}{*}{ DAS28 } & 5.7 & 5.8 & 5.7 & 0.51 \\
\hline & $(1.1)$ & $(1.0)$ & $(1.2)$ & \\
\hline Serum C-reactive & 1.5 & 1.1 & 1.8 & 0.04 \\
\hline protein (mg/dll) & $(0.8-3.5)$ & $(0.7-3.3)$ & $(1.0-3.5)$ & \\
\hline Health Assessment & 1.1 & 1.0 & 1.2 & 0.04 \\
\hline Questionnaire score $(0-3)^{\dagger}$ & $(0.7)$ & $(0.7)$ & $(1.1)$ & \\
\hline Positive serum test for & $67 \%$ & $59 \%$ & $75 \%$ & 0.03 \\
\hline \multicolumn{5}{|l|}{ theumaloid arthritis $(\%)$} \\
\hline DMARD naive $(\%)^{*}$ & $38 \%$ & $69 \%$ & $7 \%$ & $<0.0001$ \\
\hline Corticosteroids use $(\%)^{i, n}$ & $27 \%$ & $24 \%$ & $30 \%$ & 0.40 \\
\hline Female $(\%)^{*}$ & $68 \%$ & $64 \%$ & $72 \%$ & 0.30 \\
\hline Chronological damage & 7.5 & 3.0 & 21.5 & $<0.0001$ \\
\hline score $(0.448)^{2}$ & $(1-33)$ & $(0-13)$ & $(5-56)$ & \\
\hline Paired damage score & 9.0 & 3.0 & 23.5 & $<0.0001$ \\
\hline$(0.448)^{\circ}$ & $(2-33)$ & $(0-12)$ & $(6-58)$ & \\
\hline
\end{tabular}

Early RA disease duration 2 years, Late RA. disease duration 22 years * median Ilnter Quartile Range) difference between early and late PA pritenls tesied with Mann whithey tests 1 mean (SD); difference between early and late RA patients tested with independent t tests, 1 difference between early and late RA patients tested with chi square tests, max $75 \mathrm{mg}$ prednisone or equivalent 
Table 9.2 shows the 3 -month changes in radiological joint damage at a group level. Both the chronological scoring method and the paired scoring method picked up progression of radiological joint damage within the 3-month interval. For the chronological method, all change-scores were $\geq 0$, so no elear repair of erosions was observed in the 3 months when the observer knew the chronological sequence of the films. The changes scored with known chronology were (statistically) higher than the changes scored without knowledge of the chronological sequence; both the paired t-test and the Wilcoxon resulted in pvalues of 0.03 and $<0.0001$ in the early and late RA group, respectively. Progression of joint damage was the largest in the late RA group. The difference in change-scores between the early and late RA groups was statistically significant for the change-scores if scored with the chronological method (independent $t$-test $P=0.001$ and Mann-Whitney $P=0.002$ ) but not if scored without information on the chronological order (independent t-test $P=0.15$ and Mann-Whitney $\mathrm{P}=0.18$ ).

\section{TABLE 9.2}

Group changes in joint damage within 3 months; measured with (chronological) and without (paired) knowledge of the chronological order of the films.

\begin{tabular}{|c|c|c|c|c|}
\hline \multirow[b]{2}{*}{ Palient giroup } & \multicolumn{2}{|c|}{$\begin{array}{l}\text { Chronological Sharp/van der } \\
\text { Heilde change-scores }\end{array}$} & \multicolumn{2}{|c|}{$\begin{array}{l}\text { Paired Sharp/van dei Heijde } \\
\text { Change-scales }\end{array}$} \\
\hline & mean (sd) & median frange/IIOR] & $\operatorname{mean}(\mathrm{sal})$ & median frange) [QR] \\
\hline Eorly RA $(n=96)$ & $0.8(1.7)$ & $0.0(0-8) ;[0-1]$ & $0.5(1.5)$ & $0.0(-4 ; 6) ;[0 ; 1]$ \\
\hline Late RA $(n=92)$ & $2.0(3.0)$ & $1.0(0-13):[1-4]$ & $1.0(2.7)$ & $0.0(-10 ; 12) ;[0 ; 2]$ \\
\hline
\end{tabular}

Early $R A$. disease duration $<2$ years, Late $R A$. diseaise duration $\geq 2$ years, lQR. Interquiartile range

Table 9.3 shows the percentage of patients with progression of joint damage larger than the SDC at 3 months. The SDC was 1.7 for the chronological scoring method and 2.4 for the paired scoring method. The differences in the number of patients with progression above the SDC between the chronological and the paired method were statistically significant in both the early RA group and the late RA group $(P=0.04$ and $P<0.001$, McNemar test). The late RA group contained more patients with progression larger than the SDC. This difference was statistically significant if damage was scored with the chronological method $\left(\mathrm{P}=0.008, \chi^{2}\right.$ test with continuity correction), but was not when using the paired scoring method $\left(P=0.16, \chi^{2}\right.$ test with continuity correction). If patients with a high(er) risk for progression were selected, using the baseline damage scores as prognastic factor, the percentage of patients with progression of joint damage above the SDC increased considerably. The increase in percentage of patients with progression above the SDC with increasing baseline risk was statistically significant for both the chronological 
and the paired method, in the early $R A$ group as well as the late RA group ( $p$ values $<0.0001-0.001, x^{2}$ tests for trend).

TABLE 9.3

Changes in joint damage within 3 months, expressed on the individual level for different risk groups based on the baseline jaint damage; measured with (chronological) and without (paired) knowledge of the chronological order of the films.

\begin{tabular}{|c|c|c|}
\hline Pafient group & $\begin{array}{l}\text { Percentage of patients with } \\
\text { progression }=5 \mathrm{SQC} \text {, based } \\
\text { on the chronological } \\
\text { change-scores }\end{array}$ & $\begin{array}{c}\text { Percentage of patients } \\
\text { wh progression } \geq \text { SOC } \\
\text { based on the pained } \\
\text { change-scores }\end{array}$ \\
\hline Early RA $(N=96)$ & $\%(\mathrm{n} / \mathbb{N})$ & $\%(n / N)$ \\
\hline Regardless of baseline damage & $18 \%(17 / 96)$ & $9 \%(9 / 96)$ \\
\hline $\begin{array}{l}\text { Low baseline damage, } \\
\text { scone } \leq 3\end{array}$ & $2 \%(1 / 50)$ & $0 \%(0 / 52)$ \\
\hline $\begin{array}{l}\text { Medium baseline damage, } \\
\text { score } 4-20\end{array}$ & $22 \%(7 / 32)$ & $13 \%(4 / 30)$ \\
\hline $\begin{array}{l}\text { High baselline damage, } \\
\text { score } \geq 21\end{array}$ & $64 \%(9 / 14)$ & $36 \%(5 / 14)$ \\
\hline \multicolumn{3}{|l|}{ Late RA $N=92)$} \\
\hline Regardless of baseline damage & $36 \%(33 / 92)$ & $17 \%(16 / 92)$ \\
\hline $\begin{array}{l}\text { Low baseline damage, } \\
\text { score } \leq 3\end{array}$ & $6 \%(1 / 16)$ & $6 \%(1 / 16)$ \\
\hline $\begin{array}{l}\text { Medium baseline damage, } \\
\text { score } 4-20\end{array}$ & $25 \%(7 / 28)$ & $11 \%(3 / 28)$ \\
\hline $\begin{array}{l}\text { High baseline damage, } \\
\text { score } \geq 21\end{array}$ & $52 \%(25 / 48)$ & $25 \%(12 / 48)$ \\
\hline
\end{tabular}

Eorly RA: disimase duration $<2$ years, Late RA. disease duration $\geq 2$ years; $S D C$ : smallest détedable change (see dse rabhod section, SDC chronological 12,1 SDC paired 2.4

Table 9.4 and 9.5 present the sample sizes required to detect statistically and clinically significant differences in percentages of patients with progression larger than the SDC between an imaginary experimental intervention group and a placebo group, based on the chronological (Table 9.4) and paired (Table 9.5) placebo event rates found in this study. Note that the sample sizes are calculated with the un-rounded percentages, so sample sizes for the early RA group with a paired baseline damage score of 4 or higher $(20,5 \%, 9 / 44)$ differ from those from the late RA group with a paired baseline damage score of 4 or higher $(19,7 \%, 15 / 76)$ 
Radiographic joint damege progression in 3 months

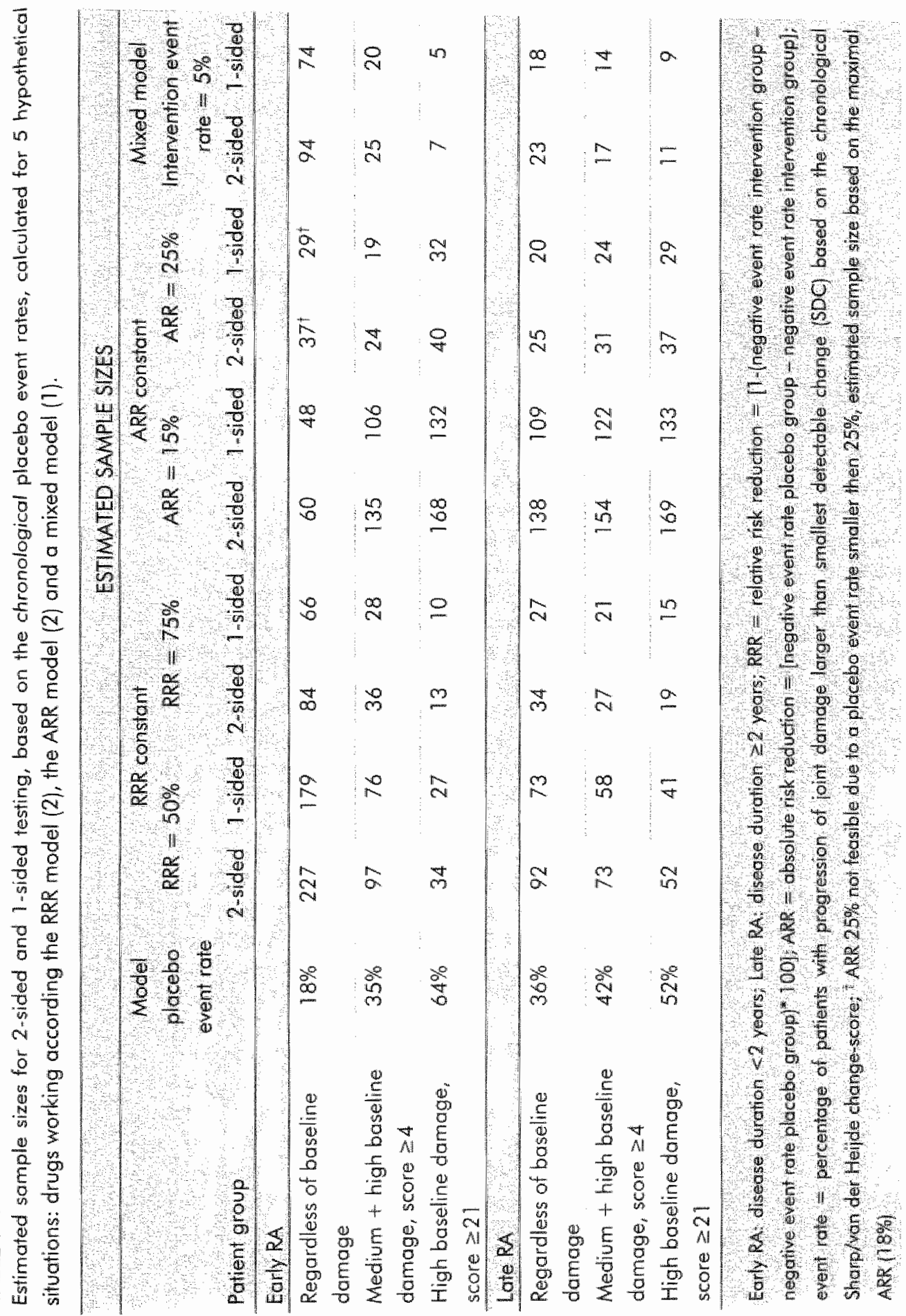




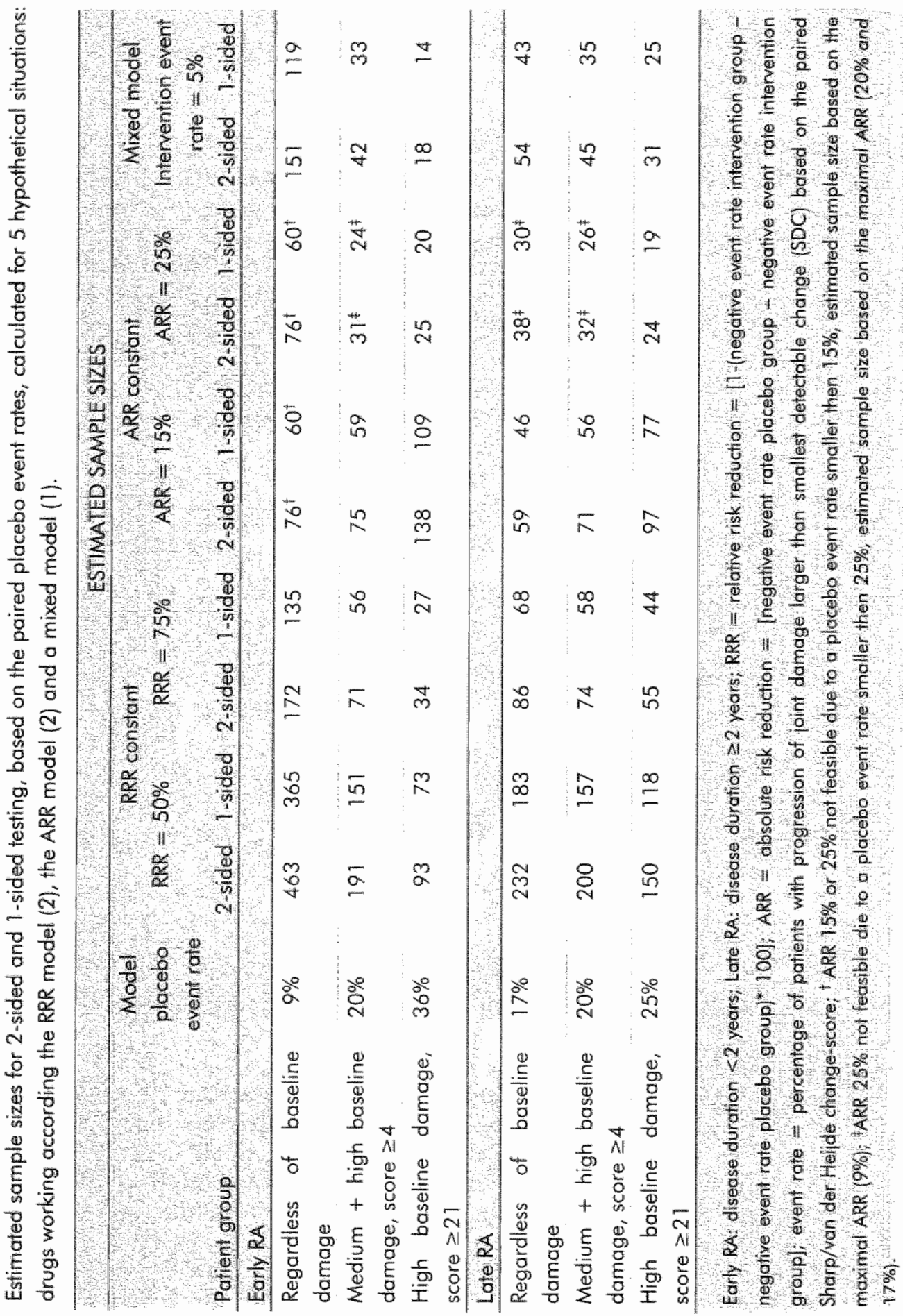


One can see that, for example, if scoring with knowledge of the chronological sequence of the films, 227 patients per group would be required to demonstrate a RRR of $50 \%$ in the early RA group. A RRR of $50 \%$ in this situation means a reduction of the negative event rate in the intervention group $109 \%(0.50 * 18 \%)$.

\section{Discussion}

This study showed that even within a 3.month interval changes in joint domage in patients with active RA could be detected on plain radiographs. Although the majority of patients showed no progression in these 3 months - the data were highly skewed - a substantial part of the patients showed unequivocal progression of joint damage. For example, $18 \%$ of the early RA patients and $36 \%$ of the late RA patients had change-scores above the SDC if scored with known chronology.

Whether progression of joint damage at the level as found in this study would provide a sufficient large contrast between treatment groups of a phase II placebo controlled dimical trial to defect dinically and statistically significant differences depends on the overall power of the trial. The power of a study depends 1) on the contrast between the groups under study, 2) on the samples size of the groups, 3) on the risk level accepted for rejecting the null hypothesis that the treatment effects are equal when this null hypothesis is in fact true (type 1 error, "false-positive" result), and 4) whether 1-sided or 2-sided confidence intervals or p-values are appropriate. The contrast between groups under study depends, besides the actual treatment effect, also on a) the sensitivity of measurement instrument (e.g., radiological scoring method) used to detect the changes and on b) the mechanism of the risk reduction.

So, in order to statistically underscore a clinically significant difference between treatment groups, the treatment effect of the drug under study achieved in a 3 month interval first needs to be large enough. Studies on 2 of the recently approved anti-rheumatic drugs, lefiunomide and infliximab's, showed both a RRR around the $80 \%$ and an ARR of $14 \%$ by leflunomide and $25 \%$ by infliximab. Leflunomide reduced the percentage of patients with an erosion change-score larger than 3 units in 6 month from $17 \%$ in the placebo group to $3 \%$ in the leftunomide group. Infliximab showed a reduction of patients with progression larger the accompanying SDC in one year from $37 \%$ in the MTX group to $6 \%$ in the groups in which infliximab was added. The hypothetical RRR and ARR used in Table 9.4 and 9.5 to estimate the sample sizes are therefore not only dinically relevant but represent also realistic trearment effects.

Second, the number of patients included also determines the statistical power of trial. The number of patients per group that is acceptable for a phase II trial is usually around 60 per group. Table 9.4 and 9.5 show that for several patient scenarios 60 patients or less per group is sufficient. If 60 potients is sufficient depends - as mentioned-on 1) an how the drug under investigation will reduce the individual risk on the progression of joint damage (by an absolute or a 
relative risk reduction model, 2) which instrument is used to score the damage (with or without knowledge of the sequence of the films) and 3) whether 2-sided or 1-sided tests will be used. If, for example, a drugs works according to the relative risk reduction model, the damage is scared chronologically, and 2-sided tests are used: a RRR of $75 \%$ can be determined statistically significant in early RA patients with intermediate and high baseline damage and all late RA patients irrespective of baseline damage. A RRR of $50 \%$ will show statistically significant differences in patients with high baseline damage but not in the other patient groups. If joint damage is scored paired instead of chronologically, only a RRR of $75 \%$ in a high baseline damage group will detect a statistically significant difference. Research on the risk reduction model of treatments outside the field of rheumatology showed constant RRR with varying ARR for the vast majority of the treatments. How disease madifying antirheumatic drugs (DMARD) reduce the individual risk on progression of joint damage has, to our knowledge, not been investigated before. In the accompanying short paper, we used the data of 2 recent trials to investigate the risk reduction on progression of joint damage due to RA in these trials. Future research shall have to reveal whether other DMARDs show equal patterns of risk reduction. More knowledge on how (groups of) DMARDs reduce the risk on progression of joint damage will make it possible to further optimize the designs of studies. For phase II trialls, the knowledge of the potential mechanism of action of the drug gained out of the multiple pre-clinical model systems can direct the choice for type of risk reduction model used for the sample size calculations. The differences in sample size between the estimates based on the placebo event rates if scored in chronological sequence or scored in pairs without information on the sequence is because the chronological method picked up more change in joint damage. It is known from literature that knowledge of the chronological sequence of films leads to higher proportions of patients with progression of joint damage than random reading. ${ }^{13.15}$ It is argued that knowledge of the chronological order provides the reader a maximum of information, thereby reducing the measurement error caused by variation of positioning of the hands and feet or variation in film quality. Results of a recent study ${ }^{16}$ even suggested that knowledge of the chronological sequence leads to an increase in detection of clinically relevant changes without serious overestimation of non-relevant changes. Consequently, if a drug would work according to a RRR model, the estimated sample sizes were lower if scored by the chronological method than by the paired method. However, for a drug working according to the absolute model the opposite was found: the estimated sample sizes were lower for the damage scores by the paired method. This is explained by the fact that the placebo event rates based on the chronological method approximated the 50\% progression rate more closely than those based on the paired method. One exception can be seen in table 5: in the hypothetical situations that a drug could achieve an ARR of $25 \%$, the sample size estimates for the early RA patients, irrespective of baseline damage, are lower if based on the chronological placebo event rates compared to the placebo events rates if scored with the paired method. The explanation for this finding is that both scoring methods show placebo event rates much smaller than $25 \%$. Only an 
ARR of $9 \%$ for the paired method and $18 \%$ for the chronological is possible in such a patient group and a reduction of $18 \%$ to $0 \%$ show statistically significant results with fewer patients than the reduction of $9 \%$ to $0 \%$. In the situation that a drug works according a more mixed model, the chronological scoring method again would be more favorable in all settings.

While the ethical debate on rheumatological phase II trials often focuses on the use of placebo controls, estimations of the required sample size are usually not evaluated to its ethical implications. Table 9.4 and 9.5 showed that in many settings it matters considerably whether a 2 -sided test or a 1 -sided test is chosen as the base of sample size estimations. One-sided testing is mostly considered unacceptable because it does not account for the possibility that the reference group might be better. Knottnerus and Bouter ${ }^{17}$ recently revitalized the discussion by stating that 1 -sided testing and corresponding sample size estimations can be proposed as the preferred approach if 1) the scientific hypothesis to be tested is obviously 1 -sided or if 2) only a clear advantage in effect of the principal over the reference interventions should have consequences for practice. They thus argued that in placebo controlled clinical trials 1 -sided testing would be adequate and even the default option. We agree with this view, especially for radiographic progression, and are therefore in favor of the 1-sided sample sizes. To give a complete overview, we presented the estimated sample sizes based both on 2 -sided and on 1 -sided testing.

In this study the RA patients with disease duration of 2 years or more, which were all active at inclusion (mean DAS 5.7), showed overall more progression of joint damage than the early RA group. A possible explanation for this can be that a large part of the late RA patients already have proven to be aggressive and treatment resistant (active polyartritis and substantial radiological joint damage despite DMARD use). The early RA patients that had proven to have a worse prognosis by already showing a joint damage at baseline of 21 or more, on the other hand, deteriorated more compared to the late RA patients with similar baseline damage. Further, the differences in baseline characteristics found between the early RA and the late RA group were understandable from the pathophysiological mechanism of joint damage by RA.

It is known that radiological progression scores show a highly skewed distribution pattern. In studies over a period of 6 to 12 months, the majority of patients showed no- or only mild progression and only subsets of patients showed substantial progression. ${ }^{18}$ Such skewed distributions require mathematical transformation for appropriate parametric statistical testing, nonparametric statistics or the data should be analyzed in a dichotomized fashion as was done in this study. Analyses on an individual dichotomized level of a continuous outcome measure are considered less sensitive to detect differences between treatment groups. Question is whether this is also true for data that are skewed like radiological progression in a 3-month interval. The sample size estimations in this study anyhow showed acceptable numbers of patients with which clinically important dichotomous treatment effects can be detected.

In summary, it is possible to detect changes in joint damage due to RA already within 3 months with plain radiographs. This study further showed that whether 
this change in joint damage will be large enough to statistically underscore clinically relevant treatment differences in a placebo controlled clinical trial depends on 1) how the drug under investigation will reduce the individual risk on the progression of joint damage (by an absolute or a relative risk reduction model), 2) on how the damage will be scored (with or without knowledge of the sequence of the films), 3) the baseline risk of the patients investigated and 4) whether 2 -sided or 7 -sided tests will be used. We conclude that it is feasible to get an impression whether or not an investigational drug is capable of retarding radiographic progression in placebo controlled trials with only 3 months followup.

\section{Appendix}

Formula of the $95 \%$ SDC based on an analyses of variance with 2 factors; the patient's change-score $(\mathrm{p}, 188$ levels $)$ and the observer $(0,2$ levels).

$\pm 1.96 * \sqrt{\sigma^{2}(o)+\sigma^{2}(p * a, e)}$ 


\section{References}

1. Guidance for Industry. Clinical development for drugs, devices and biological products for the treament of Rheumatoid Arthritis. (RA). Rockville: Center for Drug Evaluation and Research, Food and Drug administration, 1997:1-56.

2. Boers $M$, Verhoeven $A C$, Markusse HM, van de Laar $M$, Westhovens $R$, van

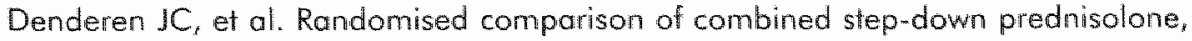
methotrexate and sulphasalazine with sulphasalazine alone in early theumatoid arthritis. Lancet 1997;350:309-18

3. Bathon JM, Martin RW, Fleischmann RM, Tesser IR, Schiff MH, Keystone EC, Genowese MC, Wasko MC, Moreland LW, Weaver AL, Markenson J, Finck BK. A comparison of etanercept and methotrexate in patients with early rheumatoid arthritis. N Engl J Med 2000;343:1586-93.

4. Sharp IT, Strand $V$, Leung $H_{4}$ Hurley $F$, Loew-Friedrich I. Treatment with leflunomide slows radiographic progression of theumaloid arthritis: results from three randomized controlled trials of leflunomide in patients with active theumatoid arthritis. Leflunomide Rheumatoid Arthritis Investigators Group. Arthritis Rheum 2000;43:495-505.

5. Prevoo ML, van " Hof $M A$, Kuper $H H$, van Leeuwen $M A$, wan de Putte $L B$, van Riel PL. Modified disease activity scores that include twenty-eight-joint counts. Development and validation in a prospective longitudinal study of patients with rheumatoid arthritis. Arthritis Rheum 1995;38:44-8.

6. van der Heijde D. How to read radiagraphs according to the Sharp/van der Heijde method. I Rheumatol 2000;27:261-31.

7. van der Heijde D, Simon L, Smalen J, Strand $V$, Sharp J, Baers M, Breedveld F, Weisman $M$, Weinblatt $M$, Rau $\mathbb{R}_{*}$ Lipsky $P$. How to report radiographic data in randomized clinical trials in rheumatoid arthritis: guidelines from a roundtable discussion. Arthritis Rheum 2002;47:215-8.

8. Uhlig T, Smedstad LM, Vaglum P, Moum T, Gerard N, Kvien TK. The course of rheumatoid arthritis and predictors of psychological, physical and radiographic outcome after 5 years of fallow-up. Rheumatology (Oxford) 2000,39:732-41.

9. Boers $M$, Kastense PJ, Verhoeven AC, van der Linden S. Inflammation and damage in an individual joint predict further domage in that joint in patients with early rheumatoid arthritis. Arthrisis Rheum 2001;44:2242-6.

10. Combe B, Dougados M, Goupille P, Cantagrel A, Elicou JF, Sibilia d, Meyer O, Sany J. Daures IP. Dubois A. Prognostic factors for radiographic damage in early rheumatoid arthritis: a multiparameter prospective study. Arthritis Rheum $2001 ; 44: 1736-43$.

11. Drossaers-Bakker KW, Zwinderman AH, Vlieland TP, Van Zeben D, Vas K, Breedveld FC, et al. Long-term outcome in rheumatoid anthritis: a simple algarithm of baseline parameters can predict radiographic damage, disability, and disease course at 12 . year followup. Arthritis Rheum $2002 ; 47: 383-90$. 
12. Lipsky PE, van der Heijde DM, St Clair EW, Furst DE, Breedveld FC, Kalden JR, Smolen JS, Weisman M, Emery P. Feldmann M, Harriman GR, Maini RN; AntiTumor Necrosis Factor Trial in Rheumataid Arthritis with Concomitant Therapy Study Group. Infliximab and methatrexate in the treatment of rheumatoid arthrifis. Ant:Tumor Necrosis Factor Trial in Rheumatoid Anthritis with Concomitant Therapy Study Group. N Engl J Med 2000;343:1594-1602.

13. Ferrara R, Priolo F, Cammisa M, Bacarini L, Cerase A, Pasera G, Ferraccioli GF. Alberighi $O D$, Antonellini A, Marubini E. Clinical Trials In Rheumatoid Arthritis: Methodological Suggestions For Assessing Radiographs Arising From the Grisar Study. Anm Rheum Dis 1997;56:608-12.

14. Salaffi F, Carolti M. Interobserver Variation in Quantitative Analysis Of Hand Radiographs In Rheumatoid Arthritis: Comparison of 3 Different Reading Procedures. J Rheumatol 1997;24 (10):2055-6.

15. van der Heijde D, Boonen $A$, Boers $M$, Kostense $P$, van der Linden 5 . Reading radiographs in chronological order, in pairs or as single films has important implications for the discriminative power of rheumatoid arthritis clinical trials. Rheumatology (Oxford) 1999;38:1213-20.

16. Bruynesteyn $K$, wan der Heijde $D$, Boers $M$, Saudan $A$, Peloso $P$, Paulus $H$, Houben $H$, Griffiths B, Edmonds J, Bresnihan B, Boonen A, Van Der Linden S. Detecting radiological changes in theumatoid arthritis that are considered important by clinical experts: influence of reading with or without known sequence. I Rheumatol $2002 ; 29: 2306 \cdot 12$.

17. Knotmerus JA, Bouter LM. The ethics of sample size: two-sided festing and one-sided thinking. J Clin Epidemiol 2001;54:109-10.

18. Landewe $R$, Boers M, Van Der Heijde D. How to interpret radiological progression in randomized dinical trials? Rheumatology (Oxford) 2003:42:2 2 . 


\section{Chapter 10}

\section{General discussion and conclusions}

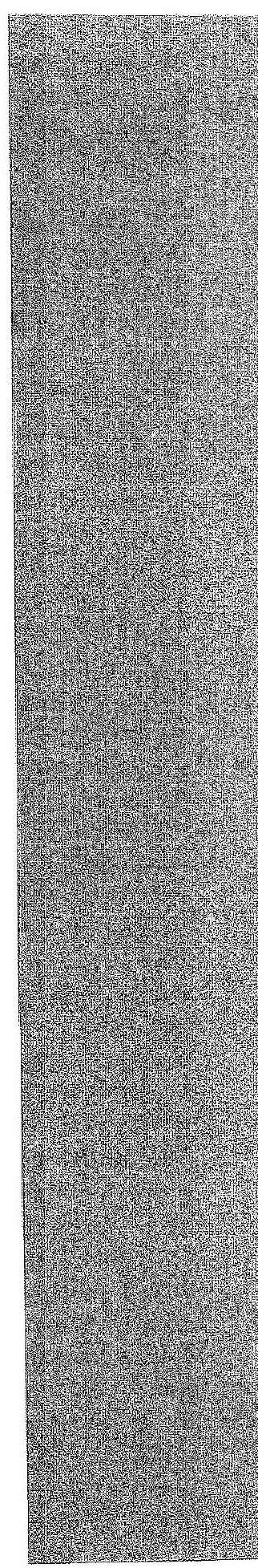




\section{General discussion and conclusions}

In chapters 2-9 several different methodological issues are addressed regarding the Sharp/van der Heijde and Larsen/Scott radiological scoring methods. In this chapter the results and conclusions of these chapters are summarized and the implications and recommendations for future research discussed.

\section{Reliability of the Sharp/van der Heijde and Larsen/Scott scoring method}

In chapter 2, the reliability of the Sharp/van der Heiide and the Larsen/Scatt method - expressed as the discriminative capacity and responsiveness - were compared by statistics based on the generalizability theory. The analyses confirmed the discriminative capacity and responsiveness of the 2 methods ${ }^{1.4}$. Moreover they gave the opportunity to investigate how the reliability of both methods related to each other. It could be concluded that the discriminative capacily and responsiveness were highly acceptable and comparable when comparing mean group scores. However, when comparing individual patients, the Sharp/van der Heijde status scores were measured with greater precision and the method was also more sensitive in detecting change over 1 year. For standard group analyses on radiological joint damage in trials or cohort studies it should thus not matter whether one uses the Sharp/van der Heijde method or the Larsen/Scott method. However, if one is interested in how many patients the radiological joint damage actually deteriorated or in how many patients repair was seen, the Sharp/ van der Heiide method thus seems preferable. Recent guidelines on how to report radiographic data in RCTs in RA has advocated to also include the percentage of patients that are (non) responders ${ }^{5}$ This percentage of (non) responders is especially interesting information for practising rheumatologist: which helps him/her to individualize group data from clinical research, the ultimate goal of evidence-based medicine (EBM) and it enables the health care workers to calculate the number needed to treat (NNT). ${ }^{6,7}$

The study results of chapter 2 further once more showed that the sole use of intra-class correlations coefficient could give misleading information. In general, when testing the reliability of a new outcome measurement, statistics like the standard error of measurement (SEM), smallest detectable difference (SDD) or smallest detectable change (SDC) - statistics that all express the actual measurement error - should also be included.

Finally, it should be noted that the reliability of a scoring method is not context free. ${ }^{B Q}$ Reliability - discriminative capacity and sensitivity to change-can differ for different patient groups but also for different time intervals. In the study of chapter 2, the time interval between films was 1 year, corresponding to the interval required by the American Food \& Drug Association (FDA) for a drug claim of prevention of radiological joint damage due to $R A{ }^{10}$ Recent trials, however, have shown that differences in radiological progression between an 
experimental drug and a control group can already be detected after 6 months. ${ }^{11-13}$ Furthermore, the study results presented in chapter 9 revealed that it is possible to reliably detect changes in joint damage due to RA already within 3 months. In the future, the inferval between films to assess an effect on structural damage might be shortened. Further, the reliability statistics for the films with a 3 -month interval estimated in chapter 9 were very good and suggest that the sensitivity to change of the Sharp/ van der Heijde method for films is better for films with a shorter interval than 1 year. Future studies will have to reveal whether the found differences in sensitivity lo change between the Sharp van der Heijde and Larsen/Scott scoring methods are also true when used to defect changes with shorter intervals than 1 year and how the sensitivity statistics for films scored with different time intervals relate to each other in general.

\section{Optimal number of readers}

Chapter 2 also addressed the issue of the optimal number of readers to be used when scoring films. It was shown that the discriminatory and evaluative capacity of the scoring methods on a group level did not change substantially by adding 1 or 2 readers, given that the readers had the same training and experience. The reliability of the individual scores, on the other hand, did increase substantially by adding readers. The greatest gain was reached by the increase from 1 to 2 readers. Similar results have been found by Fries et al. ${ }^{14}$ They concluded that the use of more than 2 readers introduces extra costs with (relatively) little gain. All in all, it may be concluded that from a generalizability point of view, the optimal number is 2 readers when scoring radiological joint damage due to RA with the Sharp/van der Heijde or Larsen/Scott method.

\section{Analyses on non-eroded joints}

In the context of a trial, prevention of erosions in undamaged joints is often considered more important than prevention of progression in already damaged joints. Chapter 3 showed that important information and discriminative strength might be lost when assessment is limited to the development in undamaged joints. It was consequently concluded that in trials information on the number and weight of erosions should be assessed regardless the previous state of the joints, especially because at that time trials had moderate power to detect difference in progression. The fact that recent studies suggest that reparative changes in erosions also seem possible on group levell ${ }^{15}$, makes it even more important to evaluate joint damage in already eroded jaints.

Expert-based Minimal Clinically Important Difference (MCID) of the Sharp/van der Heijde and Larsen/Scott methods

To improve interpretation of clinical trial results these are offen also expressed as the proportion of patients in which actually a (clinically relevant) change in outcome measure was seen, as mentioned above. Chapter 4 described the definition of the so-called Minimal Clinically Important Difference (MCID) of the 
Sharp/ van der Heijde and the Larsen/Scott method by an international expent panel of rheumatologists for four clinical settings (early versus advanced RA and mild versus high disease activity). In the past it has been advocated to use the SDD as minimum cutoff level for reporting patients with and without radiographic progression of joint damage, so it was also investigated how the expert-based MCID related to the corresponding SDD. ${ }^{16}$ In this study the expert panel judged changes in joint damage around the level of the SDD of the Sharp/van der Heijde method as minimally clinically important. However the expert-based MCID expressed in Larsen/Scott units was much smaller than the Larsen/Scott SDD. If one uses the SDD of the Larsen/Scott method as cutoff value, one should be aware that one might categorize joint damage of a considerable number of patients as stable while a panel of experts would have defined the change in joint damage patient as clinically important. And the other way around, the expert based MCID cannot be distinguished from measurement error.

Due to progressive understanding of the methods to assess measurement error, it became clear that in the situation that successive films of a particular patient are read side by side, i.e., simultaneously, the SDD overestimates the measurement error and is inappropriate to use as cutoff level for reporting patients with and without radiographic change. Chapter 7 described why the SDD overestimates the measurement error in this particular setting and presented how to estimate the more appropriate smallest detectable change (SDC). The SDD is still the measure to assess if the change in a particular patient is different from the change in another patient, while the SDC is the appropriate measure to determine if change is present in an individual patient.

Due to these new insights it was thus learnt that the SDC should have been used instead of the SDD in the study described in chapter 4 the films of the patients of this study were also read side-by-side). Calculation of the SDC of the Sharp/van der Heiide method for this study results in a SDC of 3.5 units. The SDC thus furns out to be around the MCID level for early RA patients with high disease activity (MCID 3.0) and to be somewhat smaller than the MCID in the settings "early RA with mild disease activity" and "advanced RA with high disease activity" (MCID 4.5). Compared to the MCID for advanced RA patients with mild disease activity the SDC however is considerably smaller (MCID 6.5). If the SDC of the Sharp/van der Heijde would be used as cutoff value to detect clinically relevant progression as defined by the expert panel, this would result in a sensitivity of 100\% and a specificity of $52 \%$ in the setting with "advanced RA patients with mild disease activity", but for the other settings this results an average sensitivity of $85 \%$ and specificity of $70 \%$.

The SDC of the Larsen/Scott method in the study of chapter 4 is estimated at 4.1 units. This is still much larger than the MCID for three clinical settings, except for the setting with patients with advanced RA and a high disease activity (MCID 4.5). If the SDC of the Sharp/van der Heijde would be used as cutoff value to detect clinically relevant progression as defined by the panel, this would result in a sensitivity of $80 \%$ and specificity of $92 \%$ for advanced RA patients with mild disease activity. But for the other 3 settings this results in an average sensitivity of 
$41 \%$ and specificity of $100 \%$. Thus, although the SDC of the Larsen/Scott method is smaller than the SDD the results above show that in many settings also the Larsen/Scott SDC seems to be too insensitive to use as cutoff value for individual dinically relevant change.

One part of the evaluation of the usefulness of expert panels like used in chapter 4 is to assess the consistency of the panel's judgments. Another aspect that should be investigated is the validity of the panel, which is ideally done by comparing the results with a so-called gold standard. Without the availability of a gol standard, the scores can alternatively be compared with the judgments of a panel composed of panellists of (an) other discipline(s). In chapter 5 the judgments of the panel of rheumatologists used for the study in chapter 4 were compared with the judgments of a couple of experienced musculoskeletal radiologists. The results showed that the panel of rheumatologists were inclined to change therapy in cases not reported as substantially progressive by the radiologists. The radiologists also judged less films as progressive than the rheumatologists. This study thus once more showed that the type of profession could strongly influence panel judgments. From a generalizability point of view it therefore seems important - if possible - to include more than one profession in an expert panel.

\section{Reading films with or without knowledge of the sequence of the films}

Former research has shown that reading with known sequence of films (chronological reading) enhances the sensitivity to change of a scoring method compared to reading films grouped per patient but presented without knowledge of the sequence (paired reading). ${ }^{2}$ It could however not be excluded that this was primarily caused by expectation bias rather than the diminishment of measurement error. In other words, the question remained whether the extra signal picked up by the readings in chronological order was principally bias or indeed a more precise signal. In chapter 6 a study is described that determined the influence of the reading order on the ability of the Sharp/van der Heilde and Larsen/Scott scoring method to detect clinically relevant progression of radialogical damage in the individual early $R A$ patient with high disease activity defined by an expert panel as external criterion. This study suggested that knowledge of the chronological sequence leads to an increase in detecting clinically relevant changes in the patient without seriously overestimating nonrelevant differences. Scoring without knowledge of the sequence of the films became less reproducible, resulting in increasing measurement error and thus larger SDD.

In the study described in chapter 6, again the SDD was used instead of the SDC (chapter 7). If the SDC had been used as threshold, the sensitivity to detect clinically relevant progression would increase significantly from $33 \%$ to $73 \%$ for the Sharp/van der Heijde method when the readers were informed about the sequence of the films. The specificity however then would diminish from $100 \%$ to $69 \%$. These new results thus suggest that knowledge of the chronological sequence indeed leads to a substantial increase in detecting clinically relevant 
changes but at the costs of some overestimation of progression regarded as non-relevont by the clinicions.

Taking all things above in consideration, should one conclude in recommending chromological reading instead of paired reading? The answer to this question is not straightforward. As discussed in chapter 6, knowledge of the sequence of films can introduce bias due to the fact that readers mostly expect that joint damage deferiorates and that repair is hardly ever seen. Although the results of the study in chapter 6 suggests that the gain in sensitivity due to reading chronologically outweighs the loss in specificity, it cannot be ignored that reading in the chronalogical order also mostllikely causes measurement error of the scoring method to be asymmetrical. In other words, the agreement between readers is likely to be better for negative scores (repair of damage) than for positive scores (deterioration of joint damage). This effect is further enlarged if the rule is applied that change-scores cannot become negative, as was done in the study of chapter 4. An asymmetrical distribution around zero of the measurement error of chronolagical joint damage scores causes problems when interpreting negative scores. Randomised clinical trials have shown that the newest drugs are able to stop deterioration of joint damage and moreover, in these trials a considerable number of negalive change-scores were also assessed. Should these negative scores merely be considered as part of the measurement error or are they evidence for some degree of repair? Individual case report, small series and supportive studies undertaken by the OMERACT study group on imaging suggest that repair of erosions in RA can occur. ${ }^{15,17}$ However, in order to be able to investigate whether the negative change-scares really reflects repair the measurement error should be distributed equally oround the zero difference line ${ }^{15}$; meaning that amount of agreement between readers is not reflected by the sign of the change-score. From this perspective, it thus seems preferable to conceal the sequence when reading films.

How the type of risk reduction can influence the required sample size in rondomised clinical trials.

In order to increase the contrast between an intervention and a control group, RCTs often include patients with a high prior risk on the outcome of interest by selecting on baseline predictors for that outcome. The general view is that such a strotegy limits the number of patients required, by increasing the statistical pawer. Chapter 9 discusses sample size calculations for future RA trials, based on a RCT in which radiographs were taken with a 3 -month interval. It is known that joint damage present at baseline is one of the strongest predictors for further progression of radialogical joint damage. Therefore it was expected that fewer patients would be needed for a RCT if patients were selected for the presence of joint damage at baseline. The results of the actual sample size calculations learned however that this expectation was false. To elucidate this counterintuitive observation, a literature search was done and showed that the way in which a treatment reduces the risk on an outcome (by an absolute (ARR) or by a relative risk reduction (RRR) model) influences the required sample sizes in trials with dichotomous outcome measures. In chapter 8 , the risk reduction 
madels are explained step-by-step and illustrated with hypothetical data. If a treatment mainly acts according to the RRR-model, selection of patients with a higher prior risk will result in a higher statistical power and thus smaller sample size. If a treatment acts mainly according the ARR model, selection of patients by prior risk may have divergent effects on the sample size required. Statistical power is then worst in case of a prior risk of on average $50 \%$, which can be the case when including a mixture of high, intermediate and low risk patients. As a consequence the required sample size will be higher. To explore the relationship between prior risk and treatment effect regarding DMARDS and their effect on radiological joint damage, the data of two RCTs were investigated. In both trials, the DMARD treatment group with the highest efficacy seemed to act mainly according to the ARR-model. Future research will have to reveal whether the DMARDs investigated indeed mainly act according to an ARR model and whether other DMARDS reduce radiological joint damage in a similar way.

\section{Detection of changes in radialogical joint damage within a 3 month interval}

The results of the study described in chapter 9 suggest that it is possible to reliably detect changes in joint damage due to RA already within three months. Changes in joint damage were seen by both the chronological and the paired Sharp/van der Heijde scoring method. Within these three months the reader did not detect repair of joint damage with the chronalogical method, although in this study it was allowed to also have negative change-scores for. To investigate whether the changes observed in the study would be large enough to be useful as an autcome measure in future (phase 1l) clinical trials of three months duration, sample sizes were estimated for imaginary trials. Acceptable sample size estimates were seen in several subsets of patients, depending on 1) how the investigated drug would reduce the individual risk on progiression of joint damage (by an absolute or relative risk reduction model), 2) on how damage was scored (with or without knowledge of the sequence of the films), 3) on the baseline risk of patients investigated and 4) whether 2 -sided or 1 -sided tests would be used. In several of these circumstances it was possible to detect an effect on structural damage. Consequence of these findings is that it might be possible to assess the optimal dose-and exposure range for slowing progression of radiological joint damage already in a very early stage of research.

MRI and ultrasonography (US) are also assumed to be able to detect changes within three months. The development of a scoring method for RA-related inflammation and joint damage on MRI is progressing and seems promising despite the complexity and amount of information produced. ${ }^{18.19}$ Especially the information on inflammation and surrounding soft tissue makes MRI an appealing method. The scoring system developed by the international MRIOMERACT group - the RAMRIS - does not include the joint in the feet until now. Recent results showed that also for MRI additional information of the inflammation and joint damage in the feet can be very useful. ${ }^{20}$ Funther, it should be noted that the image resolution in most clinically available MRI units appears to be insufficient far evaluation of the cartilage of the joints in the wrists and is thenefare not included in the scoring system." US is much less validated 
than MRI, but the available data suggest that for clinical practice US offers comparable information on both inflammatory and destructive changes in RA finger and toe joints. ${ }^{22}$ Some initials have been made to also develop a scoring system for the detection of RA-related inflammation and joint damage on US; however issues on reliability, standardization and documentation seem to limit its value in clinical trials. ${ }^{22}$ Despite that the RA-related findings by MRI seem very appealing and useful for the evaluation in clinical research, ane should keep in mind that the methodological issues discussed here should also be taken into account when evaluating the validity of the MRI for RA-related joint damage. Especially issues like sensitivity to change and the determination of the clinical relevance of (changes) in damage seen should be carefully investigated. Moreover, the long-term relationship of the "pathological conditions" on MRI and clinicall outcomes like functional capacity need to be determined yet. ${ }^{2.3}$ So the actual meaning of the abnormalities seen by MRI is not crystallized at this moment. Finally, besides the validity and reliability aspects of the scoring system

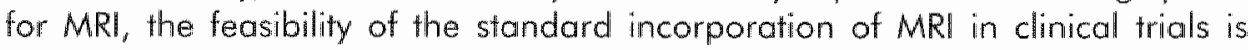
another issue. The use of MRI for the evaluation of clinical trials is still much more expensive and time consuming compared to the use of conventional radiographs.

At present radiographs remain the gold standard to assess structural damage. There are at least two well-validated scoring methods and more data become available that all underline the importance of radiagraphic damage. There is a clear relationship between functional capacity and other long-term outcomes. ${ }^{24}$ The data on the sensitivity to change with an interval as short as three months adds to the usefulness of this tool. The studies described in this thesis add further evidence to support the validity of both main scoring methods that are currently applied in research. 


\section{References}

1. Wassenberg s, Herborn G. Comparison of Larsen's and Sharp's method of scoring radiographs in Rhewmatoid arthritis. Arthritis Rhewn 1994;5upplement of 1994: $\$ 250$.

2. wan der Heijde $D$, Boonen $A$, Boers $M$, Kostense $P$, van Der Linden $S$. Reading radiographs in chronolagical order, in pairs or as single films has important implications for the discriminative power of rheumaloid atthitis clinical trials. Rheumatology (Oxford) 1999;38(12):1213-1220.

3. van der Heijde D, Dankent T, Nieman F, Rau R, Boers M. Reliability and sensitivity to change of a simplification of the Sharp/van der Helide radialogical assessment in theumatoid arthritis. Rheumatology (Oxford) 1999;38(10):941-947.

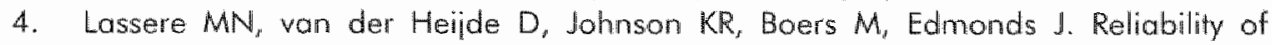
measures of disease activity and disease domage in rheumatoid arthritis: implications for smallest detectable difference, minimal clinically important difference, and analysis of treament effects in randomized controlled trials. J Rheumatol 2001;28(4):892-903.

5. van der Heijde D. Simon L, Smolen J, Strand V, Sharp J, Boers M, Breedveld F, Weisman M, Weinblatt $M$, Rau $R$, Lipsky P. How to report radiographic data in randomized clinical trials in theumatoid arthritis: guidelines from a roundtable discussion. Anthritis Rheum 2002;47(2):215-8.

6. Dans AL, Dans LF, Guyatt GH, Richardson S. Users" guides to the medical literature: XIV. How to decide on the applicability of clinical trial results to your patient. Evidence-Based Medicine Working Group. Jama 1998;279(7):545-9.

7. Guyatt GH, Juniper EF, Walter SD, Griffith LE, Goldstein RS. Interpreting treatment effects in randomised trials. BMJ 1998;316(7132):690-693.

8. Streiner DL, Norman GR Reliability. Heallth measurements scales. A practical guide to their development and use.: Oxford University Press, 1995:104-127.

9. Lassere MN, van der Heijde D, Johnson $K$, Bruynesteyn $K$, Molenaar E, Boonen A, Verhoeven $A_{x}$ Emery $P$. Boers $M$. Robustness and generalizability of smallest delectable difference in radiological progression. J Rheumatol $2001,28(4): 911-913$.

10. Guidance for Industry. Clinical development for drugs, devices and biological products for the treatment of Rheumatoid Athritis (RA). Rockville: Center for Drug Evaluation and Research, Food and Drug administration, 1997:1-56.

11. Boers $M$, Verhoeven AC, Markusse HM, vandeLarar M, Westhovens $R$, vanDenderen $J C_{x}$ ef al. Randomised comparison of combined step-down prednisolone, methotrexate and sulphasalazine with sulphasalazune alone in early rheumatoid anthritis. Lancet 1997;350:309-18.

12. Bathon JM, Martin RW, Fleischmann RM, Tesser JR, Schiff MH, Keystone EC, Genovese MC, Wasko MC, Moreland LW. Weaver AL, Markenson J, Finck BK. A comparison of etanercept and methotrexate in patients with earily rheumatoid anthritis. N Engl J Med 2000;343(22): 1586-93. 
13. Sharp JT, Strand V, Leung H, Hurley F, Loew-Friedrich 1. Treatment with leflunomide slows radiographic progression of rheumatoid arthritis: results from three pandomized cortrolled trials of leflunomide in patients with active rheumatoid arthritis. Leflunomide Rheumatoid Arthritis Investigators Group. Anthritis Rheum 2000;43(3):495-505.

14. Fries JF, Bloch DA, Sharp JT, MCShane DJ, Spitz P, Bluhm GB, Forrester D, Genani

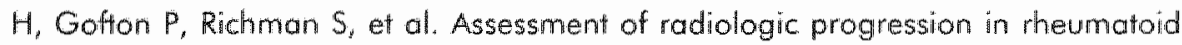
arthritis. A randomized, contralled trial. Arthritis Rheum 1986;29:1 -9.

15. wan der Heilde D, Landewé R. Imaging do erosion heal? Ann Rheum Dis $2003 ; 62$ (suppl.ily:iii 10.12 .

16. Lassere $M$, Bours $M_{r}$ wan der Heijde D, Boonen $A$, Edmonds J, Saudan A, Verhoeven AC. Smallest defectable difference in radiological progression. I Rheumatol $1999 ; 26(3): 731-739$.

17. Sharp JT, van der Heijde D, Boers $M$, Boonen A, Bruynesteyn $K$, Emery $P$, Genant $H K$, Herbom $G$, Jurik $A$, Lassere M, McQueen F, Ostergaard $M$, Peterfy $C$, Rau $R$, Strand V, Wassenberg S, Weissman B; Subcommittee on Healing of Erosions of the OMERACT Imaging Committee. Repair of erosions in rheumatoid arthritis does occur. Results from 2 studies by the OMERACT Subcommittee on Healing of Erosions. I Rheumatol 2003;30:1702-7.

18. Peterfy C, Edmonds J, Lassere M. Conaghan PG, Ostergaard M, McQueen FM, Genant $H_{4}$ Kllarlund $M$, Ejbjerg B, Stewart N, Bird P, Shnier R, OrConnor P, Emery $P$. OMERACT Rheumatoid Anthritis MRI Studies Module. J Rheumatol 2003;30(6):1364. 1365.

19. Ostergaard M, Peterfy $C$, Conaghan $P$, McQueen F, Bird P, Ejbjerg B, Shnier R, OConnor P, Klarlund M, Emery P, Genant $H$, Lassere M, Edmands J. OMERACT Rheumatoid Arthritis Magnetic Resonance Imaging Studies. Core set of MRI acquisitions, joint pathology definitions, and the OMERACT RA-MRI scoring system. I Rheumatol 2003;30(6):1385-6.

20. Boutry N, Larde A, Lapegue F, Solau-Gervais E, Flipo RM, Cotten A. Magnetic resonance imaging appearance of the hands and feet in patients with early rheumatoid arthritis. J Rheumatol 2003,30(4):671-9.

21. Ostergaard $M$, Szkudlarek $M$. Magnetic resonance imaging of soft tissue changes in rheumatoid arthritis wrist joints. Semin Musculoskelet Radiol 2001;5(3):257-74.

22. Ostergaard $M$, Szkudlarek $M$. lmaging in theumatoid arthritis--why MRI and ultrasonography can no longer be ignored. Scand I Rheumatol 2003;32(2):63-73.

23. Boers $M$. Value of magnetic resonance imaging in theumatoid arthritis? Lancet 2000;356(9240): 1458-1459.

24. van der Heijde D. Radiographic progression in theumatoid arthritis: does it reflect outcome? Does it reflect treatment? Anm Rheum Dis 2001;60 Suppll 3:iit47-50. 


\section{Chapter 11}

Summary

Samenvatting

Dankwoord

About the author

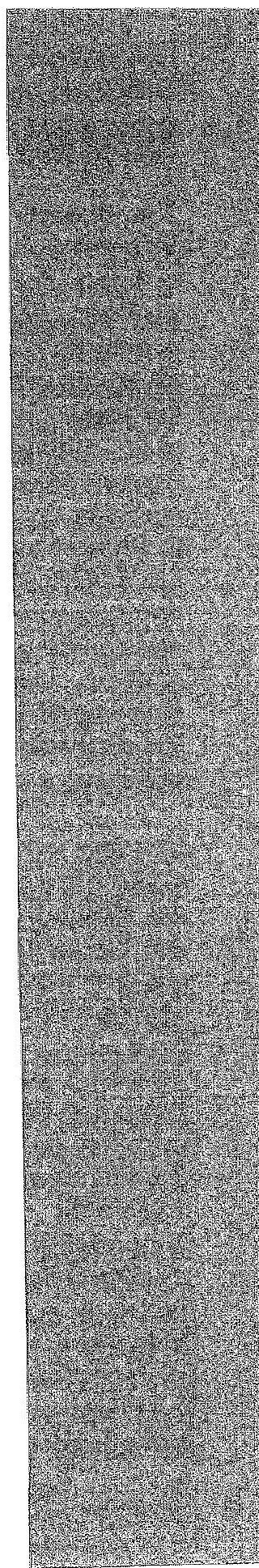




\section{Summary}

The disease rheumatoid arthritis (RA) is characterized by a chronic (sterile) inflammation of the joints, mainly localized in the smaller joints of the hands and feet. Persistent arthritis due to RA aften leads to permanent damage of the cartilage and underlying bone. The damage can be visualized on plain radiographs. Anti-inflammatory drug can inhibit the inflammation and may slow down or prevent deterioration or occurrence of joint damage. In order to investigate whether these drugs indeed are able to moderate joint damage, plain radiographs are included in clinical research. Further, to be able to analyse the efficacy of the drug under investigation, the amount of damage on cartilage and bone needs to be quantified. Quantification of joint damage due to RA is called "scoring" and the standardized approach of scoring pre-selected joints is called a "scoring method". How well the ability of a drug to modify joint damage can be investigated mainly depends on the validity, reproducibility and responsiveness of the scoring method used. Several radialogical scoring methods have been described and evaluated in the past. The methods developed by Sharp and Larsen and their modifications - like the Sharp/van der Heijde method and the Larsen/Scolt method-are the most widely used methods at this moment.

Chapter 2-9 of this thesis describe research performed to solve several outstanding issues regarding scoring joint damage by means of the Sharp/van der Heiide method and the Larsen/Scott scoring method. This with the goal to use the optimal radiological scoring method in future clinical research on RA and consequently to be able to answer research questions with less patients and/or less money.

Chapter 2 describes how the discriminative capacity and responsiveness of the Sharp/van der Heijde and the Larsen/Scolt relate to each other. For this purpose so-called generalizability analyses were used. The analyses showed that the discriminative capacity and responsiveness of the methods were both highly acceptable and comparable if mean group scores were calculated and compared. If the percentage of patients that actually showed deterioration were calculated and compared, the Sharp/van der Heijde scores were measured with more precision.

With these andyses also the issue of the optimal number of investigators (scorers) used in clinical research to score joint damage could be addressed. The analyses showed that the discriminative capacity and responsiveness of both methods changed most if two instead of one scorer were used.

Prevention of erosions (destruction of the bony surface underlying the cartilage) in undamaged joints is often considered more important than prevention of 
deterioration in already damaged joints. Chapter 3 however showed that important information on the efficacy of the drug investigated might be missed and/or more patients are needed if the investigation is limited to undamaged joints. It thus seems preferable to judge both undamaged as well as already damaged jaints in clinical research.

In chapter 4 the so-called minimal clinically important difference (MCID) was assessed for both scoring methods. An international expert panel was used to define this MCID. The panel consisted of five experienced rheumatologists who are used to judge radiographs of patients themselves but who were not trained to quantify joint damage in a standardized way. The rheumatologists judged independently sets of radiographs that had been obtained with 1 -year intervals. They were asked to state whether they saw progression of joint damage or not. If they stated that they noticed progression of damage they were further asked whether they judged that amount of progression of that amount that they wanted to change the patient's therapy. Or in other words, whether they found the amount of progression clinically relevant. The panel members were asked to judge the progression noticed for four different types of patients; patients with 1) recently diagnosed $R A$ with mild symptoms, 2) recently diagnosed $R A$ with severe symptoms, 3) advanced RA with mild symptoms and 4) advanced RA with severe complaints. The panel's judgments were compared with the average changes in joint damages measured by the Sharp/van der Heijde and the Larsen/Scott method. These scores were independently obtained by two experienced scorers for each scoring method.

The number of patients that actually show clinically important deterioration (or repair) of joint damage is often calculated because it can give additional information on the efficacy of the drug investigated. If one wants to calculate the number of patients that show clinically important deterioration (or repair) of joint damage one needs to know the MCID. In the past, the sa-called smallest detectable difference has - as alternative- been used as cutoff value to express the continuous scores as an outcome with two answer possibilities (progression yes or no) [the smallest detectable difference is the difference between two scores that is greater than the measurement error of the scoring method. This statistical method assesses the measurement error of the scoring method that occurs while repeating the scoring]. In chapter 4, the MCID was therefore compared with this smallest detectable difference.

The results in chapter 4 showed that the panel on average judged a change in joint damage in the order of the magnitude of the smallest detectable difference of the Sharp/van der Heijde method as clinically relevant. The difference between the MCID and the smallest detectable difference of the Larsen/Scott method was remarkable: the panel judged changes smaller than the measurement error of the Larsen/Scott method already as clinically relevant. Additional analyses showed that this probably can be explained by the fact that the panel also took the narrowing of joint spaces (due to cartilage loss) into account. If one quantifies joint damage with the Larsen/5cott method, lasses of cartilage are hardly taken into account in the scores. 
In order to validate the judgments of the panel, the radiographs were also judged by a couple of radiologists who were highly experienced in judging joint damage due to RA. The results described in chapter 5, showed that the radiologists were more reserved in judging changes as important than the panel of rheumatologists. The panel of rheumatologists were inclined to change therapy in cases of progression of joint damage that the radiologists reported as not substantial. The radiologists further judged less sets of radiographs as progressive. This study confirmed former research that showed that the profession of the panel members can strongly influence panel results. The panel used in the study of chapter 4 could however not include radiologists. After all, this panel had to judge whether they wanted to change the treatment strategy of a patient based on the deterioration of joint damage. Radiologists are not trained in making inferences for patients' treatment strategies. In conclusion, if possible, it seems preferable to include clinicians of different profession when using an expert panel in your research, in order to be able to generalize the results as much as possible.

Research has shown that significant more progression is detected if the scorer knows the chronological order of the radiagraphs he/she has to judge. Explanation for this is most likely that one is able to correct maximally for variation on quality of the radiographs or variation in position of the hands and/or feet if the correct order is known. However, question remained whether knowledge regarding the carrect arder of the radiographs not primarily leads to overestimation of progression instead of a more precise detection. In order to answer this question, the influence of knowledge of the chronological arder on the detection of clinical relevant progression of joint damage by the scoring methods was investigated in chapter 6 . The clinical relevance for recent diagnosed RA patients with severe symptoms was used for this purpose and was defined by the same panel as used in chapter 4 . The results showed that the scoring methods were more sensitive in detecting clinical relevant progressions if the scorers knew the correct order of the radiographs. The results showed however also that the gain in sensitivity lead to some not ignorable loss in specificity, resulting in the wrangly classification of progression of joint damage as clinical relevant in some cases.

Besides that knowledge of the chronological order leads to more sensitive scoring methods, this knowledge on the other hand also appears to make the interpretation of repair of damage difficult. Recent research has suggested that joint damage due to RA is not that permanent as assumed. In order to be able to investigate this, scorings methods should be used in which the agreement between the positive change scores (progression) is equal to the agreement between the negative change scores (repair of damage). Because this is not the fact if the chronological order is known to the scorers, this method seems not to be recommended for research at this moment.

In the course of the above described research, it became clear that the formulas used to calculate the smallest detectable difference should be adapted if 
radiographs are scored in pairs, as is usual for the judgment of joint damage due to RA. With paired scores, sets of radiographs of one patient are judged simultaneously, causing the measurement error of the different scores of that patient to be related. The correct formulas to calculate the smallest detectable difference if radiographs are judged in pairs is presented in chapter 7 lcalled the smallest detectable change). This chapter further showed that with the original smallest detectable difference, the measurement error of detecting a change in scores is overestimated if radiographs are scored in pairs.

Research of new treatments often includes patients with a high risk at the disease outcome to be prevented/diminished (like joint damage). This is done with the aim to make the contrast in the number of patients with the eventual outcome to be prevented as large as possible between the patients in the treatment and the control group. The presence of joint damage at the start of a clinical study is one of the best predictors for progression of joint damage during the trial. Therefore, it was expected that less patients would be needed to answer a research question when patients with much joint damage would be selected for inclusion. Chapter 8 describes why this is not always true for outcomes with two answer possibilities. In summary, the effect of a selective inclusion of patients with a high risk depends on how the treatment reduces the risk on the outcome investigated. Not in all cases this is leading to the expected enhanced effect.

Chapter 9 presents the results of a study that investigated whether it is possible to detect change in joint damage on plain radiographs taken with a 3 -month interval. In studies testing a new drug, the modifying effect of the new treatment on joint damage is usually investigated after 12 months. Recent research has however shown that also after six months significant differences in progression of joint damage can be found. The clinical studies that evaluate the most optimal dosage and treatment duration, so-called phase 2 studies, however usually last three months. Therefore it is interesting to investigate whether it is possible to detect progression of joint damage already in a 3-month interval. Chapter 9 showed that the Sharp/van der Heijde method was able to detect change in joint damage on plain radiographs taken with a 3-month interval, with or without known chronological order of the radiographs. Additional analyses further showed that also with a 3-month interval, the number of patients needed to detect significant differences between a treatment and control group could be acceptable when plain radiographs are taken with a 3 -month interval. 


\section{Samenvatting}

De ziekte Reumatoide Artritis (RA) wordt gekarakteriseerd door een chronische (steriele) ontsteking van de gewrichten die zich met name manifesteert in de gewrichten van de handen en de voeten. De chronische gewrichtsontsteking bii RA kan leiden tot onherstelbare schade van het kraakbeen en (of) het daaronder liggend bot. Deze beschadigingen kunnen worden gezien op röntgenfoto's. Met geneesmiddelen kan de ontsteking in de gewrichten geremd warden waardoor de (toename) van de gewrichtsschade (gedeeltelijk) voorkomen of verminderd kan worden. Om tijdens klinisch wetenschappelijk onderzoek te kunnen beoordelen of een geneesmiddel deze modificerende werking heeft worden er röntgenfoto's gemaakt. De mate van schade aan het bot en kraakbeen op de foto's moet in getal uitgedrukt worden om de werking van verschillende geneesmiddelen daadwerkelijk te kunnen beoordelen en/of vergelijken. Het toekennen van een waarcle aan de schade wordt 'scoren' genoemd en het op gestandaardiseerde wilze scoren van tevoren geselecteerde gewrichten wordf scoren volgens een bepaalde 'scoringsmethode' genoemd. Hoe goed een geneesmiddel kan worden beoordeeld hangt in belangrijke mate af van hoe betrouwbaar de scoringsmethode is. Dit betreft de reproduceerbaarheid (onder dezelfde omstandigheden dezelfde score geven) en gevoeligheid voor verandering van de gebruikte scoringsmethode. Verscheidene scoringsmethodes zilin in het verleden beschreven en geëvalueerd. De heden meest gebruikte methodes zijn de door Sharp en de door Larsen ontwikkelde of daarvan afgeleide methodes (zoals de zogenaamde Sharp/van der Heijde en de Larsen/Scot methode).

In dit proefschriff wordt in hoofdstuk 2-9 onderzoek beschreven naar een aantal nog openstaande vragen bil het scoren van gewrichtsschade met behulp van de Sharp/van der Heijde en Larsen/Scott scoringsmethode. Dit met als doel om in toekomstig wetenschappelijk onderzoek de optimale scoringsmethode te kunnen gebruiken waardoor mel minder patiënten en minder geld een betrouwbaar antwoord kan worden verkregen.

Hoofdstuk 2 beschrift hoe het onderscheidend vermogen en de gevoeligheid voor verandering van de Sharp/van der Heijde en de Larsen/Scott methode zich ten opzichte van elkaar verhouden. Dit is anderzocht met zogenaamde 'geneneraliseerbaarheidanalyses'. Deze analyses lieten zien dat het onderscheidend vermogen en de gevoeligheid voor verandering voor beide methodes acceptabel tot goed en vergelijkbaar waren indien gemiddelde scores van groepen met elkaar worden vergeleken in een onderzoek. Indien echter in een onderzoek de percentages patiënten dat werkelijk toename (of afname) van schade laten zien worden vergeleken dan lijken de scores van de Sharp/van der Heijde methode met grotere precisie te worden gemeten. 
Mel behulp wan deze analyses kon ook een uitspraak worden gedaan over het optimale aantal te gebruiken beaordelaars bij klinisch wetenschappelik onderzoek. Het onderscheidend vermogen en de gevoeligheid voor verandering van beide scoringsmethodes bleek het meest te verbeteren indien twee in plaats van éen beoordelaar werden gebruikt.

Vaak wordt in onderzoek het voorkómen wan erosies (beschadiging van het oppervlak van het bot aangrenzend aan het kraakbeenj in miet beschadigde gewrichten belangrijker geacht dan het voorkómen van progressie wan de schade van gewrichten die reeds erosies hebben. Hoofidstuk 3 laat echter met behulp van gegevens vam een recent geneesmiddelenonderzoek zien dat belangrijke informatie over het geneesmiddel kan worden gemist en/of meer patiënten nodig ziin indien alleen de onbeschadigde gewrichten worden beoordeeld Het lijkt dus raadzaam om in wetenschappelijk onderzoek de gewrichtsschade in zowel de onbeschadigde als in de reeds beschadigde gewrichten te beoordelen, zodat mogelijk met minder patiènten een antwoord kan worden verkregen op de vraagstellingen.

In hoofdstuk 4 is voor beide scoringsmethodes hel kleinste klinisch relevante verschil in gewrichtsschade geschat. Hiervoor is gebruik gemaakt van een international expertpanel. Dit panel bestond vit viff ervaren reumatolagen die in de praktiik zelf hun röntgenfoto's beoordelen maar niet getraind waren on op een gestandaardiseende wijze een getal aan de schade te geven. De reumatologen werden gevraagd om onathankelijk van elkaar fotosels met én jaar tussentijd te beaordelen op het wel of niet ontstaan en/of toenemen wan de gewrichtsschade. Indien ze progressie van de gewrichtsschade zagen, moesten ze aangeven of ze de verandering van dusdanige aard vonden dat ze de therapie van de patiënt wensten aan te passen. Met andere woorden, aangeven of de progressie wel of niet gezien moest worden als klinisch relevant. Dit werd gevraagd voor vier verschillende typen patiënten; patiënten met 1) recent vastgestelde $R A$ en milde klachten, 2) recent vastgestelde $R A$ en ernstige klachten, 3 ) langer bestaande RA en milde klachten en 4 ) langer bestaande RA en ernstige klachten. Het oordeel van het panel werd vervolgens vergeleken met de gemiddelde Sharp/van der Heijde en Larsen/Scott pragressiescores thet verschil tussen de score bij het begin en ến jaar later' vam wee beoordelaars. Hiervoor werden per scoringsmethode de röntgentoto's onafhankelikk gescoord door twee verschillende ervaren beoordelaars.

Het aantal patiënten dat daadwerkelik een klinisch relevante verandering laat zien, kan interessante aanvullende informatie opleveren bij het analyseren vam klinisch wetenschappelijk onderzoek. Om dit te kunnen doen moet men echter weten wat het kleinste klinisch relevante verschil is in het verleden is als altematief vaak het zagenaamde kleinst meetbare verschil gebruikt als akkappunt om de continue scores van de scaringsmethodes vit te drukken ais een maat met hwee vitkomsten (wel of geen progressie) [het kleinst meetbare verschil is een verschil dat groter is dan de meetfout van de scoringsmethade. Deze statistische mat schat de meetfout wan die scoringsmethode die optreedt 
tijdens het reproduceren van scores]. In hoofdstuk 4 is dientengevolge het door het panel bepaalde kleinst klinisch relevante verschil vergeleken met het kleinst meetbare verschil.

Hoofdstuk 4 liet zien dat het panel gemiddeld gezien schade ter grootte van het kleinst meetbare verschil wan de Sharp/van der Heilide scoringsmethode reeds als klinisch relevant beoordeelde. Het verschil fussen het kleinst meetbare verschil van de Larsen/Scott methode en het kleinste klinisch relevante verschil was echter opmerkelijk. Bij progressie kleiner dan de meeffout van de Larsen/Scott methode oordeelde het panel dat ze de therapie all wilde wijzigen. Aanvullende analyses lieten zien dat dit waarschijnlijk grotendeels verklaard kan worden door het feit dat het panel ook in belangriike mate de gewrichtsspleetvernauwing (een gevolg van kraakbeenverlies) in hun oordeel meenam. De gewrichtsspleetvernauwing wordt bij de Larsen/Scott scoringsmethode slechts in geringe mate meegenomen bif het scoren van de gewrichtsschade.

Ter validatie van het in hoofdstuk 4 gebruikte expertpanel, werden de röntgenfoto's ook voorgelegd aan twee radiologen met vitgebreide ervaring in het beoordelen van gewrichtsschade tengevolge van RA. De resultaten in hoofdstuk 5 lieten zien dat de radiologen meer gereserveerd waren in hun oordeel dan hef panel reumatologen. Het panel reumatologen gaf aan de therapie te willen aanpassen bij veranderingen in radiologische schade die door de radiologen niet als substantieel beoordeeld werden. Bovendien beoordeelde de radiologen in totaal minder fotosets als progressief. Deze studie bevestigt de in het verleden vitgevoerde anderzoeken die lieten zien dat het beroep van een panellid de uitslagen van een panel sterk kunnen beoordelen. Bij het experiment vitgevoerd in hoofdstuk 4 was het echter niet mogelijk om ook radiologen te includeren Dit panel moest immers beoordelen of ze de therapie van de patiënten wilde wijzigen en radiologen zijn niet getraind in hef doen van uitspraken over het therapeutische beleid van een patiënt. Samenvattend lijkt het dus echter wel raadzaam om leden van verschillende beroepen te laten deel nemen in een expertpanel indien dit mogeliik is. Dit met het oog op een zo groot mogelijke generaliseerbaarheid van de resultaten

Uit onderzoek is gebleken dat indien de chronologische volgorde van een serie röntgenfoto's bekend is bii de beoordelaar(s) er significant meer progressie gemeten wordt dan wanneer onbekend is welke foto de eerste in de tijd is. Een logische verklaring voor deze bevinding is dat indien de volgorde van röntgenfoto's bekend is maximaal gecorrigeerd kan worden voor variatie in kwaliteit of in de positie van de handen/voeten tussen de foto's. De vraag bleef echter of de kennis over de correcte volgorde van röntgenfoto's niet juist leidt tot een overschatting in plaats tot eem preciezere schatting. Om hierover een uitspraak te kunnen doen, is in hoofdstuk 6 de invloed van het al dan niet bekend zijn van de chronologische volgorde van een serie röntgenfoto's op het detecteren van klinisch relevante progressie door de scoringsmethodes onderzocht. Hiervoor is gebruik gemaakt van het oordeel van hetzelfide 
expertpanel als beschreven in hoofdstuk 4 voor de groep RA patienten met ernstige klachten en waarbii recent de diagnoses RA is gesteld. De resultaten lieten zien dat de scoringsmethodes veel gevoeliger waren voor het oppikken van patiënten met klinisch relevante progressie indien de volgorde van de röntgentoto"s bij de beoordelaars bekend was. Dit ging echter wel ten koste van enige specificiteit van de methodes, resulterend in het soms ten onrechte classificeren van de progressie als klinisch relevant.

Naast het feit dat scoren met kennis over de volgorde van de röntgenfolo's blijkt te leiden tot meer gevoelige scoringsmethodes, blijkt het geven van deze kennis echter ook de interpretatie van herstel van schade te bemoeilijken. Recent onderzoek suggereert dat de gewrichtsschade bij RA toch niet zo definitief is als men gedacht heeft. Om dit beter te kunnen onderzoeken, heeft men scoringsmethodes nodig waarbij de overeenstemming tussen de beoordelaars bij zowel progressie als bii herstel van de gewrichtsschade gelijk is. Daar dit niet het geval is indien de chronologische volgorde bekend is bij de beoordelaar(s), lijkt deze methade momenteel niet aan te raden voor het doen van wetenschappelijk onderzoek.

Gedurende bovenstaand onderzoek werd duidelijk dat de formules gebruikt om het kleinst meetbare verschil te bepalen aangepast moeten worden indien men dit toepast op gepaarde waarnemingen, zoals gebruikeliik bii het beoordelen van de gewrichtsschade bij RA. Met gepaarde waarnemingen word hier het tegelijk beoordelen van opeenvalgende röntgenfoto's van één patiënt bedoeld, waardoor de meetfout van de verschillende scores van één patiënt met elkaar samenhangt. In hoofdstuk 7 wordt de juiste formule gepresenteerd om het kleinst meetbare verschil te bepalen indien de scores 'gepaarde' waarnemingen zijn. Tevens laat dit hoofdstuk zien dat met de originele formules de meetfout wordt overschat indien scores 'gepaard' zijn bepaald.

Bii onderzoek naar een nieuwe behandeling warden vaak patiënten geincludeerd die een hoag risico hebben op de door de nieuwe behandeling te voorkomen/verminderen van de ziektemaat (zoals gewrichtsschade). Dit wordt gedaan om het te verwachten verschil in patiënten tussen de behandelgroep en de controlegroep zo groot mogelijk te maken. Hierdoor kan met minder patiënten reeds een goed oordeel worden geveld over de nieuwe behandeling. Daar hel hebben van gewrichtsschade éen van de sterkste voorspellers is voor het ontstaan van nieuwe gewrichtsschade, zou men verwachten dat bii selectie van patiënten met veel gewrichtsschade met minder patiënten een verschil tussen de behandelgroepen kan worden aangetoond. In hoofdstuk 8 wordt beschreven waarom dit bii vitkomstmaten met twee antwoordmogelijkheden (zoals wel of geen progressie van gewrichtsschade) echter niet altijd opgaat. Samenvattend blijkt het effect van selecteren van een 'hoog risicogroep' af te hangen van de wijze waarop een behandeling de kans op de te onderzoeken uitkomstmaat vermindert. Niet in alle gevallen levert dit het verwachte gunstige effect. 
In hoofdstuk 9 worden de resultaten beschreven van een studie die onderzocht of met röntgenfoto's ook veranderingen in gewrichtsschade kunnen worden aangetoond in een periode van drie maanden. Volgens de richtlijnen van de Amerikaanse FDA (Food \& Drug Association) wordt in de meeste onderzoeken naar nieuwe geneesmiddelen voor RA pas na 12 maanden geëvalueerd of het nieuwe middel een modificerende werking heeft op de gewrichtsschade. Recent geneesmiddelenonderzoek heeft echter aangetoond dat ook na zes maanden reeds verschillen in gewrichtsschade kon worden gezien. Daar de klinisch wetenschappelijk studies die de optimale dosis en behandelingsduur van een geneesmiddel onderzoeken, de zogenaamde fase 2 studies, meestal drie maanden duren, is het dus interessant om te onderzoeken of je met röntgenfoto's reeds na drie maanden verschillen in groepen kan detecteren. Het onderzoek beschreven in hoofdstuk 9, liet zien dat de Sharp/van der Heilde methode significante veranderingen kon detecteren met een tussentijd van drie maanden. Aanvullende analyses lieten bovendien zien dat ook bij een interval van drie maanden, onder de juiste omstandigheden, het aantal patiënten dat nodig zal zijn om verschillen tussen een behandel- en controlegroep aan the tonen acceptabel is. 


\section{Dankwoord}

Vele personen hebben direct en indirect bijgedragen aan de totstandkoming van dit proefschrift en bovendien het promoveren vaak minder eenzaam gemaakt dan welke het meestal is. Graag wil ik van de gelegenheid gebruik maken om hen allen te bedanken.

Ten eerste wil ik alle patiënten bedanken die door hun deelname aan de verschillende klinische onderzoeken er voor hebben gezorgd dat er voldoende röntgenfoto's beschikbaar waren voor het in dit proefschrift gepresenteerde methodologisch onderzoek. Geen van allen heb ik mogen ontmoeten, maar zonder hun bereidheid om deel te nemen aan klinisch onderzoek was dit proefschriff nooit tot stand gekomen.

Miin promotoren, prof.dr. Désirée van der Heijde en prof. dr. Sjef van der Linden bedank ik voor het in mil gestelde vertrouwen om dit onderzoek vit te voeren en vorm te geven. Jullie hebben me een kans geboden waar ik niet lang over na heb hoeven denken. Désirée, jouw grote interesse voor onderzoek naar vitkomstmaten bij aandoeningen van het bewegingsapparaat heeft me doen kennis maken met een intrigerend deel van wetenschap. Tevens heeft jouw inzet en internationale bekendheid me de unieke mogelijkheid gegeven om te mogen ruiken aan het internationale spel van de wetenschap. Voor dit alles ben ik je zeer erkentelijk. Sjef, van een afstand, maar toch betrokken heb je meegewerkt aan het realiseren van dit proefschrift. Speciaal bedankt voor je kritische opmerkingen over de opzet van het onderzoek en de uiteindeliike manuscripten. Deze ziin er viteraard altijd beter van geworden.

Het Reumafonds dank ik voar de financiering van het in dit proefschrift beschreven onderzoek en mijn aanstelling ais A.I.O.

Leden van de beoordelingscommissie, prof. dr. van Schayck, prof. dr. Boers, prof. dr. van Engelshoven en prof. dr. van der Vleuten, harteliik dank voor het beoordelen van mijn proefschrift en jullie bereidheid aan de oppositie deel te nemen. Dear prof. dr. Sharp, thank you for reviewing my thesis.

Naast prof. van der Heijde en prof. van der Linden heb ik tijdens verschillende fases van mijn onderzoek constructieve adviezen en hulp mogen ontvangen van verscheidene anderen. Met name wil ik bedanken prof. dr. Maarten Boers. Maarten, je bent bij vele onderdelen van miin onderzoek persoonlijk en actief betrokken geweest. Ondanks dat je al weer een ruime tijd vertrokken bent uit Maastricht en zeer druk bent met andere projecten heb je altijd interesse getoond en een bijdrage willen leveren aan het onderzoek en de manuscripten. Prof. dr. Cees van der Vleuten, wil ik bedanken voor zijn bijdrage aan het 
onderzoek en manuscript beschreven in hoofdstuk 1. Cees, jouw expertise en nimmer aflatende bereidheid om mee te denken als ik weer eens s'nachts iets. had bedacht, heb ik altijd zeer gewaardeerd. Maarten en Cees, bedankt voor de samenwerking en ik vind het een eer dat jullie in miln beoordelingscommissie hebben willen plaats nemen.

Dr. Robert Landewé wil ik hier tevens met name noemen. Robert, ondanks dat je geen officielle functie hebt gehad bij het tot stand komen van mijn proefschrift heeft jouw hulp en expertise tegen het eind toch regelmatig de voortgang van miin onderzoek en manuscripten bespoedigd op momenten dat anderen daar minder tijd voor vrij konden maken. Nog hartelijk bedankt hiervoor.

Bij deze wil ook statisticus dr. Piet Kostense bedanken voor zijn essentiële bijdrage aan hoofdstuk 8 . Piet, jouw bereidheid om open naar mijn - nief altijd statistisch juist verwoorde - ideeën te luisteren heeft uiteindelijk het licht op groen gezet voor dit hoofdstuk. Dank hiervoor.

Hereby I want to especially thank the rheumatologists and radiologists who judged or scored the radiographs for the studiesand all other co-authors who gave valuable input for the articles: dr. A. Boonen, prof. dr. B Breshnihan, prof. dr. J Edmonds, dr. B Griffiths, dr. F Gubler, dr. H Houben, dr. M. Lassere, prof. H Paulus, prof. P Peloso, dr. A. Saudan, dr. A. Verhoeven and dr. R Weijers. Your expertise and cooperation was of great value.

Een prominente plaats in mijn dankwoord verdient tevens mijn goede excollega, vriendin en oud kamergenoot Astrid Wanders. Astrid, onze vele discussies heeft mii altijd veel plezier gebracht en heeft bovendien geleid tot onze samenwerking in hoofdstuk 7 . Hopelijk zullen ze ook buiten het reumatologisch onderzoek woortgezet worden. Als vriendin en als collega kan je als voorbeeld dienen voor velen. Dank voor alles en ik vind het reuze fijn dat je mijn paranimf wilt zijn!

Ook mijn andere voormalige kamergenoten, medeonderzoekers en collega's bii de reumatologie wil ik viteraard zeer bedanken voor hun meedenken en meeleven. Met name wil ik speciaal noemen: Jolanda Braver, Simone Gorter, Claudia de Haan, Liesbeth Heuft, Erik de Klerk, Thea Schoonbrood en Guy Schulpen. Jullie interesse en hulp heeft me altiid zeer gesteund.

Tevens wil ik mijn nieuwe collega's bij Mapi Values bij deze bedanken voor hun getoonde interesse en het creëren van een omgeving waarin het mogelijk is om een proefschrift af te ronden naast het hebben van een drukke baan.

Femke Hoekstra-Drost en Tiny Wouters, wil ik speciad bedanken voor hun hulp bij het klaar maken van dit boekje. Tiny, je enthousiasme was als een warm bad, dank hiervoor. 
Verder wil ik alle wrienden en familie bedanken voor hun belangstelling en steun voor mijn onderzoek, ondanks dat het niet altijd te volgen was waar ik nu precies mee bezig was.

Tot slat wil ik mijn ouders, mijn broer (en paranimf) Raymond en Gerbrand bedanken voor hun nimmer aflatende steun.

Mam en $\mathrm{Pa}$, jullie onvoorwaardelijke liefde woor mij en respect voor een ieders keuzes heeft gemaakt dat ik onbevangen elke nieuwe uifdaging aan kan gaan. Ilk ben jullie hiervoor zeer dankbaar.

Raymond, niemand heeft een groter vertrouwen in mijn kunnen dan jii. Je beseft waarschijnlijk niet hoeveel dal altijd voor me betekend heeft. Dank!

Gerbrand, wat kan ik meer zeggen dan dat onze liefde me in alle fases van ons leven altiid alle vertrouwen in de toekomst heeft gegeven. 


\section{About the author}

Karin Bruynesteyn was born on January $26^{\text {th }}, 1972$ in Haarlem. She graduated from secondary school in 1990, and started studying Medicine at the Free University of Amsterdam. She graduated in medicine in 1994 and became a doctor of medicine in 1997 (cum laude). In 1998 she worked as a house officer ("AGNIO") in the Internal Medicine ward of the Flevo Hospital in Almere. In 1999 she became a PhD student at the division of Rheumatology of the University of Maastricht and the research institute ExTra in Maastricht. The Dutch Arthritis Association financed this PhD project. During her PhD project, she followed the postgraduate training in epidemiology at the Free University of Amsterdam and passed the theoretical examinations in 2002. From July to November 2002 she worked as a freelance worker for AMTEC B.V. (a research agency of the University Hospital Maastricht) for which she scored radiographs of clinical trial patients. For the article presented in chapter 3 , she was rewarded with the "Impact Factor of the year 2003" award from the "niernal Department of the University Hospital Maastricht. Since 2003, Karin Bruynesteyn has been employed at Mapi Values the Netherlands B.V. Mapi Values is an international consultancy that specializes in health economics research and the development and evaluation of patient-reported outcome measures. 